\title{
Numerical Criterion for the Stabilization of Steady States of the Navier-Stokes Equations
}

\author{
Chongsheng CaO, IOANnis G. KeVReKidis, \\ AND EDRISS S. TITI
}

Dedicated to Professors Ciprian Foias and Roger Temam

ABSTRACT. This paper introduces an explicit numerical criterion for the stabilization of steady state solutions of the NavierStokes equations (NSE) with linear feedback control. Given a linear feedback controller that stabilizes a steady state solution to the closed-loop standard Galerkin (or nonlinear Galerkin) NSE discretization, it is shown that, if the number of modes involved in the computation is large enough, this controller stabilizes a nearby steady state of the closed-loop NSE. We provide an explicit estimate, in terms of the physical parameters, for the number of modes required in order for this criterion to hold. Moreover, we provide an estimate for the distance between the stabilized numerical steady state and the actually stabilized steady state of the closed-loop Navier-Stokes equations. More accurate approximation procedures, based on the concept of postprocessing the Galerkin results, are also presented. All the criterion conditions are imposed on the computed numerical solution, and no a priori knowledge is required about the steady state solution of the full PDE. This criterion holds for a large class of unbounded linear feedback operators and can be slightly modified to include certain nonlinear parabolic systems such as reaction-diffusion systems. 


\section{INTRODUCTION}

There is, recently, a reinforced interest in the important subject of flow control (see for example the monograph [36] or the proceedings of the recent NSF workshop on Control of Flows [56]). This is certainly due to an array of emerging technologies, such as the microfabrication of minute, programmable (and cheap) sensors and actuators. Part of the excitement is certainly due to cross-fertilization at the intersection between physical fluid mechanics and nonlinear partial differential equations. Indeed, the last few years have seen extensive applications of systems theory approaches and methods to the control and stabilization of flow models (see for example $[1,9,13,14,41,55]$ ).

For physical problems modeled by partial differential equations (as fluid flow is governed by the Navier-Stokes Equations (NSE), ) the state in the corresponding control problem is infinite dimensional. Practical considerations (finite precision, memory capacity of computers that would implement control action) dictate the discretization of the distributed parameter system (DPS) to a finite dimensional dynamical system $([3,22,68])$. How well this (lumped) discretized system captures the dynamics of the original PDE is the subject of ongoing research (see, e.g., $[42,49,50,66,74]$ and references therein). The dissipative nature of many evolutionary PDEs forms the basis of arguments for the qualitative validity of such approximations.

Using existing control methodologies, it is conceptually straightforward to synthesize finite-dimensional controllers for the finite dynamical systems resulting from such (finite difference, finite element, spectral) discretizations. A definitive sequence of articles by Balas, Curtain, Sakawa and others over the last 20 years $([3,4,5,6,7,22,68])$ states that such finite dimensional controllers can be used to stabilize linear infinite dimensional systems from which the discretization originates. The controllers should of course be based on a sufficiently converged discretization of the PDE (see [5] and also later [7]). One of our goals in this article is to prove that such finite dimensional controllers can indeed stabilize unstable steady states of the closed-loop NSE, and provide estimates of the necessary discretization dimension.

High order and ill-conditioning, however, make the routine design of controllers for such problems a difficult issue on its own ([71]); recourse to nontrivial computational methods is required in order to assess basic properties of the linearized state space models, such as controllability, stabilizability, observability and stability $([10,25])$. A second level of model reduction then becomes necessary: after the reduction of the infinite-dimensional system to a ("large") finite dimensional one, we seek to exploit the dissipativity of the original PDE to construct (or approximate) accurate, dynamic, "small" finite dimensional models that can be used in controller design. Such "further reduced" models are often based on modal representations of the dynamics, the modes coming from the leading part of the linearized problem ([8]), from their Krylov subspace approximations ([44]), from empirically determined eigenfunctions ([43], and references therein), or from an 
appropriate (dissipative) part of the linear problem operator, such as the eigenfunctions of the Stokes operator in the case of the Navier-Stokes equations ([17], [73], [74]). Beyond the qualitative similarity with singular perturbation methods that construct invariant manifolds and exploit them in controller design for finite dimensional systems ([57]), there is an extensive interest in the use of inertial manifolds and approximate inertial manifolds for closed loop dynamics analysis and controller design (see, for example, [12, 15, 69, 70]). Motivated by the theory of Inertial Manifolds and Approximate Inertial Manifolds, we will also explore a second (Nonlinear Galerkin, NLG) model reduction step.

In this paper we demonstrate our results and tools on a specific system, the Navier-Stokes equations of viscous incompressible fluids. We use the NSE as an illustrative paradigm. However, our results and technique hold for a larger class of nonlinear dissipative evolution systems of equations.

The Navier-Stokes equations

$$
\begin{array}{ll}
\frac{\partial u}{\partial t}-v \Delta u+(u \cdot \nabla) u+\nabla p=g, & \text { in } \Omega, \\
\nabla \cdot u=0, & \text { in } \Omega, \\
u=0, & \text { on } \partial \Omega, \\
u(x, 0)=u_{\text {in }}(x) &
\end{array}
$$

are the equations that govern the motion of viscous incompressible flows in a domain $\Omega$, of $\mathbb{R}^{2}$ or $\mathbb{R}^{3}$, subject to the no-slip Dirichlet boundary conditions. Here, $u$ represents the unknown velocity vector, and $p$ is the unknown pressure scalar; where $v>0$, the constant kinematic viscosity, and $g$, the body forcing term, are given.

It is well known that in both the two-dimensional and three-dimensional cases the system (1.1)-(1.4) has a steady state solution ([17], [37], [72], [73]). Moreover, for small Reynolds numbers (for example, large viscosity or small forcing) the system has a unique steady state solution which is globally stable ([54], [72]). As the Reynolds number increases, one expects that this steady state loses its stability and goes through a bifurcation (however, this scenario is not always true, see, e.g., [21], [62]). Moreover, this system could go through successive bifurcation, and exhibit chaotic and turbulent behavior (see, e.g., [59], [73]). Such turbulent dynamics is not restricted to transient solutions, but it can sustain itself for infinitely long intervals of time. To study rigorously this long-time turbulent dynamics one has to make sure that the solutions of (1.1)-(1.4) remain regular globally in time. This is known to be true in the two-dimensional case. However, in the case of three-dimensional flows this is still one of the most challenging open scientific problems. As a result, the study of the long-time behavior of the solutions and the global attractor for the system (1.1)-(1.4) has been restricted to the two-dimensional case. Indeed, the first such rigorous study was presented by Foias and Prodi in [29], where the authors have shown that the global dynamics 
is "determined" by finitely many degrees of freedom. Since then lots of work concerning this issue has been done, and we refer the reader to, for example, [16], [19], [21], [32], [33], [51] and the references therein, for the best available results in this regard.

In this paper we are interested in developing a numerical criterion for the stabilization of steady state solutions of system (1.1)-(1.4), using linear feedback control operators. The results and techniques presented here are inspired by [20] and [75].

Our main result states that if we can design a linear feedback controller that stabilizes a steady state solution to the closed-loop $m$-state Galerkin approximation or $m$-state nonlinear Galerkin approximation, where $m$ is bigger than some $M_{\mathrm{GLK}}$ (in the Galerkin case) or some $M_{\mathrm{NLG}}$ (in the nonlinear Galerkin case), then there is a nearby steady state solution to the closed-loop Navier-Stokes system with the same linear feedback controller. Our objective echoes the approach in [55]: we are concerned with "an entirely new stable system" (the closed loop system). We provide explicit bounds on $M_{\mathrm{GLK}}$ and $M_{\mathrm{NLG}}$ in terms of the physical parameters of the equations. Moreover, we show that in this case the steady state of the nonlinear Galerkin converges to the exact solutions much faster than the one of the standard Galerkin.

It is worth mentioning that all the conditions of this criterion are checkable by the computed numerical solutions, and no other conditions are imposed on the unknown exact solution. Furthermore, let us stress the fact that in general there are no error estimates for nonlinear elliptic steady state problem. This is due to the fact that such equations might have multiple solutions and even a continuum of solutions ([30], [35]). This is obvious, for instance, in the case of non-constant steady state solutions of equations with translational symmetry. However, we do not encounter this problem here because the feedback control operator isolates the steady state under consideration and makes the use of standard versions of the implicit function theorem at hand. Here we will present only the two-dimensional NSE case. Similar results are also valid in the three dimensional case, but with different dependence on the physical parameters.

This paper is organized as follows: In Section 2 on the facing page we review preliminaries and functional spaces. In Section 3 on page 44 we give a criterion for the Galerkin approximation case. In Section 4 on page 60 we provide a similar criterion for the nonlinear Galerkin approximation. In Section 5 on page 83 we show that we can use postprocessing Galerkin and nonlinear Galerkin methods to improve the accuracy. In Section 6 on page 88 we provide an algorithm for applying our numerical criterion.

This paper is dedicated to Professor Ciprian Foias on the occasion of his retirement from Indiana University, and to Professor Roger Temam on the occasion of his $60^{\text {th }}$ birthday. We would like to thank them both for their continuous support and encouragement through all over our careers. Their work is a constant source of inspiration for us. 


\section{Preliminaries AND FUnCTIONAL SETTING}

As we mentioned earlier, we consider here only the two dimensional Navier-Stokes case. Therefore we suppose that $\Omega$ is a bounded subset of $\mathbb{R}^{2}$ with smooth boundary. Let $H^{q}(\Omega)$ denote the usual Sobolev spaces of index $q$ (cf. [2]). We will denote by $|\cdot|$ the $L^{2}-$ norm, and $(\cdot, \cdot)$ the $L^{2}-$ inner product. We set

$$
\mathcal{V}=\left\{\varphi \in\left(C_{0}^{\infty}(\Omega)\right)^{2}: \nabla \cdot \varphi=0\right\} .
$$

The spaces $H, V$ will denote the closures of $\mathcal{V}$ in $L^{2}$ and $H^{1}$ respectively. We introduce the inner product

$$
((u, v))=\sum_{i=1}^{2} \int_{\Omega} \frac{\partial u}{\partial x_{i}} \frac{\partial v}{\partial x_{i}} d x, \quad \text { for } u, v \in V
$$

and denote by $\|\cdot\|$ the norm induced by the inner product $((\cdot, \cdot))$. Thanks to the Poincaré inequality, the norm $\|\cdot\|$ is equivalent to the $H^{1}$-norm on $V$. Let $P: L^{2} \rightarrow H$ be the orthogonal projection, and let $A=-P \Delta$ be the Stokes operator, subject to the homogeneous Dirichlet, no-slip, boundary condition. It is well known that $A^{-1}$ is a self-adjoint positive compact operator from $H$ onto $\mathcal{D}(A)=H^{2} \cap V$ (cf. [17], [37], [58], [73]). Let $0<\lambda_{1} \leq \lambda_{2} \leq \cdots$ be the eigenvalues of $A$, repeated according to their multiplicities, and let $\left\{\omega_{k}\right\}_{k=1}^{\infty}$ be the corresponding eigenfunctions. We denote by $H_{m}$ the linear space spanned by $\left\{\omega_{1}, \ldots, \omega_{m}\right\}$, by $P_{m}: H \rightarrow H_{m}$ the orthogonal projection, and by $Q_{m}=I-P_{m}$. Moreover, we observe that $V^{\prime}=\mathcal{D}\left(A^{-1 / 2}\right)$ is the dual space of $V$.

It is well known that the Navier-Stokes equations (NSE) (1.1)-(1.4) are equivalent to the functional differential equation (see, e.g., [17], [72])

$$
\begin{aligned}
& \frac{d u}{d t}+v A u+B(u, u)=g, \\
& u(0)=u_{\mathrm{in}},
\end{aligned}
$$

where $B(u, u)=P((u \cdot \nabla) u)$, the nonlinear (bilinear) term.

The following are well-known facts (cf. [17], [37], [58], [73])

$$
\begin{aligned}
\|v\| \geq \lambda_{1}^{1 / 2}|v|, \quad \forall v \in V^{\prime}, \\
|A v| \geq \lambda_{1}^{1 / 2}\|v\|, \quad \forall v \in \mathcal{D}(A) .
\end{aligned}
$$

There is a constant $C_{0}>0$ such that (cf. [64])

$$
\frac{m}{C_{0}} \leq \frac{\lambda_{m}}{\lambda_{1}} \leq C_{0} m
$$




$$
\begin{gathered}
(B(u, v), w)=-(B(u, v), w) ;(B(u, v), v)=0, \quad \forall u, v, w \in V, \\
|(B(u, v), w)| \leq C_{1}|u|^{1 / 2}\|u\|^{1 / 2}\|v\||w|^{1 / 2}\|w\|^{1 / 2}, \quad \forall u, v, w \in V, \\
|(B(u, v), w)| \leq C_{1}|u|^{1 / 2}|A u|^{1 / 2}\|v\||w|, \\
\forall u \in \mathcal{D}(A), \forall v \in V, \forall w \in H, \\
|(B(u, v), w)| \leq C_{1}|u|\|v\||w|^{1 / 2}|A w|^{1 / 2}, \\
\forall u \in H, \forall v \in V, \forall w \in \mathcal{D}(A), \\
|(B(u, v), w)| \leq C_{1}|u|^{1 / 2}\|u\|^{1 / 2}\|v\|^{1 / 2}|A v|^{1 / 2}|w|, \\
\forall u \in V, \forall v \in \mathcal{D}(A), \forall w \in H, \\
|(B(u, v), w)| \leq C_{1}\left(\|u\|^{1 / 2}|A u|^{1 / 2}\|v\|^{1 / 2}|A v|^{1 / 2}\right. \\
\left.\times+|u|^{1 / 2}|A u|^{1 / 2}|A v|\right)\left|A^{-1 / 2} w\right|, \\
\forall u, v \in \mathcal{D}(A), \quad \forall w \in \mathcal{D}\left(A^{-1 / 2}\right) .
\end{gathered}
$$

Next we recall the following two-dimensional version of the Lieb-Thirring inequality (cf. [60], see also [74]), for the two dimensional case.

Proposition 2.1. (Lieb-Thirring inequality) Let $\left\{\varphi_{j}\right\}_{j=1}^{N} \subset V$ be a subset of orthogonal functions in $H$ with $\left\|\varphi_{j}(x)\right\|_{L^{2}}=v$, for $j=1,2, \ldots, N$. Then there is an absolute constant $C_{2}$, which is independent of $N$, such that

$$
\int_{\Omega}\left[\sum_{j=1}^{N}\left|\varphi_{j}(x)\right|^{2}\right]^{2} d x \leq C_{2} v^{2} \sum_{j=1}^{N}\left\|\varphi_{j}(x)\right\|^{2},
$$

where $\left|\varphi_{j}(x)\right|$ denotes the absolute value of $\varphi_{j}(x)$.

The Galerkin approximation to the Navier-Stokes equations (NSE) (2.1) and (2.2), which is based on the first $m$-eigenmodes of the Stokes operator $A$, is given by the system of ordinary differential equations

$$
\begin{aligned}
& \frac{d u_{m}}{d t}+v A u_{m}+P_{m} B\left(u_{m}, u_{m}\right)=P_{m} g, \\
& u_{m}(0)=P_{m} u_{\mathrm{in}},
\end{aligned}
$$

where $u_{m}(t) \in H_{m}=\operatorname{Span}\left\{\omega_{1}, \ldots, \omega_{m}\right\}$.

Suppose that the equations (2.13) and (2.14) have a stationary solution $u_{m}^{*}$, which is unstable. Let $L_{m}: H_{m} \rightarrow H_{m}$ be a linear feedback stabilizing operator. That is, $u_{m}^{*}$ is a stable steady state solution to the closed-loop

$$
\frac{d u_{m}}{d t}+v A u_{m}+P_{m} B\left(u_{m}, u_{m}\right)+L_{m}\left(u_{m}-u_{m}^{*}\right)=P_{m} g .
$$


The above equation can be viewed as the $m$-state Galerkin approximation to the following closed-loop Navier-Stokes system

$$
\frac{d u}{d t}+v A u+B(u, u)+L_{m}\left(u-u_{m}^{*}\right)=g .
$$

As a result, the $n$-state Galerkin approximation associated with (2.16), for $n \geq$ $m$, is given by

$$
\frac{d u_{n}}{d t}+v A u_{n}+P_{n} B\left(u_{n}, u_{n}\right)+P_{n} L_{m}\left(u_{n}-u_{m}^{*}\right)=P_{n} g .
$$

The question is: provided that $m$ is large enough, is $L_{m}$ stabilizing a steady state solution $u_{n}^{*}$ of (2.17) which is nearby $u_{m}^{*}$ ? In this chapter we will give an affirmative answer to this question, provided $L_{m}$ belongs to a certain class of linear operators. Moreover, we will provide an explicit estimate on how large $m$ should be in order for the answer to be affirmative.

Motivated by the above discussion we will consider, by analogy with (2.16), the more general closed-loop NSE

$$
\begin{aligned}
& \frac{d u}{d t}+v A u+B(u, u)+\gamma L u=f, \\
& u(0)=u_{\text {in }},
\end{aligned}
$$

where $\gamma \geq 0$, is a tracking parameter, and $L$ is a linear operator which satisfies the following properties:

(i) There is a fixed $\alpha \in[0,1)$ such that $L: \mathcal{D}\left(A^{\alpha}\right) \rightarrow H$.

(ii) There is a dimensionless constant $C_{L}>0$ such that for any $\beta \in[0,1]$ we have

$$
\left|A^{\beta-\alpha} L u\right| \leq v \lambda_{1}^{1-\alpha} C_{L}^{1-\alpha}\left|A^{\beta} u\right|, \quad \forall u \in \mathcal{D}\left(A^{\beta}\right) .
$$

In particular, when $\beta=\max \left\{0, \alpha-\frac{1}{2}\right\}$ in (2.20) we have

$$
|(L u, u)| \leq v \lambda_{1}^{1-\alpha} C_{L}^{1-\alpha}|u|^{2-2 \alpha}\|u\|^{2 \alpha}, \quad \forall u \in V .
$$

Notice that for $\gamma=0$, the equation (2.18) is the NSE (1.1), and that $f$ contains the term $L_{m} u_{m}^{*}$.

The feedback linear operator $L$ is designed to stabilize the steady state of the Galerkin approximation. Therefore, roughly speaking, it stabilizes the lower modes. However, when it is introduced into the full PDE it could destabilize (excite) the higher modes (a phenomenon known as spillover). The above technical conditions on the operator $L$ simply mean that the viscous linear term $v A$ in the closed-loop system is stronger than $\gamma L$, and hence will "suppress" this potential spillover phenomenon. 


\section{A STABILIZATION CRITERION FOR THE STANDARD GALERKIN APPROXIMATION CASE}

The Galerkin approximation equation of the closed-loop NSE based on $m$ eigenmodes of the Stokes operator is (cf. [17], [37], [58], [73])

$$
\begin{aligned}
& \frac{d u_{m}}{d t}+v A u_{m}+P_{m} B\left(u_{m}, u_{m}\right)+\gamma P_{m} L u_{m}=P_{m} f, \\
& u_{m}(0)=P_{m} u_{\mathrm{in}},
\end{aligned}
$$

where $u_{m} \in H_{m}$.

Following the notation in [20] and [75] we denote, for $1 \leq m \leq \infty$, by $u_{m}^{\infty}$ the stationary solution of the equations (3.1), and by $u^{\infty} \equiv u_{\infty}^{\infty}$ the steady state of the equations (2.18). Furthermore, we also denote by $\mathcal{A}_{m}\left(u_{0}\right): H_{m} \rightarrow H_{m}$ the linearized operator about $u_{0} \in \mathcal{D}(A)$, defined as

$$
\mathcal{A}_{m}\left(u_{0}\right) v=v A v+P_{m}\left(B\left(u_{0}, v\right)+B\left(v, u_{0}\right)\right)+\gamma P_{m} L v, \quad \forall v \in H_{m}
$$

When $m=\infty$, we will denote by $\mathcal{A}=\mathcal{A}_{\infty}$. We will denote $\operatorname{Sp}\left(\mathcal{A}_{m}\left(u_{0}\right)\right)$ the spectrum of $\mathcal{A}_{m}\left(u_{0}\right) ; B(E, \rho)=\{z \in \mathbb{C}: \operatorname{dist}(z, E)<\rho\}$, for any subset $E \subset \mathbb{C}$; and by $(E)_{\sigma}=\{z \in E:|\mathfrak{R} z| \leq \sigma\}$. Notice that our definition of $(E)_{\sigma}$ is different than the one in [20] and [75].

In the following Lemma we follow closely the papers [20] and [75]. However, we adjust the details to take into consideration the effect of the feedback operator $L$.

Lemma 3.1. Let $1 \leq m \leq \infty$, let $u_{m}^{\infty} \in V$ be a steady state solution of the equation (3.1).

(i) If

$$
\left|u_{m}^{\infty}\right| \leq K_{1}
$$

then

$$
\begin{aligned}
&\left\|u_{m}^{\infty}\right\| \leq K_{2}=\left(\frac{v \lambda_{1} G K_{1}}{1-\alpha}\right)^{1 / 2}+\gamma^{1 /[2(1-\alpha)]} C_{L}^{1 / 2} \lambda_{1}^{1 / 2} K_{1}, \\
&\left|A u_{m}^{\infty}\right| \leq \lambda_{1}^{1 / 2} K_{3}=\frac{8 v \lambda_{1} G}{1-\alpha}+\frac{32^{1 / 2} C_{1}^{2} K_{1} K_{2}^{2}}{v^{2}(1-\alpha)^{2}}+8^{1 / 2} \gamma^{1 /(1-\alpha)} C_{L} \lambda_{1} K_{1},
\end{aligned}
$$

where $G=|f| /\left(\lambda_{1} v^{2}\right)$, the Grashoff number.

(ii) If $\gamma C_{L}^{1 / 2}<1$, then

$$
\begin{aligned}
& \left\|u_{m}^{\infty}\right\| \leq \bar{K}_{2}=\frac{|f|}{v \lambda_{1}^{1 / 2}\left(1-\gamma C_{L}^{1 / 2}\right)} \\
& \left|A u_{m}^{\infty}\right| \leq \lambda_{1}^{1 / 2} \bar{K}_{3}=\frac{8 v \lambda_{1} G}{1-\alpha}+\frac{32^{1 / 2} C_{1}^{2} \bar{K}_{2}^{3}}{v^{2} \lambda_{1}^{1 / 2}(1-\alpha)^{2}}+8^{1 / 2} \gamma^{1 /(1-\alpha)} C_{L} \lambda_{1}^{1 / 2} \bar{K}_{2}
\end{aligned}
$$


Notice in particular that $\gamma=0$, i.e., the open-loop case falls into this situation.

Proof. Let $u_{m}^{\infty}$ be a stationary solution of the equation (3.1) with $\left|u_{m}^{\infty}\right| \leq K_{1}$. Taking the inner product of the equation (3.1) with $u_{m}^{\infty}$ in $H_{m}$, and using (2.6), we get

$$
v\left\|u_{m}^{\infty}\right\|^{2} \leq|f|\left|u_{m}^{\infty}\right|+\gamma \nu C_{L}^{1-\alpha} \lambda_{1}^{1-\alpha}\left|u_{m}^{\infty}\right|^{2-2 \alpha}\left\|u_{m}^{\infty}\right\|^{2 \alpha} .
$$

By Young's inequality, we obtain

$$
\left\|u_{m}^{\infty}\right\|^{2} \leq \frac{|f| K_{1}}{v(1-\alpha)}+\gamma^{1 /(1-\alpha)} C_{L} \lambda_{1} K_{1}^{2} .
$$

Thus we have proved the estimate (3.5). Taking the inner product of equation (3.1) with $A u_{m}^{\infty}$ in $H_{m}$ gives us

$$
v\left|A u_{m}^{\infty}\right|^{2} \leq|f|\left|A u_{m}^{\infty}\right|+\left|\left(B\left(u_{m}^{\infty}, u_{m}^{\infty}\right), A u_{m}^{\infty}\right)\right|+\gamma\left|\left(L u_{m}^{\infty}, A u_{m}^{\infty}\right)\right| .
$$

By using the Cauchy-Schwarz inequality, and (2.8) and (2.20) with $\beta=\alpha$, we reach

$$
\begin{aligned}
v\left|A u_{m}^{\infty}\right|^{2} \leq|f|\left|A u_{m}^{\infty}\right|+C_{1}\left|u_{m}^{\infty}\right|^{1 / 2}\left\|u_{m}^{\infty}\right\|\left|A u_{m}^{\infty}\right|^{3 / 2} & \\
& +\gamma \nu C_{L}^{1-\alpha} \lambda_{1}^{1-\alpha}\left|u_{m}^{\infty}\right|^{1-\alpha}\left|A u_{m}^{\infty}\right|^{1+\alpha} .
\end{aligned}
$$

Thanks to Young's inequality, we get

$$
\left|A u_{m}^{\infty}\right| \leq \frac{8|f|}{v(1-\alpha)}+\frac{32^{1 / 2} C_{1}^{2} K_{1} K_{2}^{2}}{v^{2}(1-\alpha)^{2}}+8^{1 / 2} \gamma^{1 /(1-\alpha)} C_{L} \lambda_{1} K_{1},
$$

which completes the proof part (i) of the lemma. The proof of the part (ii) of the lemma is similar to the one in the case of $\gamma=0$, i.e., the NSE. We refer to [17], [73] for details.

Remark. The condition $\gamma C_{L}^{1 / 2}<1$ in part (ii) of the above lemma means that the linear instabilities (about zero) that might be caused by the linear feedback operator, $L$, are weak and can be suppressed by the viscous term $A$.

Lemma 3.2. Let $R>0, u_{0} \in \mathcal{D}(A)$ with $\left|A u_{0}\right| \leq \lambda_{1}^{1 / 2} R$. If $w$ is an eigenfunction of $\mathcal{A}_{m}\left(u_{0}\right)$, then $w \in \mathcal{D}(A)$. Moreover, if $|w|=v$ and $\mathcal{A}_{m}\left(u_{0}\right) w=\Lambda w$ for some $\Lambda \in \mathbb{C}$, then we have

$$
\begin{aligned}
\|w\| & \leq K_{4}(\gamma, \Lambda, v, R), \\
|A w| & \leq K_{5}(\gamma, \Lambda, v, R),
\end{aligned}
$$


where

$$
K_{4}(\gamma, \Lambda, v, R)=\left(\frac{2 \nu|\Lambda|}{1-\alpha}+2 \gamma^{1 /(1-\alpha)} v^{2} \lambda_{1} C_{L}\right)^{1 / 2}+\frac{C_{1} R}{1-\alpha}
$$

and

$$
\begin{aligned}
K_{5}(\gamma, \Lambda, \nu, R)=\frac{|\Lambda|}{1-\alpha} & +\frac{C_{1} R K_{4}(\gamma, \Lambda, \nu, R)}{\nu(1-\alpha)} \\
& +\frac{C_{1} \lambda_{1}^{1 / 4} R K_{4}^{1 / 2}(\gamma, \Lambda, \nu, R)}{1-\alpha}+\gamma^{1 /(1-\alpha)} \nu C_{L} \lambda_{1} .
\end{aligned}
$$

Proof. Let $|w|=v$ and

$$
\mathcal{A}_{m}\left(u_{0}\right) w=\Lambda w
$$

Taking the inner product of the above equation with $w$ in $H_{m}$, and using (2.6) leads to

$$
v\|w\|^{2} \leq|\Lambda||w|^{2}+\left|\left(B\left(w, u_{0}\right), w\right)\right|+\gamma|(L w, w)| .
$$

The inequalities (2.7) and (2.21) give us

$$
v\|w\|^{2} \leq|\Lambda||w|^{2}+C_{1}\left\|u_{0}\right\||w|\|w\|+\gamma \nu C_{L}^{1-\alpha} \lambda_{1}^{1-\alpha}|w|^{2-2 \alpha}\|w\|^{2 \alpha} .
$$

By Young's inequality and the Cauchy-Schwarz inequality, we get

$$
\|w\|^{2} \leq \frac{2 \nu|\Lambda|}{1-\alpha}+\frac{C_{1}^{2} R^{2}}{(1-\alpha)^{2}}+2 \gamma^{1 /(1-\alpha)} v^{2} \lambda_{1} C_{L},
$$

which leads to (3.9). Now by taking the $L^{2}$-norm in the equation (3.13), we reach

$$
v|A w| \leq|\Lambda||w|+\left|B\left(u_{0}, w\right)\right|+\left|B\left(w, u_{0}\right)\right|+\gamma|L w|
$$

By using (2.8), (2.10), and (2.20) with $\beta=\alpha$, we get

$$
\begin{array}{r}
v|A w| \leq|\Lambda||w|+C_{1}\left|u_{0}\right|^{1 / 2}\left|A u_{0}\right|^{1 / 2}\|w\|+C_{1}|w|^{1 / 2}\|w\|^{1 / 2}\left\|u_{0}\right\|^{1 / 2}\left|A u_{0}\right|^{1 / 2} \\
+\gamma v C_{L}^{1-\alpha} \lambda_{1}^{1-\alpha}|w|^{1-\alpha}|A w|^{\alpha}
\end{array}
$$

Following Young's inequality, we conclude that

$$
\begin{aligned}
|A w| \leq \frac{|\Lambda|}{1-\alpha}+\frac{C_{1} R K_{4}(\gamma, \Lambda, \nu, R)}{\nu(1-\alpha)}+\frac{C_{1} \lambda_{1}^{1 / 4} R K_{4}^{1 / 2}(\gamma, \Lambda, \nu, R)}{1-\alpha} & +\gamma^{1 /(1-\alpha)} v C_{L} \lambda_{1}
\end{aligned}
$$


Lemma 3.3. Let $R>0, u_{0} \in V$ with $\left\|u_{0}\right\| \leq R$ and $s \in \mathbb{R}$, and let $M_{1}(s)$ be the number of the eigenvalues of $\mathcal{A}_{m}\left(u_{0}\right)$ whose real parts are less than or equal to $s$, i.e.,

$$
M_{1}(s)=\# \text { of }\left\{\lambda \in \operatorname{Sp}\left(\mathcal{A}_{m}\left(u_{0}\right)\right): \mathfrak{R} \lambda<s\right\}
$$

Then we have

$$
M_{1}(s) \leq K_{6}(\gamma, s, v, R),
$$

where

$$
\begin{aligned}
K_{6}(\gamma, s, v, R)=\frac{1}{2}\left\{\left(\frac{4 s C_{0}}{v \lambda_{1}(1-\alpha)}-1\right)+\left[\left(\frac{4 s C_{0}}{v \lambda_{1}(1-\alpha)}-1\right)^{2}\right.\right. & \\
& \left.\left.+\frac{8 C_{0} C_{2} R^{2}}{v^{2} \lambda_{1}(1-\alpha)^{2}}+16 \gamma^{1 /(1-\alpha)} C_{0} C_{L}\right]^{1 / 2}\right\} .
\end{aligned}
$$

Proof. Let $m<\infty$ be finite, and let $\Lambda_{1}, \ldots, \Lambda_{K}$ be the eigenvalues of $\mathcal{A}_{m}\left(u_{0}\right)$, repeated according to their multiplicities, such that $\mathfrak{R} \Lambda_{j} \leq s$, for $j=1,2, \ldots, K$. We will denote by $E_{K}$ the linear space spanned by the generalized eigenfunctions of $\mathcal{A}_{m}\left(u_{0}\right)$ corresponding to the eigenvalues $\Lambda_{1}, \ldots, \Lambda_{K}$. Let $\psi_{1}, \ldots, \psi_{K}$ be an orthogonal basis of $E_{K}$ with $\left|\psi_{j}\right|=v$ for $j=1,2, \ldots, K$, and let $\Pi_{K}: H_{m} \rightarrow E_{K}$ be the orthogonal projection. Then we get

$$
\mathfrak{R}\left(\sum_{j=1} K \Lambda_{j}\right)=\mathfrak{R}\left\{\operatorname{trace}\left(\Pi_{K} \mathcal{A}_{m}\left(u_{0}\right) \Pi_{K}\right)\right\}=v^{-2} \mathfrak{R}\left(\sum_{j=1} K\left(\mathcal{A}_{m}\left(u_{0}\right) \psi_{j}, \psi_{j}\right)\right) .
$$

By using (2.6) and (2.21), we reach

$$
\begin{aligned}
& \mathfrak{R}\left(\sum_{j=1} K \Lambda_{j}\right) \\
& \quad=v^{-2} \mathfrak{R}\left\{\sum_{j=1} K\left[v\left(A \psi_{j}, \psi_{j}\right)+\left(B\left(\psi_{j}, u_{0}\right), \psi_{j}\right)+\gamma\left(L \psi_{j}, \psi_{j}\right)\right]\right\} \\
& \quad \geq v^{-2} \sum_{j=1} K\left[v\left\|\psi_{j}\right\|^{2}-\left|\left(B\left(\psi_{j}, u_{0}\right), \psi_{j}\right)\right|-\gamma v C_{L}^{1-\alpha} \lambda_{1}^{1-\alpha}\left|\psi_{j}\right|^{2-2 \alpha}\left\|\psi_{j}\right\|^{2 \alpha}\right] .
\end{aligned}
$$

By using the Cauchy-Schwarz and Lieb-Thirring (2.12) inequalities, we obtain

$$
\begin{aligned}
\left|\sum_{j=1}^{K}\left(B\left(\psi_{j}, u_{0}\right), \psi_{j}\right)\right| & \leq\left(\int_{\Omega}\left(\sum_{j=1} K \psi_{j}^{2}(x)\right)^{2} d x\right)^{1 / 2}\left\|u_{0}\right\| \\
& \leq\left(C_{2} v^{2} \sum_{j=1} K\left\|\psi_{j}\right\|^{2}\right)^{1 / 2}\left\|u_{0}\right\| \\
& \leq \frac{v(1-\alpha)}{2}\left(\sum_{j=1} K\left\|\psi_{j}\right\|^{2}\right)+\frac{C_{2} v\left\|u_{0}\right\|^{2}}{2(1-\alpha)} .
\end{aligned}
$$


On the other hand, and by Young's inequality, we have

$$
\gamma \nu C_{L}^{1-\alpha} \lambda_{1}^{1-\alpha}\left|\psi_{j}\right|^{2-2 \alpha}\left\|\psi_{j}\right\|^{2 \alpha} \leq \nu \alpha\left\|\psi_{j}\right\|^{2}+\nu(1-\alpha) \gamma^{1 /(1-\alpha)} C_{L} \lambda_{1}\left|\psi_{j}\right|^{2},
$$

that is

$$
\gamma \nu C_{L}^{1-\alpha} \lambda_{1}^{1-\alpha}\left|\psi_{j}\right|^{2-2 \alpha}\left\|\psi_{j}\right\|^{2 \alpha} \leq \nu \alpha\left\|\psi_{j}\right\|^{2}+\nu^{3}(1-\alpha) \gamma^{1 /(1-\alpha)} C_{L} \lambda_{1} .
$$

Moreover,

$$
\sum_{j=1} K\left\|\psi_{j}\right\|^{2}=\sum_{j=1} K\left(A \psi_{j}, \psi_{j}\right) \geq v^{2} \sum_{j=1} K \lambda_{j}
$$

Therefore,

$$
\begin{aligned}
K s \geq \mathfrak{R}\left(\sum_{j=1} K \Lambda_{j}\right) \geq & \frac{v(1-\alpha)}{2} \sum_{j=1} K \lambda_{j}-\frac{C_{2}\left\|u_{0}\right\|^{2}}{2 v(1-\alpha)} \\
& -\sum_{j=1} K \nu(1-\alpha) \gamma^{1 /(1-\alpha)} C_{L} \lambda_{1} .
\end{aligned}
$$

By using(2.5), we get

$$
K s \geq \frac{v \lambda_{1}(1-\alpha)}{2 C_{0}} \frac{K(K+1)}{2}-\frac{C_{2}\left\|u_{0}\right\|^{2}}{2 v(1-\alpha)}-v(1-\alpha) \gamma^{1 /(1-\alpha)} C_{L} \lambda_{1} K,
$$

which implies (3.14).

Remark. In a sense, the estimate (3.14) is sharp because the constant $K_{6}(\gamma, s, v, R)$ is growing linearly in $s$. For example, in the case when $u_{0}=0$ and $\gamma=0$ we have $\mathcal{A}\left(u_{0}\right)=v A$, and in this case the $\Lambda_{j}=\lambda_{j}$ behaves like $j$ asymptotically (see (2.5)). Thus, in this special case, $M_{1}(s) \sim s$.

3.1. Estimates for the resolvents in the Galerkin case. For every $R>0$ we define with Prodi [67] the parabola-like set $\sigma(R) \subset \mathbb{C}$ by setting

$$
\sigma(R)=\left\{z \in \mathbb{C}: \exists x \geq \lambda_{1},|z-v x| \leq C_{1} R x^{1 / 2}+\gamma \nu \lambda_{1}^{1-\alpha} C_{L}^{1-\alpha} x^{\alpha}\right\},
$$

where $C_{1}$ and $C_{L}$ are as in (2.7) and (2.21) respectively. It is worth mentioning that when one deals with three dimensional Navier-Stokes equations, the set $\sigma(R)$ is bigger, but it has the same properties as far as the spectra of the operator $\mathcal{A}_{m}\left(u_{0}\right)$, for $\left\|u_{0}\right\| \leq R$ and $1 \leq m \leq \infty$, is concerned.

As in [20] and [75], one can show that the spectrum of $\mathcal{A}_{m}\left(u_{0}\right)$ consists of isolated eigenvalues of finite multiplicities. The following lemmas are similar to those of [20] and [75], but we add the contribution of the linear control operator $L$. The proofs will be omitted here, and can be found for the case where $L=0$ in [20]. 
Lemma 3.4. Let $R>0, u_{0} \in V$ with $\left\|u_{0}\right\| \leq R$. Then

- the spectra of $\mathcal{A}_{m}\left(u_{0}\right), m=1, \ldots, \infty$, are in $\sigma(R)$,

- for every $z \notin \sigma(R)$ the following inequalities hold:

(3.17) $\left|\left(\mathcal{A}_{m}\left(u_{0}\right)-z\right)^{-1} v\right| \leq \operatorname{dist}(z, \sigma(R))^{-1}|v|=K_{H}(\gamma, v, R, z)|v|$,

(3.18) $\left\|\left(\mathcal{A}_{m}\left(u_{0}\right)-z\right)^{-1} v\right\| \leq K_{V}(\gamma, v, R, z)|v|, \quad \forall v \in H_{m}$,

(3.19) $\left|A\left(\mathcal{A}_{m}\left(u_{0}\right)-z\right)^{-1} v\right| \leq K_{A}(\gamma, v, R, z)|v|, \quad \forall v \in H_{m}$,

where

$$
\begin{aligned}
K_{V}(\gamma, v, R, z)= & {\left[\left(2 \lambda_{1} \gamma^{1 /(1-\alpha)}+\frac{C_{1}^{2} R^{2}}{(1-\alpha)^{2} v^{2}}\right.\right.} \\
& \left.\left.+\frac{2|z|}{(1-\alpha) v}\right) K_{H}^{2}(\gamma, v, R, z)+\frac{2 K_{H}(\gamma, v, R, z)}{(1-\alpha) v}\right]^{1 / 2}, \\
K_{A}(\gamma, v, R, z)= & K_{H}(\gamma, v, R, z)\left[\frac{4}{v(1-\alpha)}+\left(\frac{8|z|}{(1-\alpha) v}\right.\right. \\
& \left.\left.+\frac{16 C_{1}^{2} R^{2}}{v^{2}(1-\alpha)^{2}}+4 \gamma^{2 /(1-\alpha)} C_{L}^{2} \lambda_{1}^{2}\right)^{1 / 2} K_{V}(\gamma, v, R, z)\right] .
\end{aligned}
$$

Following [75], we will use Carleman's inequality for the Hilbert-Schmidt (HS) operators to prove this section's main result, Lemma 3.6 on the next page. First let us recall some well known facts about H-S operators (cf. [24])

Theorem 3.1. Let $\mathcal{H}$ be a Hilbert space, $F: \mathcal{H} \rightarrow \mathcal{H}$ be an $H-S$ operator with non-zero eigenvalues $\mu_{1}, \mu_{2}, \ldots$, repeated according to their multiplicities. Then

(i) the following infinite product

$$
\varphi_{\lambda}(F)=\prod_{k \geq 1}\left(1-\frac{\mu_{k}}{\lambda}\right) e^{\mu_{k} / \lambda}
$$

converges and defines an analytic function for every $\lambda \neq 0, \lambda \notin \operatorname{Sp}(F)$;

(ii) (Carleman's inequality) the following inequality

$$
\left.\| \varphi_{\lambda}(F) \lambda I-F\right)^{-1} \|_{\mathcal{L}(\mathcal{H}, \mathcal{H})} \leq|\lambda| e^{1 / 2\left[1+\left(\|F\|_{2} /|\lambda|\right)^{2}\right]}
$$

holds for every $\lambda \neq 0, \lambda \notin \mathrm{Sp}(F)$, where $\|F\|_{2}$ denotes the $H$-S norm;

(iii) for every $T \in \mathcal{L}(\mathcal{H}, \mathcal{H})$, TF and $F T$ are $H$-S operators. Moreover,

$$
\max \left\{\|F T\|_{2},\|T F\|_{2}\right\} \leq\|F\|_{2}\|T\|_{\mathcal{L}(\mathcal{H}, \mathcal{H})} .
$$


50 Chongsheng CaO, IoAnnis G. Kevrekidis \& Edriss S. Titi

We recall the following Lemma, which was presented in [75]. We refer to [75] for details of the proof.

Lemma 3.5. Let $R>0, u_{0} \in V$ with $\left\|u_{0}\right\| \leq R$. Then for every $z \notin \sigma(R)$ the operators $\left(\mathcal{A}_{m}\left(u_{0}\right)-z\right)^{-1}$, for $m=1, \ldots, \infty$, are H-S operators. Moreover,

$$
\left\|\left(\mathcal{A}_{m}\left(u_{0}\right)-z\right)^{-1}\right\|_{2} \leq K_{H S}(\gamma, v, R, z)
$$

where

$$
K_{H S}(\gamma, \nu, R, z)=\frac{C_{0} \pi}{\sqrt{6} \lambda_{1}} K_{A}(\gamma, \nu, R, z)
$$

where $K_{A}(\gamma, v, R, z)$ is as in (3.21).

Lemma 3.6. Let $R>0, u_{0} \in \mathcal{D}(A)$, with $\left|A u_{0}\right| \leq \lambda_{1}^{1 / 2} R$ and $\rho, s \geq 0$, and let $\lambda \in \mathbb{C}$ and satisfy $|\lambda| \leq s$. Let

$$
\lambda_{c}=-\frac{C_{1}^{2} R^{2}}{4 v(1-\alpha)}-\gamma^{1 /(1-\alpha)} v(1-\alpha) C_{L} \lambda_{1} .
$$

Then for every $m<\infty$, if

$$
\operatorname{dist}\left(\lambda, \operatorname{Sp}\left(\mathcal{A}_{m}\left(u_{0}\right)\right)\right) \geq \rho,
$$

then we have

$$
\begin{aligned}
\left|\left(\mathcal{A}_{m}\left(u_{0}\right)-\lambda\right)^{-1} v\right| \leq K_{7}(\gamma, \rho, s, v, R)|v|, & \forall v \in H_{m}, \\
\left\|\left(\mathcal{A}_{m}\left(u_{0}\right)-\lambda\right)^{-1} v\right\| \leq K_{8}(\gamma, \rho, s, v, R)|v|, & \forall v \in H_{m}, \\
\left|A\left(\mathcal{A}_{m}\left(u_{0}\right)-\lambda\right)^{-1} v\right| \leq K_{9}(\gamma, \rho, s, v, R)|v|, & \forall v \in H_{m},
\end{aligned}
$$

where

$$
\begin{aligned}
K_{7}(\gamma, \rho, s, \nu, R) & \lambda \notin \sigma\left(R-\lambda_{c} / 2\right), \\
\exp \left\{\frac{1}{2}+\frac{5}{2}\left|s-2 \lambda_{c}\right|^{2} K_{H S}^{2}\left(\gamma, \nu, R,\left|s-2 \lambda_{c}\right|\right)\right\} & \\
\times \frac{1}{\lambda_{c}\left|\lambda-2 \lambda_{c}\right|^{2}}\left(\frac{2\left|s-\lambda_{c}\right|}{\rho}\right)^{\widetilde{M}}, & \lambda \in \sigma\left(R-\lambda_{c} / 2\right),
\end{aligned}
$$


(3.30) $K_{8}(\gamma, \rho, s, \nu, R)$

$$
\begin{aligned}
=\left[\left(2 \lambda_{1} \gamma^{1 /(1-\alpha)}+\frac{C_{1}^{2} R^{2}}{(1-\alpha)^{2} \nu^{2}}++\frac{2 s}{(1-\alpha) v}\right) K_{7}^{2}(\gamma, \nu, R, z)\right. \\
\left.+\frac{2 K_{7}(\gamma, v, R, z)}{(1-\alpha) v}\right]^{1 / 2},
\end{aligned}
$$

(3.31) $K_{9}(\gamma, \rho, s, \nu, R)$

$$
\begin{array}{r}
=\left[\left(\frac{8 s}{(1-\alpha) v}+\frac{16 C_{1}^{2} R^{2}}{v^{2}(1-\alpha)^{2}}+4 \gamma^{2 /(1-\alpha)} C_{L}^{2} \lambda_{1}^{2}\right)^{1 / 2} K_{8}(\gamma, v, R, z)\right. \\
\left.+\frac{4}{v(1-\alpha)}\right] K_{7}(\gamma, v, R, z),
\end{array}
$$

where

$$
\widetilde{M}=K_{6}\left(\gamma, 2 \lambda_{c}+\frac{2}{\left|\lambda_{c}\right|}, \nu, R\right),
$$

$\lambda_{c}$ is defined earlier in (3.24), and $K_{H S}$ is as in (3.23).

Proof. By applying Lemma 3.4 on page 49 , we only consider the case of $\lambda \in$ $\sigma\left(R-\lambda_{c} / 2\right)$. One can check that $2 \lambda_{c} \notin \sigma(R)$. Thus, by Lemma 3.5, $F_{m}=$ $\left(\mathcal{A}_{m}\left(u_{0}\right)-2 \lambda_{c}\right)^{-1}$ is an H-S operator. On the other hand, we can write

$$
\begin{aligned}
\left(\mathcal{A}_{m}\left(u_{0}\right)-\lambda\right)^{-1}=\left(\lambda-2 \lambda_{c}\right)^{-1}\left(\mathcal{A}_{m}\right. & \left.\left(u_{0}\right)-2 \lambda_{c}\right)^{-1} \\
& \times\left(\left(\lambda-2 \lambda_{c}\right)^{-1} I-\left(\mathcal{A}_{m}\left(u_{0}\right)-2 \lambda_{c}\right)^{-1}\right)^{-1} .
\end{aligned}
$$

Notice that $\left(\lambda-2 \lambda_{c}\right)^{-1} \notin S p\left(F_{m}\right)$. We apply Lemma 3.4 on page 49 and the Carleman inequality to get

$$
\begin{aligned}
& \left\|\left(\mathcal{A}_{m}\left(u_{0}\right)-\lambda\right)^{-1}\right\|_{\mathcal{L}\left(\mathcal{H}_{m}, \mathcal{H}_{m}\right)} \\
& \quad \leq \frac{1}{\lambda_{c}\left|\lambda-2 \lambda_{c}\right|^{2}}\left|\varphi_{\lambda-2 \lambda_{c}}\left(F_{m}\right)\right|^{-1} e^{1 / 2\left[1+\left\|F_{m}\right\|_{2}^{2}\left(\left|\lambda-2 \lambda_{c}\right|^{2}\right]\right.} .
\end{aligned}
$$

Now we apply Lemma 3.5 on the facing page and the estimate for $\left|\varphi_{\lambda-2 \lambda_{c}}\left(F_{m}\right)\right|$ in [75] (see [75] for details), and we have

$$
\left|\left(\mathcal{A}_{m}\left(u_{0}\right)-\lambda\right)^{-1} v\right| \leq K_{7}(\gamma, \rho, s, v, R)|v|,
$$

where $K_{7}(\gamma, \rho, s, v, R)$ is as in (3.29).

Now we show the estimates (3.27) and (3.28). For each $g \in H_{m}$, let $\left(\mathcal{A}_{m}\left(u_{0}\right)-\right.$ $\lambda)^{-1} g=v$, i.e., 


$$
v A v+P_{m}\left(B\left(u_{0}, v\right)+B\left(v, u_{0}\right)+\gamma P_{m} L v\right)-\lambda v=g .
$$

Taking the inner product of equation (3.32) with $v$ in $H$, we obtain

$$
v\|v\|^{2} \leq|\lambda||v|^{2}+|(g, v)|+\left|\left(B\left(v, u_{0}\right), v\right)\right|+\gamma|(L v, v)| .
$$

By using (2.8) and (2.21), we reach

$$
v\|v\|^{2} \leq\left. s|v|^{2}|+| g|| v\left|+C_{1}\left\|u_{0}\right\|\right| v\left|\|v\|+\gamma \nu C_{L}^{1-\alpha} \lambda_{1}^{1-\alpha}\right| v\right|^{2-2 \alpha}\|v\|^{2 \alpha} .
$$

Thanks to Young's inequality and the estimate (3.26), we get

$$
\begin{aligned}
\frac{v(1-\alpha)}{2}\|v\|^{2} & \leq s|v|^{2}+|g||v|+\frac{C_{1}^{2}\left\|u_{0}\right\|^{2}|v|^{2}}{2 v(1-\alpha)}+\gamma^{1 /(1-\alpha)} v(1-\alpha) C_{L} \lambda_{1}|v|^{2} \\
\leq & {\left[\left(s+\frac{C_{1}^{2} R^{2}}{2 v(1-\alpha)}+\gamma^{1 /(1-\alpha)} v C_{L} \lambda_{1}\right) K_{7}^{2}(\gamma, \rho, s, \nu, R)\right.} \\
& \left.+K_{7}(\gamma, \rho, s, \nu, R)\right]|g|^{2},
\end{aligned}
$$

which implies (3.27). Moreover, taking the inner product of (3.32) with $A v$ in $H$ gives us

$$
\begin{aligned}
v|A v|^{2} \leq|\lambda|\|v\|^{2}+|(g, A v)|+\left|\left(B\left(v, u_{0}\right), A v\right)\right| & \\
& +\left|\left(B\left(u_{0}, v\right), A v\right)\right|+\gamma|(L v, A v)| .
\end{aligned}
$$

By using (2.9), (2.10), and (2.20) with $\beta=\alpha$, we get

$$
\begin{aligned}
v|A v|^{2} \leq s\|v\|^{2} & +|g||A v|+C_{1}\left\|u_{0}\right\|^{1 / 2}\left|A u_{0}\right|^{1 / 2}|v|^{1 / 2}\|v\|^{1 / 2}|A v| \\
& +C_{1}\left|u_{0}\right|^{1 / 2}\left|A u_{0}\right|^{1 / 2}\|v\||A v|+\gamma v C_{L}^{1-\alpha} \lambda_{1}^{1-\alpha}|v|^{1-\alpha}|A v|^{1+\alpha} .
\end{aligned}
$$

By Young's inequality again, and the estimates (3.26) and (3.27), we reach

$$
\begin{aligned}
\frac{v(1-\alpha)}{8}|A v|^{2} \leq s\|v\|^{2} & +\frac{2|g|^{2}}{v(1-\alpha)}+\frac{2 C_{1}^{2}\left\|u_{0}\right\|\left|A u_{0}\right||v|\|v\|}{v(1-\alpha)} \\
& +\frac{2 C_{1}^{2}\left|u_{0}\right|\left|A u_{0}\right|\|v\|^{2}}{v(1-\alpha)}+\gamma^{2 /(1-\alpha)} v \frac{(1-\alpha)}{2} C_{L}^{2} \lambda_{1}^{2}|v|^{2} \\
\leq & {\left[\frac{2}{v(1-\alpha)}+\left(s+\frac{4 C_{1}^{2} R^{2}}{2 v(1-\alpha)}+\gamma^{2 /(1-\alpha)} v \frac{(1-\alpha)}{2} C_{L}^{2} \lambda_{1}^{2}\right)\right] } \\
& \left.\times K_{8}^{2}(\gamma, \rho, s, v, R)\right]|g|^{2},
\end{aligned}
$$


which implies (3.28) and completes the proof.

We now show that the spectra of $\mathcal{A}_{m}\left(u_{0}\right)$ are, in some sense, close to each other when $m$ is large enough.

Lemma 3.7. Let $R>0, u_{0}, u_{1} \in \mathcal{D}(A)$ with $\left|A u_{0}\right| \leq \lambda_{1}^{1 / 2} R,\left|A u_{1}\right| \leq$ $\lambda_{1}^{1 / 2} R$, and let s, $\rho>0$. Then there exist a constant

$$
\begin{aligned}
\eta(\gamma, s, \rho, \nu, R)=\frac{v}{3 C_{1} K_{5}^{1 / 2}(\gamma, s, \nu, R) K_{7}(\gamma, \rho, s, \nu, R)} \\
\times \frac{\lambda_{1}^{1 / 4}}{K_{4}^{1 / 2}(\gamma, s, \nu, R)+\lambda_{1}^{1 / 4}},
\end{aligned}
$$

and a constant

(3.34) $M_{2}(\gamma, \rho, s, v, R)$

$$
\begin{aligned}
=\max & \left\{\frac{C_{L}}{C_{0}}\left(4 \gamma K_{5}(\gamma, s, \nu, R) K_{7}(\gamma, \rho, s, \nu, R)\right)^{1 /(1-\alpha)},\right. \\
& \left.\frac{2 C_{0}}{v}\left(2 C_{1} R K_{5}(\gamma, s, \nu, R) K_{7}(\gamma, \rho, s, \nu, R)+K_{4}(\gamma, s, \nu, R)\right)\right\},
\end{aligned}
$$

where $K_{4}(\gamma, s, \nu, R), K_{5}(\gamma, s, \nu, R)$, and $K_{7}(\gamma, \rho, s, \nu, R)$ are as in (3.11), (3.12), and (3.29), respectively, such that if $\left\|u_{0}-u_{1}\right\| \leq \eta(\gamma, \rho, s, \nu, R)$, and the condition

$$
\left(\operatorname{Sp}\left(\mathcal{A}_{m}\left(u_{0}\right)\right)\right)_{s}=\varnothing
$$

holds for some $m \in\left[M_{2}(\gamma, \rho, s, \nu, R), \infty\right)$, then for every $n \in[m, \infty]$ we have

$$
\left(\operatorname{Sp}\left(\mathcal{A}_{n}\left(u_{1}\right)\right)\right)_{s} \subset B\left(\operatorname{Sp}\left(\mathcal{A}_{m}\left(u_{0}\right)\right), \rho\right) .
$$

Proof. Let $n \geq m$, and let $\lambda \in\left(\operatorname{Sp}\left(\mathcal{A}_{n}\left(u_{1}\right)\right)\right)_{s}$, i.e., there is a $w \in H_{n}$, $|w|=v$ such that $\mathcal{A}_{n}\left(u_{1}\right) w=\lambda w$ and $|\mathfrak{R} \lambda| \leq s$. Denote by

$$
w_{m}=P_{m} w \quad \text { and } \quad \widetilde{w}_{m}=Q_{m} w,
$$

where $P_{m}$ and $Q_{m}$ are the orthogonal projections defined in Section 2 on page 41 . Suppose that $\lambda \notin B\left(\operatorname{Sp}\left(\mathcal{A}_{m}\left(u_{0}\right)\right), \rho\right)$, i.e., $\operatorname{dist}\left(\lambda, \operatorname{Sp}\left(\mathcal{A}_{m}\left(u_{0}\right)\right)\right) \geq \rho$. Then by Lemma 3.6 on page 50 , we get

$$
\left|\mathcal{A}_{m}\left(u_{0}\right) w_{m}-\lambda w_{m}\right| \geq \frac{\left|w_{m}\right|}{K_{7}(\gamma, \rho, s, \nu, R)} .
$$


Since

$$
\left|w_{m}\right| \geq|w|-\left|\widetilde{w}_{m}\right|
$$

then by applying Lemma 3.3 on page 47 , we have

$$
\left|\widetilde{w}_{m}\right| \leq \frac{\left\|\widetilde{w}_{m}\right\|}{\lambda_{m+1}^{1 / 2}} \leq \frac{K_{4}(\gamma, s, v, R)}{\lambda_{m+1}^{1 / 2}},
$$

where $K_{4}(\gamma, s, \nu, R)$ is given by (3.11). From the above we reach

$$
\left|\mathcal{A}_{m}\left(u_{0}\right) w_{m}-\lambda w_{m}\right| \geq \frac{1}{K_{7}(\gamma, \rho, s, v, R)}\left(v-\frac{K_{4}(\gamma, s, v, R)}{\lambda_{m+1}^{1 / 2}}\right),
$$

where $K_{7}(\gamma, \rho, s, \nu, R)$ is as in (3.29). On the other hand,

$$
\begin{aligned}
\mathcal{A}_{m}\left(u_{0}\right) w_{m}-\lambda w_{m}= & \mathcal{A}_{m}\left(u_{0}\right) w_{m}-P_{m}\left(\mathcal{A}_{n}\left(u_{1}\right) w\right) \\
= & P_{m}\left[B\left(u_{0}-u_{1}, w_{m}\right)-B\left(u_{1}, \widetilde{w}_{m}\right)-B\left(\widetilde{w}_{m}, u_{0}\right)\right. \\
& \left.+B\left(w, u_{0}-u_{1}\right)-\gamma L \widetilde{w}_{m}\right] .
\end{aligned}
$$

By using the inequalities (2.8)-(2.10) and (2.20) with $\beta=\alpha$, we obtain

$$
\begin{aligned}
\mid\left(\mathcal{A}_{m}\right. & \left.\left(u_{0}\right)-\lambda\right) w_{m} \mid \\
\leq & C_{1}\left(\frac{\left\|u_{0}-u_{1}\right\|}{\lambda_{1}^{1 / 4}}\left\|w_{m}\right\|^{1 / 2}\left|A w_{m}\right|^{1 / 2}+\left|u_{1}\right|^{1 / 2}\left|A u_{1}\right|^{1 / 2}\left\|\widetilde{w}_{m}\right\|\right) \\
& +C_{1}\left(\left\|u_{0}\right\|^{1 / 2}\left|A u_{0}\right|^{1 / 2}\left|\widetilde{w}_{m}\right|^{1 / 2}\left\|\widetilde{w}_{m}\right\|^{1 / 2}+\left\|u_{0}-u_{1}\right\||w|^{1 / 2}|A w|^{1 / 2}\right) \\
& +\gamma v C_{L}^{1-\alpha} \lambda_{1}^{1-\alpha}\left|\widetilde{w}_{m}\right|^{1-\alpha}\left|A \widetilde{w}_{m}\right|^{\alpha} \\
\leq & C_{1} K_{5}^{1 / 2}(\gamma, s, v, R)\left(\frac{K_{4}^{1 / 2}(\gamma, s, v, R)}{\lambda_{1}^{1 / 4}}+1\right)\left\|u_{0}-u_{1}\right\| \\
& +\frac{2 C_{1} R K_{5}(\gamma, s, v, R)}{\lambda_{m+1}^{1 / 2}}+\frac{\gamma v C_{L}^{1-\alpha} \lambda_{1}^{1-\alpha} K_{5}(\gamma, s, v, R)}{\lambda_{m+1}^{1-\alpha}},
\end{aligned}
$$

where $K_{4}(\gamma, s, v, R)$ and $K_{5}(\gamma, s, v, R)$ are given by (3.11) and (3.12), respectively.

Now if we let

$$
\left\|u_{0}-u_{1}\right\| \leq \eta(\gamma, \rho, s, \nu, R) \quad \text { and } \quad m \geq M_{2}(\gamma, \rho, s, \nu, R),
$$

where $\eta(\gamma, \rho, s, \nu, R)$ and $M_{2}(\gamma, \rho, s, \nu, R)$ are as in (3.33) and (3.34), respectively, then 


$$
\begin{aligned}
\frac{v}{K_{7}(\gamma, \rho, s, v, R)} \geq C_{1} & K_{5}^{1 / 2}(\gamma, s, v, R)\left[\frac{K_{4}^{1 / 2}(\gamma, s, v, R)}{\lambda_{1}^{1 / 4}}+1\right]\left\|u_{0}-u_{1}\right\| \\
& +\frac{2 C_{1} R K_{5}(\gamma, s, \nu, R)}{\lambda_{m+1}^{1 / 2}}+\frac{K_{4}(\gamma, s, v, R)}{K_{7}(\gamma, \rho, s, \nu, R) \lambda_{m+1}^{1 / 2}} \\
& +\frac{\gamma v C_{L}^{1-\alpha} \lambda_{1}^{1-\alpha} K_{5}(\gamma, s, \nu, R)}{\lambda_{m+1}^{1-\alpha}} .
\end{aligned}
$$

This leads into a contradiction. Thus, $\lambda \in B\left(\operatorname{Sp}\left(\mathcal{A}_{m}\left(u_{0}\right)\right), \rho\right)$, i.e.,

$$
\left(\operatorname{Sp}\left(\mathcal{A}_{n}\left(u_{1}\right)\right)\right)_{s} \subset B\left(\operatorname{Sp}\left(\mathcal{A}_{m}\left(u_{0}\right)\right), \rho\right) .
$$

Since $f \in H$, then there exist a constant $M_{3}\left(\gamma, \sigma, v, f, K_{3}\right)$ such that

$$
\left|Q_{m} f\right|<\frac{\lambda_{1}}{8 C_{1} K_{9}\left(\gamma, \sigma / 2, \sigma, \nu, K_{3}\right)} \quad \forall m \geq M_{3}(\gamma, \sigma, \nu, f),
$$

where $K_{3}$ is as in (3.6) and $K_{9}\left(\sigma / 2, \sigma, v, K_{3}\right)$ is as in (3.31).

Indeed, given $f$, we can compute $K_{3}$ and $K_{9}\left(\sigma / 2, \sigma, v, K_{3}\right)$, and then estimate $M_{3}\left(\gamma, \sigma, \nu, f, K_{3}\right)$. However, we do not have a priori knowledge about the magnitude of $M_{3}\left(\gamma, \sigma, v, f, K_{3}\right)$. For the case $f \in V$, we have an explicit a priori estimate

$$
M_{3}\left(\gamma, \sigma, v, f, K_{3}\right)=\frac{\left(8 C_{1}\|f\| K_{9}\left(\gamma, \sigma / 2, \sigma, v, K_{3}\right)\right)^{2}}{C_{0} \lambda_{1}^{3}} .
$$

3.2. The main result in the Galerkin case. Next we present our main theorem of this section.

Theorem 3.2. Let $\sigma>0$ be given, and let $u_{m}^{\infty} \in V$ be a steady state solution of the Galerkin approximation (3.1) of the closed-loop NSE (2.18) with $\left|u_{m}^{\infty}\right| \leq K_{1}$. Then there exists a constant

$$
M_{\mathrm{GLK}}\left(\gamma, \sigma, \nu, f, K_{1}\right)=\max \left\{M_{4}\left(\gamma, \sigma, \nu, f, K_{1}\right), M_{5}\left(\gamma, \sigma, \nu, f, K_{1}\right)\right\},
$$

where $M_{4}\left(\gamma, \sigma, \nu, f, K_{1}\right), M_{5}\left(\gamma, \sigma, \nu, f, K_{1}\right)$ are specified in (3.49) and (3.59), respectively, such that if the condition

$$
\left(\operatorname{Sp}\left(\mathcal{A}_{m}\left(u_{m}^{\infty}\right)\right)\right)_{\sigma}=\varnothing
$$

holds for some $m \geq M_{\mathrm{GLK}}\left(\gamma, \sigma, v, f, K_{1}\right)$, then we have 
56 Chongsheng CaO, IoAnnis G. Kevrekidis \& Edriss S. Titi

(i) For every $n \in[m, \infty]$ there is a hyperbolic stationary solution $u_{n}^{\infty}$ of the Galerkin approximation equation (3.1) of order $n$, for which

$$
\begin{gathered}
\left(\operatorname{Sp}\left(\mathcal{A}\left(u_{n}^{\infty}\right)\right)\right)_{\sigma / 2}=\varnothing, \\
\left|u_{m}^{\infty}-u_{n}^{\infty}\right| \leq \frac{K_{10}\left(\gamma, \sigma, v, f, K_{1}\right)}{\lambda_{m+1}}, \\
\left|A\left(u_{m}^{\infty}-u_{n}^{\infty}\right)\right| \leq K_{11}\left(\gamma, \sigma, \nu, f, K_{1}\right),
\end{gathered}
$$

where

$$
\begin{aligned}
& K_{10}\left(\gamma, \sigma, \nu, f, K_{1}\right)=1+\frac{4 C_{1} K_{3} K_{8}\left(\gamma, \sigma / 2, \sigma, \nu, K_{3}\right)}{\lambda_{1}^{1 / 2}} \\
&+2 \gamma \nu\left(\frac{\lambda_{1} C_{L}}{\lambda_{m+1}}\right)^{1-\alpha},
\end{aligned}
$$

$$
K_{11}\left(\gamma, \sigma, v, f, K_{1}\right)=\frac{\lambda_{1} K_{9}\left(\gamma, \sigma / 2, \sigma, \nu, K_{3}\right)}{2 C_{1}},
$$

where $\left.K_{8}\left(\gamma, \sigma / 2, \sigma, \nu, K_{3}\right)\right)$ and $\left.K_{9}\left(\gamma, \sigma / 2, \sigma, v, K_{3}\right)\right)$ are as in (3.30) and (3.31), respectively.

(ii) There is a stationary solution $u^{\infty}$ of the closed-loop system (2.18) such that

$$
u_{n}^{\infty} \rightarrow u^{\infty} \text { as } n \rightarrow \infty .
$$

Moreover, $u^{\infty}$ is hyperbolic and

$$
\begin{gathered}
\left(\operatorname{Sp}\left(\mathcal{A}\left(u^{\infty}\right)\right)\right)_{\sigma / 2}=\varnothing, \\
\left|u^{\infty}-u_{n}^{\infty}\right| \leq \frac{K_{10}\left(\gamma, \sigma, v, f, K_{1}\right)}{\lambda_{n+1}} .
\end{gathered}
$$

Proof. First we will show the existence of $u_{n}^{\infty}$. Let $v=u_{n}^{\infty}-u_{m}^{\infty}$. If $u_{n}^{\infty}$ is a stationary solution of the Galerkin equations (3.1) of order $n$, then $v$ should satisfy

(3.48) $v A v+P_{n} B\left(u_{m}^{\infty}+v, u_{m}^{\infty}+v\right)-P_{m} B\left(u_{m}^{\infty}, u_{m}^{\infty}\right.$

$$
+\gamma P_{n} L\left(u_{m}^{\infty}+v\right)-\gamma P_{m} L u_{m}^{\infty}=P_{n} f-P_{m} f .
$$

Thus,

$$
\begin{aligned}
\mathcal{A}_{n}\left(u_{m}^{\infty}\right) v & =v A v+P_{n}\left[B\left(u_{m}^{\infty}, v\right)+B\left(v, u_{m}^{\infty}\right)+\gamma L v\right] \\
& =Q_{m} P_{n} f-Q_{m} P_{n} B\left(u_{m}^{\infty}, u_{m}^{\infty}\right)-P_{n} B(v, v)-\gamma Q_{m} P_{n} L u_{m}^{\infty} .
\end{aligned}
$$


By Lemma 3.7 on page 53, we have $\left(\operatorname{Sp}\left(\mathcal{A}_{n}\left(u_{m}^{\infty}\right)\right)\right)_{\sigma / 2}=\varnothing$, when $m \geq$ $M_{2}\left(\sigma, \sigma / 2, v, K_{3}\right)$. Therefore, $\mathcal{A}_{n}\left(u_{m}^{\infty}\right)$ is invertible. Denote by

$\mathcal{F}(v)=\left(\mathcal{A}_{n}\left(u_{m}^{\infty}\right)\right)^{-1}\left(Q_{m} P_{n} f-Q_{m} P_{n} B\left(u_{m}^{\infty}, u_{m}^{\infty}\right)-P_{n} B(v, v)-\gamma Q_{m} P_{n} L u_{m}^{\infty}\right)$.

By Lemma 3.6 on page 50 , we get

$$
\begin{aligned}
|A \mathcal{F}(v)| \leq & K_{9}\left(\gamma, \frac{\sigma}{2}, \sigma, K_{3}, v, f\right) \\
& \times\left|Q_{m} P_{n} f-Q_{m} P_{n} B\left(u_{m}^{\infty}, u_{m}^{\infty}\right)-P_{n} B(v, v)-\gamma Q_{m} P_{n} L u_{m}^{\infty}\right| .
\end{aligned}
$$

By using (2.9), (2.11), and (2.20) with $\beta=\alpha$, we obtain

$$
\begin{aligned}
|A \mathcal{F}(v)| \leq K_{9}\left(\gamma, \frac{\sigma}{2}, \sigma, v, K_{3}\right)\left[\left|Q_{m} f\right|\right. & +\frac{2 C_{1} K_{3}^{2}}{\lambda_{1}^{1 / 2} \lambda_{m+1}^{1 / 2}} \\
& \left.+\frac{\gamma v C_{L}^{1-\alpha} \lambda_{1}^{1-\alpha} K_{3}}{\lambda_{m+1}^{1-\alpha}}+\frac{C_{1}|A v|^{2}}{\lambda_{1}}\right] .
\end{aligned}
$$

Therefore, if $m \geq M_{4}(\gamma, \sigma, v, f)$, where

(3.49) $M_{4}\left(\gamma, \sigma, v, f, K_{1}\right)$

$$
\begin{gathered}
=\max \left\{M_{2}\left(\sigma, \frac{\sigma}{2}, \nu, K_{3}\right), M_{3}, \frac{1024 C_{1}^{4} K_{3}^{4} K_{9}^{4}\left(\gamma, \sigma / 2, \sigma, v, K_{3}\right)}{C_{0} \lambda_{1}^{4}},\right. \\
\left.\frac{C_{L}}{C_{0}}\left(\frac{16 C_{1} \gamma \nu K_{3} K_{9}^{2}\left(\gamma, \sigma / 2, \sigma, \nu, K_{3}\right)}{\lambda_{1}}\right)^{1 /(1-\alpha)}\right\},
\end{gathered}
$$

then

$$
\left|Q_{m} f\right|+\frac{2 C_{1} K_{3}^{2}}{\lambda_{1}^{1 / 2} \lambda_{m+1}^{1 / 2}}+\frac{\gamma \nu C_{L}^{1-\alpha} \lambda_{1}^{1-\alpha} K_{3}}{\lambda_{m+1}^{1-\alpha}}<\frac{\lambda_{1}}{4 C_{1} K_{9}^{2}\left(\gamma, \sigma / 2, \sigma, \nu, K_{3}\right)} .
$$

As a result, $\mathcal{F}$ maps

$$
\bar{B}_{r}=\left\{v \in H_{n}:|A v| \leq r\right\}
$$

into $\bar{B}_{r}$, where

$$
\begin{aligned}
r & =2\left[\left|Q_{m} f\right|+\frac{C_{1} K_{3}^{2}}{\lambda_{1}^{1 / 2} \lambda_{m+1}^{1 / 2}}+\frac{\gamma \nu C_{L}^{1-\alpha} \lambda_{1}^{1-\alpha} K_{3}}{\lambda_{m+1}^{1-\alpha}}\right] K_{9}\left(\gamma, \frac{\sigma}{2}, \sigma, \nu, K_{3}\right) \\
& <\frac{\lambda_{1}}{2 C_{1} K_{9}\left(\gamma, \sigma / 2, \sigma, \nu, K_{3}\right)} .
\end{aligned}
$$


Moreover,

$$
\left|A\left(\mathcal{F}\left(v_{1}\right)-\mathcal{F}\left(v_{2}\right)\right)\right| \leq \frac{2 C_{1} r K_{9}\left(\gamma, \sigma / 2, \sigma, v, K_{3}\right)}{\lambda_{1}}\left|A\left(v_{2}-v_{1}\right)\right|,
$$

and

$$
\frac{2 C_{1} r K_{9}\left(\gamma, \sigma / 2, \sigma, v, K_{3}\right)}{\lambda_{1}}<1 .
$$

Therefore, $\mathcal{F}$ is a contraction map on $\bar{B}_{r}$, given by (3.50), and as a result it has a unique $v^{\infty} \in\left\{v \in H_{n}:|A v| \leq r\right\}$ such that $u_{n}^{\infty}=u_{m}^{\infty}+v^{\infty}$ is a steady state of the Galerkin equations (3.1) of order $n$, and

$$
\left|A\left(u_{n}^{\infty}-u_{m}^{\infty}\right)\right| \leq r \leq \frac{\lambda_{1}}{2 C_{1} K_{9}\left(\gamma, \sigma / 2, \sigma, \nu, K_{3}\right)},
$$

when $m \geq M_{4}\left(\gamma, \sigma, v, f, K_{1}\right)$, and $M_{4}\left(\gamma, \sigma, v, f, K_{1}\right)$ and $K_{9}\left(\gamma, \sigma / 2, \sigma, \nu, K_{3}\right)$ are as in (3.49) and (3.31), respectively.

Next we will show the estimates (3.41). Let $v_{m}=P_{m} u_{n}^{\infty}-u_{m}^{\infty}$ and $\tilde{v}_{m}=$ $Q_{m} u_{n}^{\infty}$. Subtract the Galerkin equations (3.1) of order $m$ from the one of order $n$, to get

$$
\begin{aligned}
& v A v_{m}+P_{m}\left(B\left(u_{n}^{\infty}, u_{n}^{\infty}\right)-B\left(u_{m}^{\infty}, u_{m}^{\infty}\right)+\gamma P_{m} L\left(u_{n}^{\infty}-u_{m}^{\infty}\right)\right)=0, \\
& \left.v A \widetilde{v}_{m}+Q_{m} P_{n} B\left(u_{n}^{\infty}, u_{n}^{\infty}\right)+\gamma L u_{n}^{\infty}\right)=Q_{m} P_{n} f .
\end{aligned}
$$

As a consequence of the equation (3.53) we obtain

$$
\begin{aligned}
\mathcal{A}_{m}\left(u_{m}^{\infty}\right) & v_{m} \\
& =v A v+P_{m}\left(B\left(u_{m}^{\infty}, v_{m}\right)+B\left(v_{m}, u_{m}^{\infty}\right)+\gamma L v_{m}\right) \\
& =-P_{m}\left[B\left(v_{m}+\tilde{v}_{m}, v_{m}+\tilde{v}_{m}\right)+B\left(u_{m}^{\infty}, \tilde{v}_{m}\right)+B\left(\tilde{v}_{m}, u_{m}^{\infty}\right)+\gamma L \tilde{v}_{m}\right] \\
& =-P_{m}\left[B\left(v_{m}, v_{m}\right)+B\left(u_{n}^{\infty}, \tilde{v}_{m}\right)+B\left(\tilde{v}_{m}, u_{n}^{\infty}\right)+\gamma L \tilde{v}_{m}\right] .
\end{aligned}
$$

By using (2.6) we have for every $w_{1}, w_{2}, w_{3} \in H_{m}$,

$$
\begin{aligned}
\left|\left(\left(\mathcal{A}_{m}\left(u_{m}^{\infty}\right)\right)^{-1} B\left(w_{1}, w_{2}\right), w_{3}\right)\right| & =\left|\left(B\left(w_{1}, w_{2}\right),\left(\left(\mathcal{A}_{m}\left(u_{m}^{\infty}\right)\right)^{-1}\right)^{*} w_{3}\right)\right| \\
& =\left|\left(B\left(w_{1},\left(\left(\mathcal{A}_{m}\left(u_{m}^{\infty}\right)\right)^{-1}\right)^{*} w_{3}\right), w_{2}\right)\right| .
\end{aligned}
$$

By using (2.8) and (3.27), we get

$$
\begin{aligned}
\left|\left(\left(\mathcal{A}_{m}\left(u_{m}^{\infty}\right)\right)^{-1} B\left(w_{1}, w_{2}\right), w_{3}\right)\right| \\
\left.\quad \leq C_{1}\left|w_{1}\right|^{1 / 2}\left|A w_{1}\right|^{1 / 2} \|\left(\left(\mathcal{A}_{m}\left(u_{m}^{\infty}\right)\right)^{-1}\right)^{*} w_{3}\right) \|\left|w_{2}\right| \\
\quad \leq C_{1} K_{8}\left(\gamma, \frac{\sigma}{2}, \sigma, \nu, K_{3}\right)\left|w_{1}\right|^{1 / 2}\left|A w_{1}\right|^{1 / 2}\left|w_{2}\right|\left|w_{3}\right|,
\end{aligned}
$$


where $K_{8}\left(\gamma, \sigma / 2, \sigma, v, K_{3}\right)$ is given by (3.30). Now by using (2.9) and (3.27) in (3.55), we obtain

$$
\begin{aligned}
\left|\left(\left(\mathcal{A}_{m}\left(u_{m}^{\infty}\right)\right)^{-1} B\left(w_{1}, w_{2}\right), w_{3}\right)\right| \\
\left.\quad \leq C_{1}\left|w_{1}\right| \|\left(\left(\mathcal{A}_{m}\left(u_{m}^{\infty}\right)\right)^{-1}\right)^{*} w_{3}\right) \|\left|w_{2}\right|^{1 / 2}\left|A w_{2}\right|^{1 / 2} \\
\quad \leq C_{1} K_{8}\left(\gamma, \frac{\sigma}{2}, \sigma, \nu, K_{3}\right)\left|w_{1}\right|\left|w_{2}\right|^{1 / 2}\left|A w_{2}\right|^{1 / 2}\left|w_{3}\right| .
\end{aligned}
$$

Therefore, we reach

$$
\begin{aligned}
& \left|\left(\mathcal{A}_{m}\left(u_{m}^{\infty}\right)\right)^{-1} B\left(w_{1}, w_{2}\right)\right| \\
& \quad \leq C_{1} K_{8}\left(\gamma, \frac{\sigma}{2}, \sigma, v, K_{3}\right)\left|w_{1}\right|^{1 / 2}\left|A w_{1}\right|^{1 / 2}\left|w_{2}\right|, \\
& \left|\left(\mathcal{A}_{m}\left(u_{m}^{\infty}\right)\right)^{-1} B\left(w_{1}, w_{2}\right)\right| . \\
& \quad \leq C_{1} K_{8}\left(\gamma, \frac{\sigma}{2}, \sigma, v, K_{3}\right)\left|w_{1}\right|\left|w_{2}\right|^{1 / 2}\left|A w_{2}\right|^{1 / 2}
\end{aligned}
$$

As a result of these estimates, Lemma 3.6 on page 50, and the inequalities (2.7)(2.11) and (2.20) with $\beta=0$, we obtain

$$
\begin{aligned}
\left|v_{m}\right| \leq\left|\left(\mathcal{A}_{m}\left(u_{m}^{\infty}\right)\right)^{-1} P_{m}\left[B\left(v_{m}, v_{m}\right)+B\left(u_{n}^{\infty}, \widetilde{v}_{m}\right)+B\left(\widetilde{v}_{m}, u_{n}^{\infty}\right)+L \widetilde{v}_{m}\right]\right| \\
\leq \frac{C_{1}}{\lambda_{1}^{1 / 4}} K_{9}\left(\gamma, \frac{\sigma}{2}, \sigma, v, K_{3}\right)\left|v_{m}\right|^{3 / 2}\left\|v_{m}\right\|^{1 / 2} \\
\quad+2 C_{1} K_{8}\left(\gamma, \frac{\sigma}{2}, \sigma, v, K_{3}\right) K_{1}^{1 / 2} K_{3}^{1 / 2}\left|\tilde{v}_{m}\right| \\
+\frac{\gamma v C_{L}^{1-\alpha} \lambda_{1}^{1-\alpha} K_{9}\left(\gamma, \sigma / 2, \sigma, v, K_{3}\right)}{\lambda_{m+1}^{1-\alpha}}\left|\widetilde{v}_{m}\right| .
\end{aligned}
$$

Notice that

$$
\left|v_{m}\right|^{1 / 2}\left\|v_{m}\right\|^{1 / 2} \leq \frac{\left|A v_{m}\right|}{\lambda_{1}^{3 / 4}}
$$

and

$$
\frac{C_{1}}{\lambda_{1}} K_{9}\left(\gamma, \frac{\sigma}{2}, \sigma, \nu, K_{3}\right)\left|A v_{m}\right| \leq \frac{1}{2} .
$$

Therefore, by using the estimates (3.52), we get

$$
\left|v_{m}\right| \leq \frac{K_{12}\left(\gamma, \sigma, v, f, K_{1}\right)}{\lambda_{m+1}},
$$


where

$$
K_{12}\left(\gamma, \sigma, v, f, K_{1}\right)=\frac{4 C_{1} K_{3} K_{8}\left(\gamma, \sigma / 2, \sigma, \nu, K_{3}\right)}{\lambda_{1}^{1 / 2}}+2 \gamma \nu\left(\frac{\lambda_{1} C_{L}}{\lambda_{m+1}}\right)^{1-\alpha} .
$$

Then the estimate (3.41) is achieved.

Let

$$
M_{5}\left(\gamma, \sigma, \nu, f, K_{1}\right)=\frac{K_{12}\left(\gamma, \sigma, \nu, f, K_{1}\right)}{C_{0} \lambda_{1} \eta\left(\gamma, \sigma, \sigma / 2, \nu, K_{3}\right)} .
$$

We apply Lemma 3.7 on page 53 , when $m \geq M_{\mathrm{GLK}}\left(\gamma, \sigma, v, f, K_{1}\right)$, to conclude

$$
\left(\operatorname{Sp}\left(\mathcal{A}\left(u^{\infty}\right)\right)\right)_{\sigma / 2}=\varnothing .
$$

\section{A STABILIZATION CRITERION FOR THE NONLINEAR GALERKIN APPROXIMATION}

Without loss of generality, we will assume that $\alpha=\frac{1}{2}$ in the condition (2.20) in this section. By now, it is a well established fact that, for the dissipative parabolic evolution equations, the nonlinear Galerkin method, which is based on the theory of Inertial Manifolds [32] and Approximate Inertial Manifolds (AIM) (see, e.g., [27], [33], [53], [63], [76] and references therein), is a more accurate numerical method than the standard Galerkin method (cf. [23], [39], [47], [48]). Motivated by this superiority in accuracy of the nonlinear Galerkin method, we show here that a similar criterion, yet stronger than the one stated in the previous section, is available in the context of nonlinear Galerkin method.

Here, we will restrict ourselves to the nonlinear Galerkin method that is based on one of the simplest AIM, the one introduced in [27]. That is, for the closedloop NSE (2.18) and (2.19) we have the following nonlinear Galerkin approximation:

$$
\begin{gathered}
\frac{d p_{m}}{d t}+v A p_{m}+P_{m}\left[B\left(p_{m}+q_{m}, p_{m}+q_{m}\right)-B\left(q_{m}, q_{m}\right)\right. \\
\left.+\gamma L\left(p_{m}+q_{m}\right)\right]=P_{m} f \\
v A q_{m}+Q_{m}\left[B\left(p_{m}, p_{m}\right)+\gamma L p_{m}\right]=Q_{m} f, \\
p_{m}(0)=P_{m} u_{i n},
\end{gathered}
$$

where $p_{m} \in H_{m}$ and $q_{m} \in Q_{m}$.

For $1 \leq m \leq \infty$ we denote by $p_{m}^{\infty}+q_{m}^{\infty}$ the stationary solutions of the equations (4.1) and (4.2). Specially, $q_{\infty}^{\infty}=0$ and $u^{\infty}=p_{\infty}^{\infty}$ is a steady state of the equation (2.18). We will denote by

$$
\Phi_{\text {app }}\left(u_{m}\right)=(\nu A)^{-1}\left[Q_{m} f-Q_{m} B\left(u_{m}, u_{m}\right)-\gamma Q_{m} L u_{m}\right],
$$


for any $u_{m} \in H_{m}$, the Foias-Manley-Temam AIM [27]. Furthermore, we also denote by $\mathcal{N}_{m}\left(u_{m}\right): H_{m} \rightarrow H_{m}$ the linearized operator of the nonlinear Galerkin closed-loop operator given in (1.1) and (4.2) about $u_{m}+\Phi_{\text {app }}\left(u_{m}\right)$, defined as

$$
\begin{aligned}
& \text { 4.5) } \mathcal{N}_{m}\left(u_{m}\right) w=v A w+P_{m}\left[B\left(u_{m}+\Phi_{\text {app }}\left(u_{m}\right), w\right)\right. \\
& \left.+B\left(w, u_{m}+\Phi_{\text {app }}\left(u_{m}\right)\right)+B\left(u_{m}, \xi(w)\right)+B\left(\xi(w), u_{m}\right)+\gamma L(w+\xi(w))\right],
\end{aligned}
$$

for all $w \in H_{m}$, where $\xi(w) \in Q_{m}$ satisfies

$$
v A \xi+Q_{m}\left[B\left(u_{m}, w\right)+B\left(w, u_{m}\right)+\gamma L w\right]=0 .
$$

When $m=\infty, \mathcal{N}_{\infty}\left(u_{\infty}\right)=\mathcal{A}\left(u_{\infty}\right)$.

Lemma 4.1. For any $m \in[1, \infty]$, if there is a steady state $p_{m}^{\infty}+q_{m}^{\infty} \in V$ of equations (4.1) and (4.2) with

$$
\left|p_{m}^{\infty}+q_{m}^{\infty}\right| \leq N_{1},
$$

then $\left(p_{m}^{\infty}+q_{m}^{\infty}\right) \in \mathcal{D}(A)$, and

$$
\begin{gathered}
\left\|p_{m}^{\infty}+q_{m}^{\infty}\right\| \leq N_{2}=\left(2 v \lambda_{1} G N_{1}\right)^{1 / 2}+2 \gamma C_{L}^{1 / 2} \lambda_{1}^{1 / 2} N_{1}, \\
\left|A\left(p_{m}^{\infty}+q_{m}^{\infty}\right)\right| \leq \lambda_{1}^{1 / 2} N_{3}=4 v \lambda_{1} G+\frac{12 C_{1}^{2}}{v^{2}} N_{1} N_{2}^{2}+8 \gamma C_{L}^{1 / 2} \lambda_{1}^{1 / 2} N_{2},
\end{gathered}
$$

where again $G=|f| /\left(\nu^{2} \lambda_{1}\right)$ is the Grashoff number.

Proof. Let $p_{m}^{\infty}+q_{m}^{\infty}$ be a stationary solution of the equations (4.1) and (4.2), with $\left|p_{m}^{\infty}+q_{m}^{\infty}\right| \leq N_{1}$. Taking the inner product in $H_{m}$ of the equation (4.1) with $p_{m}^{\infty}$ and the equation (4.2) with $q_{m}^{\infty}$ in $Q_{m}$, and using (2.6), we get

$$
\begin{array}{r}
v\left\|p_{m}^{\infty}\right\|^{2}+\left(B\left(p_{m}^{\infty}, q_{m}^{\infty}\right), p_{m}^{\infty}\right)+\gamma\left(L\left(p_{m}^{\infty}+q_{m}^{\infty}\right), p_{m}^{\infty}\right)=\left(f, p_{m}^{\infty}\right), \\
v\left\|q_{m}^{\infty}\right\|^{2}+\left(B\left(p_{m}^{\infty}, p_{m}^{\infty}\right), q_{m}^{\infty}\right)+\gamma\left(L\left(p_{m}^{\infty}, q_{m}^{\infty}\right)\right)=\left(f, q_{m}^{\infty}\right) .
\end{array}
$$

Adding the above equations up and using (2.6) again, we obtain

$$
v\left\|p_{m}^{\infty}\right\|^{2}+v\left\|q_{m}^{\infty}\right\|^{2}=\left(f, p_{m}^{\infty}+q_{m}^{\infty}\right)-\gamma\left(L\left(p_{m}^{\infty}+q_{m}^{\infty}\right), p_{m}^{\infty}+q_{m}^{\infty}\right)+\gamma\left(L q_{m}^{\infty}, q_{m}^{\infty}\right) .
$$

By using (2.21), we obtain

$$
v\left\|p_{m}^{\infty}\right\|^{2}+v\left\|q_{m}^{\infty}\right\|^{2} \leq|f|\left|p_{m}^{\infty}+q_{m}^{\infty}\right|+2 \gamma v C_{L}^{1 / 2} \lambda_{1}^{1 / 2}\left|p_{m}^{\infty}+q_{m}^{\infty}\right|\left\|p_{m}^{\infty}+q_{m}^{\infty}\right\| .
$$

By the Cauchy-Schwarz inequality, we reach

$$
\frac{v}{2}\left(\left\|p_{m}^{\infty}\right\|^{2}+\left\|q_{m}^{\infty}\right\|^{2}\right) \leq|f| N_{1}+2 \gamma^{2} v C_{L} \lambda_{1} N_{1}^{2},
$$


and the estimate (4.8) follows. Taking the $L^{2}$-norm of the equations (4.1) and (4.2) leads to

$$
\begin{aligned}
v\left|A\left(p_{m}^{\infty}+q_{m}^{\infty}\right)\right| \leq|f|+\left|B\left(p_{m}^{\infty}, p_{m}^{\infty}\right)\right| & +\left|B\left(p_{m}^{\infty}, q_{m}^{\infty}\right)\right| \\
& +\left|B\left(q_{m}^{\infty}, p_{m}^{\infty}\right)\right|+\gamma\left[\left|L p_{m}^{\infty}\right|+\left|L q_{m}^{\infty}\right|\right] .
\end{aligned}
$$

By using (2.7)-(2.10) and (2.20) with $\beta=\frac{1}{2}$, we conclude

$$
\begin{aligned}
v\left|A\left(p_{m}^{\infty}+q_{m}^{\infty}\right)\right| \leq|f| & +C_{1}\left|p_{m}^{\infty}\right|^{1 / 2}\left\|p_{m}^{\infty}\right\|\left|A p_{m}^{\infty}\right|^{1 / 2}+C_{1}\left|p_{m}^{\infty}\right|^{1 / 2}\left\|q_{m}^{\infty}\right\|\left|A p_{m}^{\infty}\right|^{1 / 2} \\
& +C_{1}\left|q_{m}^{\infty}\right|^{1 / 2}\left\|p_{m}^{\infty}\right\|\left|A q_{m}^{\infty}\right|^{1 / 2}+\gamma \nu C_{L}^{1 / 2} \lambda_{1}^{1 / 2}\left[\left\|p_{m}^{\infty}\right\|+\left\|q_{m}^{\infty}\right\|\right] .
\end{aligned}
$$

Thanks to the Cauchy-Schwarz inequality, we get

$$
\begin{aligned}
\frac{v}{4}\left|A\left(p_{m}^{\infty}+q_{m}^{\infty}\right)\right| \leq|f|+\frac{C_{1}^{2}}{v}\left[\left|p_{m}^{\infty}\right|\left\|p_{m}^{\infty}\right\|^{2}+p_{m}^{\infty} \mid\right. & \left.\left\|q_{m}^{\infty}\right\|^{2}+\left|q_{m}^{\infty}\right|\left\|p_{m}^{\infty}\right\|^{2}\right] \\
& +\gamma v C_{L}^{1 / 2} \lambda_{1}^{1 / 2}\left[\left\|p_{m}^{\infty}\right\|+\left\|q_{m}^{\infty}\right\|\right],
\end{aligned}
$$

which implies the estimate (4.9).

Lemma 4.2. Let $u_{m} \in H_{m}$ with $\left|A u_{m}\right| \leq \lambda_{1}^{1 / 2} R$. Then

$$
\left|A \Phi_{\mathrm{app}}\left(u_{m}\right)\right| \leq \lambda_{1}^{1 / 2} N_{4}(\gamma, R, v, f),
$$

where

$$
N_{4}(\gamma, R, v, f)=\frac{\left|Q_{m} f\right|}{v \lambda_{1}^{1 / 2}}+\frac{2 C_{1} R^{2}}{v \lambda_{m+1}^{1 / 2}}+\frac{C_{L}^{1 / 2} v \lambda_{1} R}{\lambda_{m+1}^{1 / 2}} .
$$

Suppose that $\omega \in V$ and $\xi(\omega)$ satisfies (4.6). Then

$$
\|\xi(\omega)\| \leq \frac{2 C_{1} R}{v}|\omega|+\gamma C_{L}^{1 / 2} \lambda_{1}^{1 / 2}|\omega| .
$$

Proof. Let $v_{m}=\Phi_{\text {app }}\left(u_{m}\right)$. Then $v_{m}$ satisfies

$$
v A v_{m}+Q_{m}\left[B\left(u_{m}, u_{m}\right)+\gamma L u_{m}\right]=Q_{m} f .
$$

Thus

$$
\left|A v_{m}\right| \leq \frac{1}{v}\left[\left|Q_{m} f\right|+\frac{\left|\nabla B\left(u_{m}, u_{m}\right)\right|}{v \lambda_{m+1}^{1 / 2}}+\gamma \frac{\left|A^{1 / 2} L u_{m}\right|}{\lambda_{m+1}^{1 / 2}}\right] .
$$


By using (2.11) and (2.20) with $\beta=1$, we get

$$
\left|A v_{m}\right| \leq \frac{\left|Q_{m} f\right|}{v}+\frac{2 C_{1}\left|A u_{m}\right|^{2}}{v \lambda_{1}^{1 / 2} \lambda_{m+1}^{1 / 2}}+\frac{C_{L}^{1 / 2} v \lambda_{1} R}{\lambda_{m+1}^{1 / 2}} .
$$

Then (4.10) follows. Now, taking the inner product in $H$ of equation (4.6) with $\omega$, we get

$$
v\|\xi(\omega)\|^{2} \leq\left|\left(B\left(u_{m}, \omega\right), \xi(\omega)\right)\right|+\left|\left(B\left(u_{m}, \omega\right), \xi(\omega)\right)\right|+\gamma|(L \omega, \xi(\omega))| .
$$

By using (2.7)-(2.10) and (2.21), we obtain

$$
\nu\|\xi(\omega)\|^{2} \leq 2 C_{1}\left|u_{m}\right|^{1 / 2}\left|A u_{m}\right|^{1 / 2}\|\xi(\omega)\||\omega|+\gamma \nu C_{L}^{1 / 2} \lambda_{1}^{1 / 2}\|\xi(\omega)\||\omega| .
$$

Therefore, (4.12) holds.

Lemma 4.3. Let $u_{m} \in H_{m}$ with $\left|A u_{m}\right| \leq \lambda_{1}^{1 / 2} R$. If $w$ is an eigenfunction of $\mathcal{N}_{m}\left(u_{m}\right)$, then $w \in \mathcal{D}(A)$. Moreover, if $|w|=v$ and $\mathcal{N}_{m}\left(u_{m}\right) w=\Lambda w$ for some $\Lambda$, then we have

$$
\begin{gathered}
\|w\| \leq N_{5}(\gamma, \Lambda, R, v, f), \\
|A w| \leq N_{6}(\gamma, \Lambda, R, v, f),
\end{gathered}
$$

where

$$
\begin{array}{ll}
\text { (4.15) } & N_{5}(\gamma, \Lambda, R, \nu, f) \\
= & (4|\Lambda| \nu)^{1 / 2}+2 C_{1}\left(R+N_{4}(\gamma, R, v, f)\right)+\frac{C_{1}^{2} \lambda_{1}^{1 / 2} R^{2}}{\nu \lambda_{m+1}^{1 / 2}}+\sqrt{12} \gamma \nu C_{L}^{1 / 2} \lambda_{1}^{1 / 2}, \\
(4.16) \quad & N_{6}(\gamma, \Lambda, R, v, f) \\
= & |\Lambda|+\gamma C_{L}^{1 / 2} \lambda_{1}^{1 / 2}\left[N_{5}^{1 / 2}(\gamma, s, R, v, f)+2 C_{1} R+\gamma v C_{L}^{1 / 2} \lambda_{1}^{1 / 2}\right] \\
& +\frac{2 C_{1}}{v}\left[R+N_{4}(\gamma, R, v, f)\right]\left[N_{5}(\gamma, \Lambda, R, v, f)+2 C_{1} R+\gamma \nu C_{L}^{1 / 2} \lambda_{1}^{1 / 2}\right] .
\end{array}
$$

Proof. Let $v_{m}=\Phi_{\text {app }}\left(u_{m}\right)$, and let $w$ be an eigenfunction of $\mathcal{N}_{m}\left(u_{m}\right)$ with corresponding eigenvalue $\Lambda$ such that $|w|=v$, i.e.,

$$
\begin{gathered}
v A w+P_{m}\left[B\left(u_{m}+v_{m}, w\right)+B\left(w, u_{m}+v_{m}\right)+B\left(u_{m}, \xi(w)\right)\right. \\
\left.+B\left(\xi(w), u_{m}\right)+\gamma L(w+\xi(w))\right]=\Lambda w \\
v A \xi+Q_{m}\left(B\left(u_{m}, w\right)+B\left(w, u_{m}\right)+\gamma L w\right)=0 .
\end{gathered}
$$


Taking the inner product in $H$ of the equations above with $w$ and $\xi$ respectively, gives

$$
\begin{gathered}
v\|w\|^{2}+\left(B\left(w, u_{m}+v_{m}\right), w\right)+\left(B\left(u_{m}, \xi\right), w\right) \\
+\left(B\left(\xi, u_{m}\right), w\right)+\gamma(L(w+\xi), w)=\Lambda|w|^{2}, \\
v\|\xi\|^{2}+\left(B\left(u_{m}, w\right), \xi\right)+\left(B\left(w, u_{m}\right), \xi\right)+\gamma(L w, \xi)=0 .
\end{gathered}
$$

Adding up these two equations,

$$
\begin{aligned}
v\|w\|^{2}+v\|\xi\|^{2} \leq & |\Lambda||w|^{2}+\left|\left(B\left(w, u_{m}+v_{m}\right), w\right)\right|+\left|\left(B\left(\xi(w), u_{m}\right), w\right)\right| \\
& +\left|\left(B\left(w, u_{m}\right), \xi\right)\right|+\gamma|(L w, w)|+\gamma|(L w, \xi)|+\gamma|(L \xi, w)| .
\end{aligned}
$$

By using (2.7)-(2.10) and (2.21), we obtain

$$
\begin{aligned}
v\|w\|^{2}+v\|\xi\|^{2} \leq|\Lambda| & |w|^{2}+C_{1}\left\|u_{m}+v_{m}\right\||w|\|w\| \\
& +\frac{2 C_{1}}{\lambda_{1}^{1 / 2}}\left|A u_{m}\right||w|^{1 / 2}\|w\|^{1 / 2}|\xi|+\gamma \nu C_{L}^{1 / 2} \lambda_{1}^{1 / 2}|w|\|w\| \\
& +2 \gamma \nu C_{L}^{1 / 2} \lambda_{1}^{1 / 2}|w|\|\xi\| .
\end{aligned}
$$

By Young's inequality and the Cauchy-Schwarz inequality, we reach

$$
\frac{v}{2}\|w\|^{2} \leq v^{2}|\Lambda|+\frac{C_{1}^{4} \lambda_{1}^{2} R^{4}}{4 v \lambda_{m+1}^{2}}+v C_{1}^{2}\left[R+N_{4}(\gamma, R, v, f)\right]^{2}+3 \gamma^{2} v^{3} C_{L} \lambda_{1} .
$$

Therefore, the estimate (3.9) is proved. Moreover, by taking the $L^{2}$-norm of the equation (4.17), we get

$$
\begin{aligned}
v|A w| \leq v|\Lambda|+\mid B\left(u_{m}+v_{m}, w\right) & +B\left(w, u_{m}+v_{m}\right) \\
& +B\left(u_{m}, \xi\right)+B\left(\xi, u_{m}\right)+\gamma L(w+\xi) \mid .
\end{aligned}
$$

By using (2.7)-(2.10) and (2.21) again to the above estimate and equation (4.20), we have

$$
\begin{aligned}
v|A w| \leq v \mid & \left.\left|+\frac{C_{1}}{\lambda_{1}^{1 / 2}}\right| A\left(u_{m}+v_{m}\right)\left|\|w\|+\frac{C_{1}}{\lambda_{1}^{1 / 4}}\right| w\right|^{1 / 2}\|w\|^{1 / 2}\left|A\left(u_{m}+v_{m}\right)\right| \\
& +\frac{C_{1}}{\lambda_{1}^{1 / 2}}\left|A\left(u_{m}+v_{m}\right)\right|\|\xi\|+\frac{C_{1}}{\lambda_{1}^{1 / 4} \lambda_{m+1}^{1 / 4}}\|\xi\|\left|A\left(u_{m}+v_{m}\right)\right| \\
& +\gamma \nu C_{L}^{1 / 2} \lambda_{1}^{1 / 2}\|w+\xi\| .
\end{aligned}
$$


From the estimate (4.12), we obtain

$$
\|\xi\| \leq 2 C_{1} R+\gamma \nu C_{L}^{1 / 2} \lambda_{1}^{1 / 2} .
$$

As a result of these estimates we get (4.14), which completes the proof of the lemma.

Lemma 4.4. Let $R>0, u_{m} \in \mathcal{D}(A)$ with $\left|A u_{m}\right| \leq \lambda_{1}^{1 / 2} R$, and $s \in \mathbb{R}$, and let $T_{1}(s)$ be the number of the eigenvalues of $\mathcal{N}_{m}\left(u_{m}\right)$ whose real parts are less than or equal to $s$, i.e.,

$$
T_{1}(s)=\# \text { of }\left\{\lambda \in \operatorname{Sp}\left(\mathcal{N}_{m}\left(u_{m}\right)\right): \mathfrak{R} \lambda<s\right\} .
$$

Then

$$
T_{1}(s) \leq N_{7}(\gamma, s, R, v, f),
$$

where

$$
\begin{array}{r}
N_{7}(\gamma, s, R, v, f)=\frac{1}{2}\left\{\left(\frac{4 s C_{0}}{v \lambda_{1}}+\frac{8 C_{1}^{2} R^{2}}{v^{2} \lambda_{1}^{1 / 2} \lambda_{m+1}^{1 / 4}}+12 \gamma^{2} C_{L} C_{0}-1\right)\right. \\
+\left[\left(\frac{4 s C_{0}}{v \lambda_{1}}+\frac{8 C_{1}^{2} R^{2}}{v^{2} \lambda_{1}^{1 / 2} \lambda_{m+1}^{1 / 4}}+12 \gamma^{2} C_{L} C_{0}-1\right)^{2}\right. \\
\left.\left.+\frac{16 C_{0} C_{2}\left(R+N_{4}(\gamma, R, v, f)\right)^{2}}{v^{2} \lambda_{1}}\right]^{1 / 2}\right\} .
\end{array}
$$

Remark. Here again, in a sense, the estimate (4.21) is sharp since the constant $N_{7}(\gamma, s, R, v, f)$ is growing linearly in $s$. For example, when $u_{\text {in }}=0$ and $\gamma=0$ we have $\mathcal{A}\left(u_{\text {in }}\right)=v A$, and in this case the $\Lambda_{j}=\lambda_{j}$ behaves like $j$ asymptotically (see (2.5)). Indeed, $T_{1}(s) \sim s$ in this special case.

Proof. Let $m<\infty$ be finite, and let $\Lambda_{1}, \ldots, \Lambda_{K}$ be the eigenvalues of $\mathcal{N}_{m}\left(u_{m}\right)$ repeated according to their multiplicities such that $\Re \Lambda_{j} \leq s$ for $j=1,2, \ldots, K$. We will denote by $E_{K}$ the linear space spanned by the generalized eigenfunctions of $\mathcal{N}_{m}\left(u_{m}\right)$ corresponding the the eigenvalues $\Lambda_{1}, \ldots, \Lambda_{K}$. Let $\psi_{1}, \ldots, \psi_{K}$ be an orthogonal basis of $E_{K}$ with $\left|\psi_{j}\right|=\nu$ for $j=1,2, \ldots, K$, and let $\Pi_{K}: H_{m} \rightarrow E_{K}$ be the orthogonal projection. We set $v_{m}=\Phi_{\text {app }}\left(u_{m}\right)$ and observe that 
66 Chongsheng CaO, IoAnnis G. Kevrekidis \& Edriss S. Titi

$$
\begin{aligned}
\mathfrak{R}\left(\sum_{j=1} K \Lambda_{j}\right)= & \mathfrak{R}\left\{\operatorname{trace}\left(\Pi_{K} \mathcal{N}_{m}\left(u_{m}\right) \Pi_{K}\right)\right\} \\
= & v^{-2} \mathfrak{R}\left(\sum_{j=1} K\left(\mathcal{N}_{m}\left(u_{m}\right) \psi_{j}, \psi_{j}\right)\right) \\
= & v^{-2} \mathfrak{R}\left\{\sum _ { j = 1 } K \left[v\left(A \psi_{j}, \psi_{j}\right)+\left(B\left(\psi_{j}, u_{m}+v_{m}\right), \psi_{j}\right)\right.\right. \\
& +\left(B\left(u_{m}, \xi\left(\psi_{j}\right)\right), \psi_{j}\right)+\left(B\left(\xi\left(\psi_{j}\right), u_{m}\right), \psi_{j}\right) \\
& \left.+\gamma\left(L\left(\psi_{j}+\xi\left(\psi_{j}\right), \psi_{j}\right)\right]\right\} .
\end{aligned}
$$

Since

$$
v A \xi\left(\psi_{j}\right)+Q_{m}\left[B\left(u_{m}, \psi_{j}\right)+B\left(\psi_{j}, u_{m}\right)+\gamma L \psi_{j}\right]=0,
$$

by taking the inner product in $H$ of the above equation with $\xi\left(\psi_{j}\right)$, and by using (2.6), we have

$$
\begin{aligned}
\left(B\left(u_{m}, \xi\left(\psi_{j}\right)\right), \psi_{j}\right)=v\left(A \xi\left(\psi_{j}\right), \xi\left(\psi_{j}\right)\right)+\left(B\left(\psi_{j}, u_{m}\right)\right. & \left., \xi\left(\psi_{j}\right)\right) \\
& +\gamma\left(L \psi_{j}, \xi\left(\psi_{j}\right)\right) .
\end{aligned}
$$

Substituting $\left(B\left(u_{m}, \xi\left(\psi_{j}\right), \psi_{j}\right)\right)$ in (4.23) gives us

$$
\begin{aligned}
\mathfrak{R}\left(\sum_{j=1} K \Lambda_{j}\right)=v^{-2} \mathfrak{R}\left\{\sum _ { j = 1 } K \left[v\left(A \psi_{j}, \psi_{j}\right)+v\left(A \xi\left(\psi_{j}\right), \xi\left(\psi_{j}\right)\right)+\gamma\left(L \psi_{j}, \xi\left(\psi_{j}\right)\right)\right.\right. \\
+\left(B\left(\xi\left(\psi_{j}\right), u_{m}\right), \psi_{j}\right)+\left(B\left(\psi_{j}, u_{m}\right), \xi\left(\psi_{j}\right)\right) \\
\left.\left.+\left(B\left(\psi_{j}, u_{m}+v_{m}\right), \psi_{j}\right)+\gamma\left(L\left(\psi_{j}+\xi\left(\psi_{j}\right)\right), \psi_{j}\right)\right]\right\} .
\end{aligned}
$$

By (2.21), we obtain

$$
\begin{aligned}
& \mathfrak{R}\left(\sum_{j=1} K \Lambda_{j}\right) \geq v^{-2} \sum_{j=1} K\left[\nu\left\|\psi_{j}\right\|^{2}+v\left\|\xi\left(\psi_{j}\right)\right\|^{2}-\left|\left(B\left(\psi_{j}, u_{m}+v_{m}\right), \psi_{j}\right)\right|\right. \\
&-\left|\left(B\left(\xi\left(\psi_{j}\right), u_{m}\right), \psi_{j}\right)\right|-\left|\left(B\left(\psi_{j}, u_{m}\right), \xi\left(\psi_{j}\right)\right)\right| \\
&\left.-\gamma \nu C_{L}^{1 / 2} \lambda_{1}^{1 / 2}\left|\psi_{j}\right|\left(\left\|\psi_{j}\right\|+2\left\|\xi\left(\psi_{j}\right)\right\|\right)\right] .
\end{aligned}
$$


Now let us estimate each term in the right hand side of the above inequality. First by the Cauchy-Schwarz inequality and Lieb-Thirring inequality (2.12), we reach

$$
\begin{aligned}
\sum_{j=1} K\left|\left(B\left(\psi_{j}, u_{m}+v_{m}\right), \psi_{j}\right)\right| & \leq\left(\int_{\Omega}\left(\sum_{j=1} K \psi_{j}(x) \cdot \psi_{j}(x)\right) d x\right)^{1 / 2}\left\|u_{m}+v_{m}\right\| \\
& \leq\left(C_{2} v^{2} \sum_{j=1} K\left\|\psi_{j}\right\|^{2}\right)^{1 / 2}\left\|u_{m}+v_{m}\right\| \\
& \leq \frac{v}{4} \sum_{j=1} K\left\|\psi_{j}\right\|^{2}+C_{2}\left\|u_{m}+v_{m}\right\|^{2} .
\end{aligned}
$$

By using (2.7)-(2.10), we obtain

$$
\left|\left(B\left(\xi\left(\psi_{j}\right), u_{m}\right), \psi_{j}\right)\right| \leq C_{1} R\left|\xi\left(\psi_{j}\right)\right|\left\|\xi\left(\psi_{j}\right)\right\|,
$$

and

$$
\left|\left(B\left(\psi_{j}, u_{m}\right), \xi\left(\psi_{j}\right)\right)\right| \leq C_{1} R\left|\xi\left(\psi_{j}\right)\right|\left\|\xi\left(\psi_{j}\right)\right\| .
$$

Thanks to the Cauchy-Schwarz inequality, we get

$$
\begin{aligned}
\gamma \nu C_{L}^{1 / 2} \lambda_{1}^{1 / 2}\left|\psi_{j}\right| & \left(\left\|\psi_{j}\right\|+2\left\|\xi\left(\psi_{j}\right)\right\|\right) \\
& \left.\leq \frac{v}{4}\left\|\psi_{j}\right\|^{2}+\gamma^{2} v C_{L} \lambda_{1}\left|\psi_{j}\right|^{2}+2 \gamma \nu C_{L}^{1 / 2} \lambda_{1}^{1 / 2}\right]\left|\psi_{j}\right|\left\|\xi\left(\psi_{j}\right)\right\| .
\end{aligned}
$$

On the other hand,

$$
\begin{gathered}
\sum_{j=1} K\left\|\psi_{j}\right\|^{2}=\sum_{j=1} K\left(A \psi_{j}, \psi_{j}\right) \geq \sum_{j=1} K \nu^{2} \lambda_{j}, \\
K s \geq \mathfrak{R}\left(\sum_{j=1} K \Lambda_{j}\right) .
\end{gathered}
$$

Therefore, by applying (4.12), we get

$$
K s \geq \mathfrak{R}\left(\sum_{j=1} K \Lambda_{j}\right) \geq \sum_{j=1} K\left[\frac{v}{2} \lambda_{j}-3 v \gamma^{2} C_{L} \lambda_{1}-\frac{2 C_{1}^{2} \lambda_{1}^{1 / 2} R^{2}}{v \lambda_{m+1}^{1 / 2}}\right]-\frac{C_{2}\left\|u_{m}+v_{m}\right\|^{2}}{v} .
$$

By using (2.5) we reach

$$
K s \geq \frac{v \lambda_{1}}{2 C_{0}} \frac{K(K+1)}{2}-3 v \gamma^{2} C_{L} \lambda_{1} K-\frac{2 C_{1}^{2} \lambda_{1}^{1 / 2} R^{2}}{\lambda_{m+1}^{1 / 2}} K-\frac{C_{2}}{v}\left(R+N_{4}(\gamma, R, v, f)\right),
$$

which implies (4.21). 
4.1. Estimates for the resolvents in the nonlinear Galerkin case. In this section we define as in Prodi [67] the parabola-like set $\tau(R) \subset \mathbb{C}$, by setting

$$
\begin{aligned}
& \tau(R)=\{z \in \mathbb{C}: \exists x \geq \lambda_{1},|z-v x| \\
& \leq\left[C_{1}(R+\varphi(R))+\gamma v \lambda_{1}^{1 / 2} C_{L}^{1 / 2}\right] x^{1 / 2} \\
&\left.+\left[\frac{3 C_{1} R}{2 \lambda_{1}^{1 / 4} \lambda_{m+1}^{1 / 4}}+\frac{\gamma v \lambda_{1}^{1 / 2} C_{L}^{1 / 2}}{\lambda_{m+1}^{1 / 2}}\right] x\right\},
\end{aligned}
$$

where $C_{1}$ and $C_{L}$ are as in (2.7) and (2.21) respectively, and

$$
\zeta(R)=\frac{|f|}{v \lambda_{m+1}^{1 / 2}}+\frac{C_{1} R^{2}}{v \lambda_{m+1}^{1 / 4}}+\frac{\gamma \lambda_{1}^{1 / 2} C_{L}^{1 / 2}}{\lambda_{m+1}^{1 / 2}} .
$$

Denote by

$$
\bar{M}=\max \left\{\frac{1}{C_{0} \lambda_{1}^{2}}\left(\frac{6 C_{1} R}{v}\right)^{4}, \frac{16 \gamma^{2} C_{L}}{C_{0}}\right\} .
$$

It is clear that when $m \geq \bar{M}, \tau(R)$ is a parabola-like set. Now and later we assume that $m \geq \bar{M}$.

Similar to Section 3 on page 44, we can show the following two lemmas.

Lemma 4.5. Let $R>0, u_{m} \in V$ with $\left\|u_{m}\right\| \leq R$. Then

- the spectra of $\mathcal{N}_{m}\left(u_{m}\right), m=1, \ldots, \infty$, are in $\tau(R)$;

- for every $z \notin T(R)$ the following inequalities hold:

$$
\begin{aligned}
\left|\left(\mathcal{N}_{m}\left(u_{m}\right)-z\right)^{-1} v\right| \leq \operatorname{dist}(z, \tau(R))^{-1}|v|= & N_{H}(\gamma, R, v, f, z)|v|, \\
\left\|\left(\mathcal{A}_{m}\left(u_{0}\right)-z\right)^{-1} v\right\| \leq N_{V}(\gamma, v, R, z)|v|, & \forall v \in H_{m}, \\
\left|A\left(\mathcal{A}_{m}\left(u_{0}\right)-z\right)^{-1} v\right| \leq N_{A}(\gamma, v, R, z)|v|, & \forall v \in H_{m},
\end{aligned}
$$


Numerical Criterion for the Stabilization of Steady States of the NSE

(4.29) $\quad N_{V}(\gamma, R, v, f, z)$

$$
\begin{gathered}
=\left(\frac{2 N_{H}(\gamma, R, v, f, z)}{v}\right)^{1 / 2}+\left(\frac{2|z|}{v}\right)^{1 / 2} N_{H}(\gamma, R, v, f, z) \\
+\left[\frac{\sqrt{2} C_{1}\left(R+N_{4}(\gamma, R, v, f)\right)}{v}+\gamma \sqrt{3 C_{L} \lambda_{1}}+\frac{2 C_{1} \lambda_{1}^{1 / 4} R}{v \lambda_{m+1}^{1 / 4}}\right] \\
\times N_{H}(\gamma, R, v, f, z),
\end{gathered}
$$

(4.30) $\quad N_{A}(\gamma, R, v, f, z)$

$$
\begin{aligned}
=\frac{1}{v}+ & \left(\frac{2 C_{1} R}{v}+\gamma C_{L}^{1 / 2} \lambda_{1}^{1 / 2}\right)^{2} N_{H}(\gamma, R, v, f, z) \\
+ & {\left[\left(\frac{2|z|}{v}\right)^{1 / 2}+\frac{2 C_{1}}{v}\left(R+N_{4}(\gamma, R, v, f)\right)+\gamma C_{L}^{1 / 2} \lambda_{1}^{1 / 2}\right] } \\
& \times N_{V}(\gamma, R, v, f, z),
\end{aligned}
$$

Lemma 4.6. Let $R>0, u_{m} \in V$ with $\left\|u_{m}\right\| \leq R$. Then for every $z \notin \tau(R)$ the operators $\left(\mathcal{N}_{m}\left(u_{m}\right)-z\right)^{-1}$, for $m=1, \ldots, \infty$, are H-S operators. Moreover,

$$
\left\|\left(\mathcal{N}_{m}\left(u_{m}\right)-z\right)^{-1}\right\|_{2} \leq N_{H S}(\gamma, v, R, z),
$$

where

$$
N_{H S}(\gamma, \nu, R, z)=\frac{C_{0} \pi}{\sqrt{6} \lambda_{1}} N_{A}(\gamma, \nu, R, z),
$$

where $N_{A}(\gamma, R, v, f, z)$ is as in (4.30).

Following Section 3 on page 44, we denote by

$$
\begin{gathered}
\lambda_{c}=-\frac{1}{2 \nu}\left[C_{1}(R+\zeta(R))+\gamma \nu \lambda_{1}^{1 / 2} C_{L}^{1 / 2}\right]^{2}, \\
\widetilde{M}=N_{7}\left(\gamma, 2 \lambda_{c}+\frac{2}{\left|\lambda_{c}\right|}, R, \nu, f\right),
\end{gathered}
$$

where $N_{7}$ is as (4.22).

Lemma 4.7. Let $R>0, u_{m} \in V$ with $\left\|u_{m}\right\| \leq R$, and $\rho, s>0$, and let $\Lambda \in \mathbb{C}$ satisfy $|\mathfrak{R} \Lambda| \leq s$. Then for every $m<\infty$, if

$$
\operatorname{dist}\left(\Lambda, \operatorname{Sp}\left(\mathcal{N}_{m}\left(u_{m}\right)\right)\right) \geq \rho,
$$


70 Chongsheng CaO, Ioannis G. Kevrekidis \& Edriss S. Titi then we have

$$
\begin{aligned}
\left|\left(\mathcal{N}_{m}\left(u_{m}\right)-\Lambda\right)^{-1} v\right| \leq N_{8}(\gamma, \rho, s, R, v, f)|v|, & \forall v \in H_{m}, \\
\left\|\left(\mathcal{N}_{m}\left(u_{m}\right)-\Lambda\right)^{-1} v\right\| \leq N_{9}(\gamma, \rho, s, R, v, f)|v|, & \forall v \in H_{m}, \\
\left|A\left(\mathcal{N}_{m}\left(u_{0}\right)-\Lambda\right)^{-1} v\right| \leq N_{10}(\gamma, \rho, s, R, v, f)|v|, & \forall v \in H_{m},
\end{aligned}
$$

where

$$
\begin{array}{ll}
N_{8}(\gamma, \rho, s, R, \nu, f) & \\
= \begin{cases}N_{H}(\gamma, R, \nu, f, z), & \Lambda \notin \sigma\left(R-\lambda_{c} / 2\right), \\
\exp \left\{\frac{1}{2}+\frac{5}{2}\left|s-2 \lambda_{c}\right|^{2} N_{H S}^{2}\left(\gamma, \nu, R,\left|s-2 \lambda_{c}\right|\right)\right\} & \\
\times \frac{1}{\lambda_{c}\left|\lambda-2 \lambda_{c}\right|^{2}}\left(\frac{2\left|s-\lambda_{c}\right|}{\rho}\right)^{\widetilde{M}}, & \Lambda \in \sigma\left(R-\lambda_{c} / 2\right),\end{cases}
\end{array}
$$

(4.38) $\quad N_{9}(\gamma, \rho, s, R, \nu, f)$

$$
\begin{aligned}
&=\left(\frac{2 N_{8}(\gamma, \rho, s, R, v, f)}{v}\right)^{1 / 2}+\left(\frac{2 s}{v}\right)^{1 / 2} N_{8}(\gamma, \rho, s, R, \nu, f) \\
&+\left[\frac{\sqrt{2} C_{1}\left(R+N_{4}(\gamma, R, \nu, f)\right)}{v}+\gamma \sqrt{3 C_{L} \lambda_{1}}+\frac{2 C_{1} \lambda_{1}^{1 / 4} R}{v \lambda_{m+1}^{1 / 4}}\right] \\
& \times N_{8}(\gamma, \rho, s, R, \nu, f),
\end{aligned}
$$

(4.39) $N_{10}(\gamma, \rho, s, R, \nu, f)$

$$
\begin{aligned}
&=\frac{1}{v}+\left(\frac{2 C_{1} R}{v}+\gamma C_{L}^{1 / 2} \lambda_{1}^{1 / 2}\right)^{2} N_{8}(\gamma, \rho, s, R, \nu, f) \\
&+\left[\left(\frac{2 s}{v}\right)^{1 / 2}+\frac{2 C_{1}}{v}\left(R+N_{4}(\gamma, R, \nu, f)\right)+\gamma C_{L}^{1 / 2} \lambda_{1}^{1 / 2}\right] \\
& \times N_{9}(\gamma, \rho, s, R, \nu, f),
\end{aligned}
$$

and $N_{H S}\left(\gamma, \nu, R,\left|s-2 \lambda_{c}\right|\right)$ is as in (4.31).

Proof. By using the same approach as for the estimate (3.26), we can get the estimate (4.34). We only need to show the estimates (4.35) and (4.36). 
Let $v_{m}=\Phi_{\text {app }}\left(u_{m}\right)$ and for each $g \in H_{m}$, let $\left(\mathcal{N}_{m}\left(u_{m}\right)-\lambda\right)^{-1} g=w$, i.e.,

$$
\begin{aligned}
v A w+P_{m}\left[B\left(u_{m}+v_{m}, w\right)+B\left(w, u_{m}+v_{m}\right)+B\left(u_{m}, \xi(w)\right)\right. \\
\left.+B\left(\xi(w), u_{m}\right)+\gamma L(w+\xi(w))\right]-\lambda w=g
\end{aligned}
$$

and

$$
v A \xi(w)+Q_{m}\left(B\left(u_{m}, w\right)+B\left(w, u_{m}\right)+\gamma L w\right)=0 .
$$

Taking the inner product, in $H$, of the equation (4.40) with $w$ and the equation (4.41) with $\xi(w)$, we obtain

$$
\begin{aligned}
& v\|w\|^{2}+\left(B\left(w, u_{m}+v_{m}\right), w\right)+\left(B\left(u_{m}, \xi(w)\right), w\right) \\
& \quad+\left(B\left(\xi(w), u_{m}\right), w\right)+\gamma(L(w+\xi(w)), w)-\lambda|w|^{2}=(g, w),
\end{aligned}
$$

and

$$
v\|\xi(w)\|^{2}+\left(B\left(u_{m}, w\right), \xi(w)\right)+\left(B\left(w, u_{m}\right), \xi(w)\right)+\gamma(L w, \xi(w))=0 .
$$

The summation of these two equations leads to

$$
\begin{aligned}
v\|w\|^{2}+v\|\xi\|^{2} \leq \mid & \left.(g, w)|+| \lambda|| w\right|^{2}+\left|\left(B\left(w, u_{m}+v_{m}\right), w\right)\right| \\
& +\left|\left(B\left(\xi(w), u_{m}\right), w\right)\right|+\left|\left(B\left(w, u_{m}\right), \xi(w)\right)\right| \\
& +\gamma|(L(w+\xi(w)), w)|+\gamma|(L w, \xi(w))| .
\end{aligned}
$$

By using (2.7)-(2.10) and (2.21), we reach

$$
\begin{gathered}
v\|w\|^{2}+v\|\xi\|^{2} \leq|g||w|+s|w|^{2}+\gamma v C_{L}^{1 / 2} \lambda_{1}^{1 / 2}|w|(\|w\|+\|\xi\|) \\
+C_{1}\left|u_{m}+v_{m}\right|^{1 / 2}\left|A\left(u_{m}+v_{m}\right)\right|^{1 / 2}|w|\|w\| \\
+\frac{2 C_{1} \lambda_{1}^{1 / 4} R}{\lambda_{m+1}^{1 / 4}}|w|\|\xi\| .
\end{gathered}
$$

By the Cauchy-Schwarz inequality, we conclude

$$
\begin{aligned}
\frac{v}{2}\|w\|^{2} \leq|g| & |w|+s|w|^{2}+\frac{3}{2} \gamma^{2} v C_{L} \lambda_{1}|w|^{2} \\
& +\frac{C_{1}^{2}\left|u_{m}+v_{m}\right|\left|A\left(u_{m}+v_{m}\right)\right||w|^{2}}{v}+\frac{2 C_{1}^{2} \lambda_{1}^{1 / 2} R^{2}}{\lambda_{m+1}^{1 / 2}}|w|^{2} .
\end{aligned}
$$


72 Chongsheng CaO, IoAnnis G. Kevrekidis \& Edriss S. Titi

By applying the estimate (4.34), we get (4.35).

Moreover, taking the inner product, in $H$, of the equation (4.40) with $A w$ gives

$$
\begin{aligned}
& v|A w|^{2} \leq s\|w\|^{2}+|(g, A w)| \\
&+\mid\left(B\left(u_{m}+v_{m}, w\right), A w\right)+\left(B\left(w, u_{m}+v_{m}\right), A w\right) \\
& \quad+\left(B\left(u_{m}, \xi\right), A w\right)+\left(B\left(\xi, u_{m}\right), A w\right)+\gamma(L w, A w) \mid .
\end{aligned}
$$

By using (2.7)-(2.10) and (2.20) with $\beta=\frac{1}{2}$, we get

$$
\begin{aligned}
v|A w|^{2} \leq s \| & w\left\|^{2}+|g||A w|+C_{1}\left|u_{m}+v_{m}\right|^{1 / 2}\left|A\left(u_{m}+v_{m}\right)\right|^{1 / 2}\right\| w \||A w| \\
& +C_{1}\left\|u_{m}+v_{m}\right\|^{1 / 2}\left|A\left(u_{m}+v_{m}\right)\right|^{1 / 2}|w|^{1 / 2}\|w\|^{1 / 2}|A w| \\
& +\frac{C_{1}\left|A u_{m}\right|\|\xi\|}{\lambda_{1}^{1 / 2}}|A w|+\frac{C_{1}\left|A u_{m}\right|\|\xi\|}{\lambda_{1}^{1 / 4} \lambda_{m+1}^{1 / 4}}|A w| \\
& +\gamma v C_{L}^{1 / 2} \lambda_{1}^{1 / 2}(\|w\|+\|\xi\|)|A w| .
\end{aligned}
$$

By using estimates (4.12) and (4.34), we obtain

$$
\|\xi(w)\| \leq\left(\frac{2 C_{1} R}{v}+\gamma C_{L}^{1 / 2} \lambda_{1}^{1 / 2}\right) N_{8}(\gamma, \rho, s, R, v, f)|g|,
$$

By the Cauchy-Schwarz inequality, and using estimates (4.34) and (4.35) and the above inequality, we get (4.36).

Lemma 4.8. Let $R>0$ and $u_{0}, u_{1} \in \mathcal{D}(A)$ with $\left|A u_{0}\right| \leq \lambda_{1}^{1 / 2} R,\left|A u_{1}\right| \leq$ $\lambda_{1}^{1 / 2} R$, and let $s, \rho>0$. Then there are two constants

$$
\eta(\gamma, \rho, s, R, \nu, f)=\frac{v}{2 C_{1} N_{8}(\gamma, \rho, s, R, \nu, f) N_{11}(\gamma, \rho, s, R, \nu, f)},
$$

and

$$
T_{2}(\gamma, \rho, s, R, v, f)=\frac{4 N_{8}^{2}(\gamma, \rho, s, R, v, f) N_{12}^{2}(\gamma, \rho, s, R, v, f)}{C_{0} v^{2}},
$$

where

(4.44) $\quad N_{11}(\gamma, \rho, s, R, \nu, f)=\frac{N_{5}^{1 / 2}(\gamma, s, R, \nu, f) N_{6}^{1 / 2}(\gamma, s, R, \nu, f)}{\lambda_{1}^{1 / 4}}$

$$
+v^{1 / 2} N_{6}^{1 / 2}(\gamma, s, R, v, f),
$$


(4.45) $N_{12}(\gamma, \rho, s, R, \nu, f)$

$$
\begin{aligned}
=C_{1} \lambda_{1}^{1 / 2}\{R+ & N_{4}(\gamma, R, \nu, f)\left[N_{5}(\gamma, s, R, \nu, f)+v^{1 / 2} N_{6}^{1 / 2}(\gamma, s, R, \nu, f)\right] \\
+4 C_{1} R & \left.N_{0}(\gamma, s, R, \nu, f)+2 C_{1} N_{6}(\gamma, s, R, \nu, f)\right\} \\
& +\gamma \nu C_{L}^{1 / 2} \lambda_{1}^{1 / 2}\left[N_{6}(\gamma, s, R, \nu, f)+2 N_{0}(\gamma, s, R, \nu, f)\right],
\end{aligned}
$$

and $N_{4}(\gamma, R, v, f), N_{5}(\gamma, s, R, \nu, f), N_{6}(\gamma, s, R, \nu, f)$, and $N_{8}(\gamma, \rho, s, R, \nu, f)$ are as in (4.11), (4.15), (4.16), and (4.37) respectively, and $N_{0}(\gamma, R, v, f)$ is as specified in (4.49), such that if

$$
\left\|P_{m}\left(u_{0}-u_{1}\right)\right\| \leq \eta(\gamma, \rho, s, R, v, f)
$$

and

$$
\left(\operatorname{Sp}\left(\mathcal{N}_{m}\left(P_{m} u_{0}\right)\right)\right)_{s}=\varnothing
$$

holds for some $m \in\left[T_{2}(\gamma, \rho, s, R, v, f), \infty\right)$, then for every $n \in[m, \infty]$ we have

$$
\left(\operatorname{Sp}\left(\mathcal{N}_{n}\left(P_{n} u_{1}\right)\right)\right)_{s} \subset B\left(\operatorname{Sp}\left(\mathcal{N}_{m}\left(P_{m} u_{0}\right)\right), \rho\right) .
$$

Proof. Let $u_{m}=P_{m} u_{0}, v_{m}=\Phi_{\text {app }}\left(u_{m}\right)$; and $p_{m}=P_{m} u_{1}, q_{m}=Q_{m} u_{1}$; and $\tilde{p}_{n}=P_{n} u_{1}, \widetilde{q}_{n}=\Phi_{\text {app }}\left(u_{n}\right)$. Suppose that $\lambda \in\left(\operatorname{Sp}\left(\mathcal{N}_{n}\left(\widetilde{p}_{n}\right)\right)\right)_{s}$, i.e., there is a $w \in H_{n},|w|=v$ such that $\mathcal{N}_{n}\left(\tilde{p}_{n}\right) w=\lambda w$ and $|\mathfrak{R} \lambda| \leq s$. Denote by

$$
w_{m}=P_{m} w \quad \text { and } \quad w_{m}^{\perp}=Q_{m} w
$$

If $\lambda \notin B\left(\operatorname{Sp}\left(\mathcal{N}_{m}\left(u_{m}\right)\right), \rho\right)$, i.e., $\operatorname{dist}\left(\lambda, \operatorname{Sp}\left(\mathcal{N}_{m}\left(u_{m}\right)\right)\right) \geq \rho$, by Lemma 4.7 on page 69 , and using the same argument as in Lemma 3.7 on page 53 , we get

$$
\begin{aligned}
\left|\mathcal{N}_{m}\left(u_{m}\right) w_{m}-\lambda w_{m}\right| & \geq \frac{\left|w_{m}\right|}{N_{8}(\gamma, \rho, s, \nu, R)} \\
& \geq \frac{1}{N_{8}(\gamma, \rho, s, \nu, R)}\left(\nu-\frac{N_{5}(\gamma, s, \nu, R)}{\lambda_{m+1}^{1 / 2}}\right) \\
& =: \mathcal{L} \mathcal{H},
\end{aligned}
$$

where $N_{5}(\gamma, s, R, v, f)$ is as in (4.15), and $N_{8}(\gamma, \rho, s, R, v, f)$ is as in (4.37). On the other hand, 
74 Chongsheng CaO, IoAnnis G. Kevrekidis \& Edriss S. Titi

$$
\begin{aligned}
\mathcal{N}_{m}\left(u_{m}\right) w_{m}-\lambda w_{m} \\
=\mathcal{N}_{m}\left(u_{m}\right) w_{m}-P_{m}\left(\mathcal{N}_{n}\left(\tilde{p}_{n}\right) w\right) \\
=P_{m}\left[B\left(u_{m}+v_{m}-\tilde{p}_{n}-\tilde{q}_{n}, w_{m}\right)+B\left(\tilde{p}_{n}+\tilde{q}_{n},-w_{m}^{\perp}\right)\right. \\
\quad+B\left(-w_{m}^{\perp}, u_{m}+v_{m}\right)+B\left(w, u_{m}+v_{m}-\tilde{p}_{n}-\tilde{q}_{n}\right)+B\left(u_{m}, \xi\left(w_{m}\right)\right) \\
\left.\quad+B\left(\xi\left(w_{m}\right), u_{m}\right)-B\left(\tilde{p}_{n}, \zeta(w)\right)-B\left(\zeta(w), \tilde{p}_{n}\right)-\gamma L w_{m}^{\perp}\right] \\
=P_{m}\left[B\left(u_{m}-p_{m}, w_{m}\right)+B\left(w, u_{m}-p_{m}\right)+B\left(\xi\left(w_{m}\right), u_{m}\right)+B\left(u_{m}, \xi\left(w_{m}\right)\right)\right. \\
\quad+B\left(v_{m}-q_{m}, w_{m}\right)+B\left(w, v_{m}-q_{m}\right)+B\left(\zeta(w), \tilde{p}_{n}\right)+B\left(\tilde{p}_{n}, \zeta(w)\right) \\
\left.\quad+B\left(-w_{m}^{\perp}, u_{m}+v_{m}\right)+B\left(\tilde{p}_{n}+\tilde{q}_{n},-w_{m}^{\perp}\right)-\gamma L w_{m}^{\perp}\right],
\end{aligned}
$$

where $\xi\left(w_{m}\right)$ and $\zeta(w)$ satisfy following equations

$$
\begin{aligned}
v A \xi+Q_{m}\left[B\left(u_{m}, w_{m}\right)+B\left(w_{m}, u_{m}\right)+\gamma L w_{m}\right] & =0, \\
v A \zeta+Q_{n}\left[B\left(\tilde{p}_{n}, w\right)+B\left(w, \tilde{p}_{n}\right)+\gamma L w\right] & =0 .
\end{aligned}
$$

By using (2.7), (2.8), and (2.20) with $\beta=\frac{1}{2}$, it is clear that

$$
\begin{aligned}
& |A \xi| \leq N_{0}(\gamma, s, R, \nu, f), \\
& |A \zeta| \leq N_{0}(\gamma, s, R, \nu, f),
\end{aligned}
$$

where

$$
\begin{aligned}
N_{0}(\gamma, s, R, \nu, f)=\frac{C_{1} R}{v}\left(N_{5}(\gamma, s, R, \nu, f)\right. & \left.+v^{1 / 2} \lambda_{1}^{1 / 2} N_{5}^{1 / 2}(\gamma, s, R, \nu, f)\right) \\
& +\gamma C_{L}^{1 / 2} \lambda_{1}^{1 / 2} N_{5}(\gamma, s, R, \nu, f) .
\end{aligned}
$$

By using the inequalities (2.8)-(2.10) and (2.20) with $\beta=\frac{1}{2}$, we obtain

$$
\begin{aligned}
\left|\left(\mathcal{N}_{m}\left(u_{m}\right)-\lambda\right) w_{m}\right| & \leq C_{1}\left\|u_{m}-p_{m}\right\|\left(\frac{\left\|w_{m}\right\|^{1 / 2}\left|A w_{m}\right|^{1 / 2}}{\lambda_{1}^{1 / 4}}+|w|^{1 / 2}|A w|^{1 / 2}\right) \\
& +C_{1}\left[\frac{\left|A v_{m}\right|+\left|A q_{m}\right|}{\lambda_{m+1}^{1 / 2}}\left(\left\|w_{m}\right\|+|w|^{1 / 2}|A w|^{1 / 2}\right)\right. \\
& \left.+\frac{2\left(|A \xi|\left|A u_{m}\right|+|A \zeta|\left|A \tilde{p}_{n}\right|\right)}{\lambda_{1}^{1 / 2} \lambda_{m+1}^{1 / 2}}\right] \\
& +\gamma \nu C_{L}^{1 / 2} \lambda_{1}^{1 / 2}\left[\left\|w_{m}^{\perp}\right\|+\|\xi\|+\|\zeta\|\right] \\
& +C_{1}\left[\frac{\left|A\left(\tilde{p}_{n}+\tilde{q}_{n}\right)\right|}{\lambda_{1}^{1 / 2}}\left\|w_{m}^{\perp}\right\|+\left|w_{m}^{\perp}\right|^{1 / 2}\left|A w_{m}^{\perp}\right|^{1 / 2}\left\|\tilde{p}_{n}+\tilde{q}_{n}\right\|\right] \leq
\end{aligned}
$$




$$
\begin{array}{r}
\leq C_{1}\left[\frac{N_{5}^{1 / 2}(\gamma, s, R, v, f) N_{6}^{1 / 2}(\gamma, s, R, \nu, f)}{\lambda_{1}^{1 / 4}}+v^{1 / 2} N_{6}^{1 / 2}(\gamma, s, R, v, f)\right]\left\|u_{m}-p_{m}\right\| \\
+\frac{1}{\lambda_{m+1}^{1 / 2}}\left[C _ { 1 } \lambda _ { 1 } ^ { 1 / 2 } \left(R+N_{4}(\gamma, R, v, f)\left(N_{5}(\gamma, s, R, v, f)+v^{1 / 2} N_{6}^{1 / 2}(\gamma, s, R, v, f)\right)\right.\right. \\
\left.+4 C_{1} R N_{0}(\gamma, s, R, v, f)+2 C_{1} N_{6}(\gamma, s, R, v, f)+\gamma v C_{L}^{1 / 2} \lambda_{1}^{1 / 2}\right) \\
\left.\times\left(N_{6}(\gamma, s, R, v, f)+2 N_{0}(\gamma, s, R, v, f)\right)\right]=: \mathcal{R H},
\end{array}
$$

where $N_{4}(\gamma, R, v, f), N_{5}(\gamma, s, R, v, f)$, and $N_{6}(\gamma, s, R, v, f)$ are as in (4.11), (4.15), and (4.16) respectively, and $N_{0}(\gamma, s, R, v, f)$ is as in (4.49). Therefore, we have

$$
\mathcal{L} \mathcal{H} \leq \mathcal{R} \mathcal{H}
$$

But if we let

$$
\left\|u_{m}-p_{m}\right\| \leq \eta(\gamma, \rho, s, R, \nu, f) \quad \text { and } \quad m \geq T_{2}(\gamma, \rho, s, R, \nu, f),
$$

where $\eta(\gamma, \rho, s, R, v, f)$ and $T_{2}(\gamma, s, \rho, R, v, f)$ are as in (4.42) and (4.43), then

$$
\begin{aligned}
& \mathcal{L} \mathcal{H}= \frac{1}{N_{8}(\gamma, \rho, s, R, v, f)}\left[\nu-\frac{N_{5}(\gamma, s, v, R)}{\lambda_{m+1}^{1 / 2}}\right] \geq \mathcal{R} \mathcal{H} \\
&=C_{1}\left[\frac{N_{5}^{1 / 2}(\gamma, s, R, \nu, f) N_{6}^{1 / 2}(\gamma, s, R, v, f)}{\lambda_{1}^{1 / 4}}+v^{1 / 2} N_{6}^{1 / 2}(\gamma, s, R, \nu, f)\right]\left\|u_{m}-p_{m}\right\| \\
& \quad+\frac{1}{\lambda_{m+1}^{1 / 2}}\left[C _ { 1 } \lambda _ { 1 } ^ { 1 / 2 } \left(R+N_{4}(\gamma, R, v, f)\left(N_{5}(\gamma, s, R, v, f)+v^{1 / 2} N_{6}^{1 / 2}(\gamma, s, R, v, f)\right)\right.\right. \\
&\left.+4 C_{1} R N_{0}(\gamma, s, R, v, f)+2 C_{1} N_{6}(\gamma, s, R, v, f)+\gamma v C_{L}^{1 / 2} \lambda_{1}^{1 / 2}\right) \\
&\left.\quad \times\left(N_{6}(\gamma, s, R, v, f)+2 N_{0}(\gamma, s, R, v, f)\right)\right] .
\end{aligned}
$$

This leads into contradiction. Thus $\lambda \in B\left(\operatorname{Sp}\left(\mathcal{N}_{m}\left(u_{0}\right)\right), \rho\right)$, i.e.,

$$
\left(\operatorname{Sp}\left(\mathcal{N}_{n}\left(u_{1}\right)\right)\right)_{s} \subset B\left(\operatorname{Sp}\left(\mathcal{N}_{m}\left(u_{0}\right)\right), \rho\right),
$$

which completes the proof of the lemma. 
76 Chongsheng CaO, IoAnnis G. Kevrekidis \& Edriss S. Titi

4.2. The main result in the nonlinear Galerkin case. Next we present our main theorem of this section.

Theorem 4.1. Let $\sigma>0$ be given, and let $p_{m}^{\infty}+q_{m}^{\infty}$ be a steady state solution of the Galerkin approximation equation (4.1) of the closed-loop system (2.18) such that $\left|p_{m}^{\infty}+q_{m}^{\infty}\right| \leq N_{1}$. Then there exists a constant

(4.50) $M_{\mathrm{NLG}}\left(\gamma, \sigma, N_{1}, \nu, f\right)=\max \left\{T_{3}\left(\gamma, \sigma, N_{1}, \nu, f\right), T_{4}\left(\gamma, \sigma, N_{1}, \nu, f\right)\right\}$,

where $T_{3}\left(\gamma, \sigma, N_{1}, \nu, f\right), T_{4}\left(\gamma, \sigma, N_{1}, \nu, f\right)$ are specified as in (4.70) and (4.74), such that if

$$
\left(\operatorname{Sp}\left(\mathcal{N}_{m}\left(p_{m}^{\infty}\right)\right)\right)_{\sigma}=\varnothing
$$

holds for some $m \geq M_{\mathrm{NLG}}\left(\gamma, \sigma, N_{1}, v, f\right)$, then we have

(i) For every $n \geq m$, there is a stationary solution $p_{n}^{\infty}+q_{n}^{\infty}$ of the nonlinear Galerkin approximation equations (4.1) and (4.2) of order $n$, for which

$$
\begin{aligned}
& \left(\operatorname{Sp}\left(\mathcal{N}_{n}\left(p_{n}^{\infty}\right)\right)\right)_{\sigma / 2}=\varnothing, \\
& \left|p_{n}^{\infty}+q_{n}^{\infty}-p_{m}^{\infty}-q_{m}^{\infty}\right| \leq \frac{N_{13}(\gamma, \sigma, v, f)}{\lambda_{m+1}^{3 / 2}},
\end{aligned}
$$

where

$$
\begin{aligned}
& N_{13}\left(\gamma, \sigma, N_{1}, \nu, f\right) \\
& =\frac{4}{3}\left[\frac{\lambda_{1} N_{4}\left(\gamma, N_{3}, v, f\right)}{v}\right. \\
& \times\left(2 C_{1} N_{3}+\gamma v C_{L}^{1 / 2} \lambda_{1}^{1 / 2}\right)\left(\gamma \nu C_{L}^{1 / 2}+\frac{1}{\lambda_{1}^{1 / 2}}+\frac{2 C_{1} N_{4}^{2}\left(\gamma, N_{3}, \nu, f\right)}{\lambda_{m+1}^{1 / 2}}\right) \\
& +2 C_{1} \lambda_{1} N_{4}^{2}\left(\gamma, N_{3}, \nu, f\right) \\
& +\frac{\lambda_{1}\left(\left(1+\lambda_{1}^{1 / 2}\right) \lambda_{m+1}^{1 / 2}+2 C_{1} \lambda_{1}^{1 / 2} N_{4}^{2}\left(\gamma, N_{3}, \nu, f\right) N_{10}\left(\gamma, \sigma / 2, \sigma, N_{3}, \nu, f\right)\right)}{2 C_{1} \lambda_{m+1}^{1 / 2} v N_{10}^{2}\left(\gamma, \sigma / 2, \sigma, N_{3}, \nu, f\right)} \\
& \times\left(4 C_{1} N_{3}+\gamma \nu C_{L}^{1 / 2} \lambda_{1}^{1 / 2}\right) \\
& +\frac{\lambda_{1}^{3 / 2}}{32 C_{1}^{2} \nu N_{10}^{2}\left(\gamma, \sigma / 2, \sigma, N_{3}, \nu, f\right)}\left(\lambda_{1}^{1 / 2}+2 N_{10}\left(\gamma, \frac{\sigma}{2}, \sigma, N_{3}, \nu, f\right)\right. \\
& \left.\left.\times\left(2 C_{1} N_{3}+\gamma v C_{L}^{1 / 2} \lambda_{1}^{1 / 2}+1+\frac{2 C_{1} \lambda_{1}^{1 / 2} N_{4}^{2}\left(\gamma, N_{3}, v, f\right)}{\lambda_{m+1}^{1 / 2}}\right)\right)\right] \\
& \times N_{9}\left(\gamma, \frac{\sigma}{2}, \sigma, N_{3}, \nu, f\right),
\end{aligned}
$$


where $N_{9}\left(\gamma, \sigma / 2, \sigma, N_{3}, \nu, f\right)$ and $N_{10}\left(\gamma, \sigma / 2, \sigma, N_{3}, v, f\right)$ are as (4.39) and (4.39) respectively.

(ii) There is a stationary solution $u^{\infty}$ of the closed-loop system (2.18) such that

$$
p_{n}^{\infty}+q_{n}^{\infty} \rightarrow u^{\infty} \text { as } n \rightarrow \infty .
$$

Moreover, $u^{\infty}$ is hyperbolic and

$$
\begin{gathered}
\left(\operatorname{Sp}\left(\mathcal{N}_{\infty}\left(u^{\infty}\right)\right)\right)_{\sigma / 2}=\varnothing, \\
\left|u^{\infty}-p_{n}^{\infty}-q_{n}^{\infty}\right| \leq \frac{N_{13}(\gamma, \sigma, v, f)}{\lambda_{n+1}^{3 / 2}} .
\end{gathered}
$$

Proof. We will use the contraction mapping theorem to show the existence of $p_{n}^{\infty}+q_{n}^{\infty}$.

First let us establish the map. Denote by

$$
\begin{aligned}
w=p_{n}^{\infty}+q_{n}^{\infty}-p_{m}^{\infty}-q_{m}^{\infty}, \quad w_{n}=P_{n} w, \quad w_{n}^{\perp}=Q_{n} w, \quad \hat{q}_{m}^{\infty}=P_{n} q_{m}^{\infty}, \\
\tilde{q}_{m}^{\infty}=Q_{n} q_{m}^{\infty}, \quad u_{n}=p_{m}^{\infty}+\hat{q}_{m}^{\infty}, \quad v_{n}=\Phi_{\text {app }}\left(u_{n}\right) .
\end{aligned}
$$

If $p_{n}^{\infty}+q_{n}^{\infty}$ is a stationary solution of the nonlinear Galerkin approximation equations (4.1) and (4.2) based on $n$-eigenmodes, then $w$ should satisfy

$$
\begin{gathered}
v A\left(u_{n}+w_{n}\right)+P_{n}\left[B\left(p_{m}^{\infty}+q_{m}^{\infty}+w, p_{m}^{\infty}+q_{m}^{\infty}+w\right)-B\left(\tilde{q}_{m}^{\infty}+w_{n}^{\perp}, \tilde{a}_{m}^{\infty}+w_{n}^{\perp}\right)\right. \\
\left.+\gamma P_{n} L\left(u_{n}+v_{n}+w_{n}+w_{n}^{\perp}\right)\right]=P_{n} f, \\
v A\left(\tilde{q}_{m}^{\infty}+w_{n}^{\perp}\right)+Q_{n}\left[B\left(u_{n}+w_{n}, u_{n}+w_{n}\right)+\gamma L\left(u_{n}+w_{n}\right)\right]=Q_{n} f,
\end{gathered}
$$

that is,

$$
\begin{aligned}
& \mathcal{N}_{n}\left(u_{n}\right) w_{n}=v A w_{n}+P_{n}\left[B\left(u_{n}+v_{n}, w_{n}\right)+B\left(w_{n}, u_{n}+v_{n}\right)\right. \\
& \left.\quad+B\left(u_{n}, \xi\left(w_{n}\right)\right)+B\left(\xi\left(w_{n}\right), u_{n}\right)+\gamma L\left(w_{n}+\xi\left(w_{n}\right)\right)\right]=\Psi\left(w_{n}\right),
\end{aligned}
$$

where 


$$
\begin{aligned}
\Psi\left(w_{n}\right) \\
=P_{n}\left[B\left({q_{m}^{\infty}}^{\infty}, q_{m}^{\infty}\right)-B\left(\tilde{q}_{m}^{\infty}, \tilde{q}_{m}^{\infty}\right)+B\left(\hat{q}_{m}^{\infty}, w_{n}^{\perp}\right)\right. \\
+B\left(w_{n}^{\perp}, \hat{q}_{m}^{\infty}\right)+B\left(u_{n}, \xi\left(w_{n}\right)+v_{n}-\tilde{q}_{m}^{\infty}-w_{n}^{\perp}\right) \\
+B\left(\xi\left(w_{n}\right)+v_{n}-\tilde{q}_{m}^{\infty}-w_{n}^{\perp}, u_{n}\right)+B\left(w_{n}, v_{n}-\tilde{q}_{m}^{\infty}-w_{n}^{\perp}\right) \\
\left.+B\left(v_{n}-\tilde{q}_{m}^{\infty}-w_{n}^{\perp}, w_{n}\right)-B\left(w_{n}, w_{n}\right)+\gamma L\left(\xi\left(w_{n}\right)-w_{n}^{\perp}\right)\right] .
\end{aligned}
$$

From (4.61), we get

$$
w_{n}^{\perp}=v_{n}-\tilde{q}_{m}^{\infty}+\xi\left(w_{n}\right)-(\nu A)^{-1} Q_{n} B\left(w_{n}, w_{n}\right) .
$$

In other words, if we can show the existence of $w_{n}$, then we get $w_{n}^{\perp}$ by (4.63). By Lemma 4.8 on page 72 , we have $\left(\operatorname{Sp}\left(\mathcal{N}_{n}\left(u_{n}\right)\right)\right)_{\sigma / 2}=\varnothing$, when $m \geq$ $T_{2}\left(\sigma, \sigma / 2, v, N_{3}\right)$. So $\mathcal{N}_{n}\left(u_{n}\right)$ is invertible. Denote by

$$
\mathcal{F}\left(w_{n}\right)=\left(\mathcal{N}_{n}\left(u_{n}\right)\right)^{-1} \Psi\left(w_{n}\right),
$$

where $\Psi\left(w_{n}\right)$ is as in (4.62).

Next we will show that $\mathcal{F}$ maps

$$
\mathcal{B}_{n}(r)=\left\{w_{n} \in H_{n}:\left|A w_{n}\right| \leq r\right\}
$$

into $\mathcal{B}_{n}(r)$, where

$$
r=\frac{\lambda_{1}}{4 C_{1} N_{10}\left(\gamma, \sigma / 2, \sigma, N_{3}, \nu, f\right)} .
$$

By the estimate (3.28) of the Lemma 4.7 on page 69, we get

$$
\left|A \mathcal{F}\left(w_{n}\right)\right| \leq N_{10}\left(\gamma, \frac{\sigma}{2}, \sigma, N_{3}, \nu, f\right)\left|\Psi\left(w_{n}\right)\right| .
$$

Now we will get an estimate for $\left|\Psi\left(w_{n}\right)\right|$. First, by using (2.7)-(2.11) and (2.20) with $\beta=\frac{1}{2}$, we get the following estimates

$$
\begin{aligned}
& \left|A \xi\left(w_{n}\right)\right| \leq \frac{4 C_{1} N_{3}+\gamma \nu C_{L}^{1 / 2} \lambda_{1}^{1 / 2}}{\nu \lambda_{m+1}^{1 / 2}}\left|A w_{n}\right| \\
& \left|A\left(v_{n}-\tilde{q}_{m}^{\infty}\right)\right| \leq \frac{\lambda_{1}^{1 / 2} N_{4}\left(\gamma, N_{3}, \nu, f\right)\left(2 C_{1} N_{3}+\gamma v C_{L}^{1 / 2} \lambda_{1}^{1 / 2}\right)}{\nu \lambda_{m+1}^{1 / 2}} \\
& \left|A w_{n}^{\perp}\right| \leq \frac{1}{v \lambda_{m+1}^{1 / 2}}\left(\lambda_{1}^{1 / 2} N_{4}\left(\gamma, N_{3}, \nu, f\right)\left(2 C_{1} N_{3}+\gamma \nu C_{L}^{1 / 2} \lambda_{1}^{1 / 2}\right)\right. \\
& \left.+\left(4 C_{1} N_{3}+\gamma v C_{L}^{1 / 2} \lambda_{1}^{1 / 2}\right)\left|A w_{1}\right|+\frac{2 C_{1}\left|A w_{1}\right|^{2}}{\lambda_{1}^{1 / 2}}\right) .
\end{aligned}
$$


Moreover, we use (2.7)-(2.11), (2.20) with $\beta=\frac{1}{2}$, and the above estimates to obtain

$$
\begin{aligned}
& \left|\Psi\left(w_{n}\right)\right| \\
& \leq \frac{2 C_{1} N_{3}\left|A\left(\xi\left(w_{n}\right)+v_{n}-\tilde{q}_{m}^{\infty}-w_{2}\right)\right|}{\lambda_{m+1}^{1 / 2}}+\frac{2 C_{1} \lambda_{1} N_{4}^{2}(\gamma, v, f)}{\lambda_{m+1}} \\
& +\frac{2 C_{1} \lambda_{1}^{1 / 2} N_{4}\left(\gamma, N_{3}, \nu, f\right)\left|A w_{n}^{\perp}\right|}{\lambda_{m+1}}+\frac{2 C_{1}\left|A w_{n}\right|}{\lambda_{1}^{1 / 2} \lambda_{m+1}^{1 / 2}}\left|A\left(v_{n}-\widetilde{q}_{m}^{\infty}-w_{n}^{\perp}\right)\right| \\
& +\frac{C_{1}\left|A w_{n}\right|^{2}}{\lambda_{1}}+\gamma \nu C_{L}^{1 / 2} \lambda_{1}^{1 / 2}\left\|\xi\left(w_{n}\right)-w_{n}^{\perp}\right\| \\
& \leq \frac{4 C_{1}^{2}}{v \lambda_{1} \lambda_{m+1}}\left|A w_{n}\right|^{3}+\frac{2 C_{1} \lambda_{1}^{1 / 2} N_{4}\left(\gamma, N_{3}, v, f\right)}{v \lambda_{m+1}^{3 / 2}}\left(4 C_{1} N_{3}+\gamma v C_{L}^{1 / 2} \lambda_{1}^{1 / 2}\right)\left|A w_{n}\right| \\
& +\frac{C_{1}\left|A w_{n}\right|^{2}}{\lambda_{1}}\left[1+\frac{1}{\lambda_{m+1}}\left(\frac{12 C_{1} \lambda_{1}^{1 / 2} N_{3}}{v}+2 \gamma C_{L}^{1 / 2} \lambda_{1}\right.\right. \\
& \left.\left.+\frac{4 C_{1} \lambda_{1} N_{4}\left(\gamma, N_{3}, v, f\right)}{v \lambda_{m+1}^{1 / 2}}\right)\right] \\
& +\frac{\lambda_{1} N_{4}\left(\gamma, N_{3}, v, f\right)}{\lambda_{m+1}}\left(2 C_{1} N_{3}+\gamma \nu C_{L}^{1 / 2} \lambda_{1}^{1 / 2}\right) \\
& \times\left(\frac{2 C_{1} N_{4}\left(\gamma, N_{3}, v, f\right)}{v \lambda_{m+1}^{1 / 2}}+\gamma C_{L}^{1 / 2}\right)+\frac{2 C_{1} \lambda_{1} N_{4}^{2}\left(\gamma, N_{3}, v, f\right)}{\lambda_{m+1}} .
\end{aligned}
$$

Therefore when $\left|A w_{n}\right| \leq r, r$ is as in (4.66), and

$$
m \geq T_{3}\left(\gamma, \sigma, N_{1}, \nu, f\right)=\max \left\{T_{2}\left(\sigma, \frac{\sigma}{2}, \nu, N_{3}\right), T_{5}, T_{6}\right\},
$$

where

$$
\begin{aligned}
& T_{5}=\left[\frac{16 C_{1} N_{10}^{2}\left(\gamma, \sigma / 2, \sigma, N_{3}, \nu, f\right) N_{14}\left(\gamma, \sigma, N_{1}, \nu, f\right)}{3 C_{0} \lambda_{1}}\right], \\
& T_{6}=\left[\frac{3 N_{15}\left(\gamma, \sigma, N_{1}, \nu, f\right)}{C_{0}}\right],
\end{aligned}
$$

and 
(4.71) $N_{14}\left(\gamma, \sigma, N_{1}, v, f\right)$

$$
\begin{gathered}
=\frac{\lambda_{1}}{16 C_{1} v N_{10}^{3}\left(\gamma, \sigma / 2, \sigma, N_{3}, v, f\right)}+2 C_{1} N_{4}^{2}\left(\gamma, N_{3}, \nu, f\right) \\
+\frac{12 C_{1} \lambda_{1}^{1 / 2} N_{3}+2 \gamma v C_{L}^{1 / 2} \lambda_{1}+4 C_{1} \lambda_{1}^{1 / 2} N_{4}\left(\gamma, N_{3}, v, f\right)}{16 C_{1} v N_{10}^{2}\left(\gamma, \sigma / 2, \sigma, N_{3}, \nu, f\right)} \\
+N_{4}\left(\gamma, N_{3}, \nu, f\right)\left(2 C_{1} N_{3}+\gamma v C_{L}^{1 / 2} \lambda_{1}^{1 / 2}\right) \\
\times\left(\frac{2 C_{1} N_{4}\left(\gamma, N_{3}, \nu, f\right)}{v \lambda_{1}^{1 / 2}}+\gamma C_{L}^{1 / 2}\right) \\
+\frac{N_{4}\left(\gamma, N_{3}, v, f\right)\left(4 C_{1} N_{3}+\gamma v C_{L}^{1 / 2} \lambda_{1}^{1 / 2}\right)}{2 v N_{10}\left(\gamma, \sigma / 2, \sigma, N_{3}, v, f\right)},
\end{gathered}
$$

(4.72) $\quad N_{15}\left(\gamma, \sigma, N_{1}, \nu, f\right)$

$$
\begin{aligned}
=2 \gamma & C_{1} C_{L}^{1 / 2}+\frac{3 C_{1}}{4 v N_{10}\left(\gamma, \sigma / 2, \sigma, N_{3}, \nu, f\right)} \\
& +\frac{2 C_{1}^{2}}{v \lambda_{1}^{1 / 2}\left(3 N_{3}+N_{4}\left(\gamma, N_{3}, \nu, f\right)\right)} \\
+\frac{2 C_{1}^{2} N_{4}\left(\gamma, N_{3}, \nu, f\right) N_{10}\left(\gamma, \sigma / 2, \sigma, N_{3}, \nu, f\right)}{\nu \lambda_{1}} & \times\left(4 C_{1} N_{3}+\gamma \nu C_{L}^{1 / 2} \lambda_{1}^{1 / 2}\right),
\end{aligned}
$$

we have $\left|A \mathcal{F}\left(w_{1}\right)\right| \leq r$, that is, $\mathcal{F}$ maps $\mathcal{B}_{n}(r)$ into $\mathcal{B}_{n}(r)$.

Finally, we will show that the map $\mathcal{F}$ is a contraction map on $\mathcal{B}_{n}(r)$. Suppose $w_{n}, \widetilde{w}_{n} \in \mathcal{B}_{n}(r)$. Then

$$
\begin{array}{rl}
\mathcal{F}\left(w_{n}\right)-\mathcal{F}\left(\widetilde{w}_{n}\right) & \\
=\left(\mathcal{N}_{n}\left(u_{n}\right)\right)^{-1} \Psi( & \left.w_{n}\right)-\left(\mathcal{N}_{n}\left(u_{n}\right)\right)^{-1} \Psi\left(\widetilde{w}_{n}\right) \\
=\left(\mathcal{N}_{n}\left(u_{n}\right)\right)^{-1} P_{n} & B\left(u_{n}, \xi\left(w_{n}\right)-\xi\left(\widetilde{w}_{1}\right)-w_{n}^{\perp}+\widetilde{w}_{n}^{\perp}\right)+B\left(\hat{q}_{m}^{\infty}, w_{n}^{\perp}-\widetilde{w}_{n}^{\perp}\right) \\
& +B\left(\xi\left(w_{n}\right)-\xi \widetilde{w}_{n}-w_{n}^{\perp}+\widetilde{w}_{n}^{\perp}, u_{n}\right) \\
& +B\left(w_{n}^{\perp}-\widetilde{w}_{n}^{\perp}, \hat{q}_{m}^{\infty}\right)-B\left(\widetilde{w}_{n}, w_{n}^{\perp}-\widetilde{w}_{n}^{\perp}\right) \\
& -B\left(w_{n}^{\perp}-\widetilde{w}_{n}^{\perp}, \widetilde{w}_{n}\right)+B\left(w_{n}-\widetilde{w}_{n}, v_{n}-\widetilde{q}_{m}^{\infty}-w_{n}^{\perp}\right) \\
& +B\left(v_{n}-\widetilde{q}_{m}^{\infty}-w_{n}^{\perp}, w_{n}-\widetilde{w}_{n}\right)-B\left(w_{n}-\widetilde{w}_{n}, w_{n}\right) \\
& \left.-B\left(\widetilde{w}_{n}, w_{n}-\widetilde{w}_{n}\right)+\gamma L\left(\xi\left(w_{n}\right)-\xi\left(\widetilde{w}_{n}\right)-w_{n}^{\perp}+\widetilde{w}_{n}^{\perp}\right)\right],
\end{array}
$$




$$
\begin{aligned}
& v A\left(\xi\left(w_{n}\right)-\xi\left(\widetilde{w}_{n}\right)\right) \\
& \quad+Q_{n}\left[B\left(u_{n}, w_{n}-\widetilde{w}_{n}\right)+B\left(w_{n}-\widetilde{w}_{n}, u_{n}\right)+\gamma L\left(w_{n}-\widetilde{w}_{n}\right)\right]=0, \\
& v A\left(w_{n}^{\perp}-\widetilde{w}_{n}^{\perp}\right) \\
& \quad=v A\left(\xi\left(w_{n}\right)-\xi\left(\widetilde{w}_{n}\right)\right)-Q_{n}\left[B\left(w_{n}-\widetilde{w}_{n}, w_{n}\right)+B\left(\widetilde{w}_{n}, w_{n}-\widetilde{w}_{n}\right)\right] .
\end{aligned}
$$

Then by using (2.7)-(2.11) and (2.20) with $\beta=\frac{1}{2}$, we obtain

$$
\begin{aligned}
\left|A\left(\xi\left(w_{n}\right)-\xi\left(\widetilde{w}_{n}\right)\right)\right| & \leq \frac{4 C_{1} N_{3}+\gamma v C_{L}^{1 / 2} \lambda_{1}^{1 / 2}}{v \lambda_{m+1}^{1 / 2}}\left|A\left(w_{n}-\widetilde{w}_{n}\right)\right|, \\
\left|A\left(w_{n}^{\perp}-\widetilde{w}_{n}^{\perp}\right)\right| & \leq \frac{4 C_{1} \lambda_{1}^{1 / 2} N_{3}+\gamma v C_{L}^{1 / 2} \lambda_{1}+4 C_{1}\left|A w_{n}\right|}{v \lambda_{1}^{1 / 2} \lambda_{m+1}^{1 / 2}}\left|A\left(w_{n}-\widetilde{w}_{n}\right)\right| .
\end{aligned}
$$

As a result of the estimates above, (2.7)-(2.11), and (2.20) with $\beta=\frac{1}{2}$, we have

$$
\begin{aligned}
& \left|A\left(\mathcal{F}\left(w_{n}\right)-\mathcal{F}\left(\widetilde{w}_{n}\right)\right)\right| \\
& \leq C_{1} N_{10}\left(\gamma, \frac{\sigma}{2}, \sigma, N_{3}, \nu, f\right)\left[\frac{2\left|A w_{n}\right|}{\lambda_{1}}+\frac{12 C_{1}^{2}\left|A w_{1}\right|^{2}}{v \lambda_{1} \lambda_{m+1}}+\frac{8 C_{1} \gamma C_{L}^{1 / 2}\left|A w_{n}\right|}{\lambda_{m+1}}\right. \\
& +\frac{8 C_{1}^{2}\left|A w_{n}\right|}{v \lambda_{m+1}}\left(\frac{3 N_{3}}{\lambda_{1}^{1 / 2}}+\frac{N_{4}\left(\gamma, \gamma, N_{3}, v, f\right)}{\lambda_{m+1}^{1 / 2}}\right) \\
& \left.+\frac{2 C_{1} \lambda_{1}^{1 / 2} N_{4}\left(\gamma, N_{3}, v, f\right)}{v \lambda_{m+1}^{3 / 2}}\left(4 C_{1} N_{3}+\gamma v C_{L}^{1 / 2} \lambda_{1}^{1 / 2}\right)\right] .
\end{aligned}
$$

Therefore, by (4.66) and (4.70), we get

$$
\left|A\left(\mathcal{F}\left(w_{1}\right)-\mathcal{F}\left(\widetilde{w}_{1}\right)\right)\right| \leq \frac{5}{6}\left|A\left(w_{1}-\widetilde{w}_{1}\right)\right| .
$$

Thus, $\mathcal{F}$ is a contraction map on $\mathcal{B}_{n}(r)$. By the contraction mapping theorem, there are a unique $w_{n}^{*} \in \mathcal{B}_{n}(r)$ and a unique $w_{n}^{* \perp}$ given by (4.63), such that

$$
p_{n}^{\infty}+q_{n}^{\infty}=p_{m}^{\infty}+q_{m}^{\infty}+w_{n}+w_{n}^{* \perp}
$$

is a steady state of the nonlinear Galerkin equations (4.1) and (4.2) of order $n$, and

$$
\left|A\left(p_{n}^{\infty}+q_{n}^{\infty}-p_{m}^{\infty}-q_{m}^{\infty}\right)\right| \leq \frac{\lambda_{1}}{4 C_{1} N_{10}\left(\gamma, \sigma / 2, \sigma, N_{3}, v, f\right)},
$$


when $m \geq T_{3}\left(\gamma, \sigma, N_{1}, v, f\right)$ and $T_{3}\left(\gamma, \sigma, N_{1}, \nu, f\right)$ is as in (4.70).

Now we will show the estimate (4.53). First notice that, by using the same argument as in the proof of (3.56) and (3.57), for all $w_{1}, w_{2} \in H_{m}$, we obtain

$$
\begin{aligned}
& \left|\left(\mathcal{N}_{m}\left(p_{m}^{\infty}\right)\right)^{-1} B\left(w_{1}, w_{2}\right)\right| \leq C_{1} N_{9}\left(\gamma, \frac{\sigma}{2}, \sigma, N_{3}, v, f\right)\left|w_{1}\right|^{1 / 2}\left|A w_{1}\right|^{1 / 2}\left|w_{2}\right| \\
& \left|\left(\mathcal{N}_{m}\left(p_{m}^{\infty}\right)\right)^{-1} B\left(w_{1}, w_{2}\right)\right| \leq C_{1} N_{9}\left(\gamma, \frac{\sigma}{2}, \sigma, N_{3}, \nu, f\right)\left|w_{1}\right|\left|w_{2}\right|^{1 / 2}\left|A w_{2}\right|^{1 / 2}
\end{aligned}
$$

Then we apply the above estimates and the estimate (4.36) of the Lemma (4.7) to (4.64), and obtain

$$
\begin{aligned}
\left|w_{n}^{*}\right|=\mid & \mathcal{F}\left(w_{n}^{*}\right) \mid \\
\leq & C_{1} N_{9}\left(\gamma, \frac{\sigma}{2}, \sigma, N_{3}, v, f\right)\left[2 N_{3}\left|\xi\left(w_{n}^{*}\right)+v_{n}-\tilde{q}_{m}^{\infty}-w_{n}^{* \perp}\right|\right. \\
& +\frac{2 \lambda_{1} N_{4}^{2}\left(\gamma, N_{3}, v, f\right)}{\lambda_{m+1}^{3 / 2}}+\frac{2 \lambda_{1}^{1 / 2} N_{4}^{2}\left(\gamma, N_{3}, v, f\right)\left|w_{2}\right|}{\lambda_{m+1}^{1 / 2}} \\
& \left.+\frac{2 r\left(\left|v_{n}-\tilde{q}_{m}^{\infty}-w_{n}^{* \perp}\right|\right)}{\lambda_{1}^{1 / 2}}\right] \\
& +\frac{C_{1} N_{10}\left(\gamma, \sigma / 2, \sigma, N_{3}, v, f\right)\left|w_{n}^{*}\right|^{3 / 2}\left\|w_{n}^{*}\right\|^{1 / 2}}{\lambda_{1}^{1 / 4}} \\
& +\gamma \nu C_{L}^{1 / 2} \lambda_{1}^{1 / 2} N_{9}\left(\gamma, \frac{\sigma}{2}, \sigma, N_{3}, \nu, f\right)\left|\xi\left(w_{1}\right)-w_{2}\right| .
\end{aligned}
$$

Since $\left|w_{n}^{*}\right|^{1 / 2}\left\|w_{n}^{*}\right\|^{1 / 2} \leq\left|A w_{n}^{*}\right| / \lambda_{1}^{3 / 4}$ and $\left(C_{1} r N_{10}\left(\gamma, \sigma / 2, \sigma, N_{3}, v, f\right)\right) / \lambda_{1}=$ $\frac{1}{4}$, we get

$$
\begin{aligned}
\left|w_{n}^{*}\right| \leq \frac{4}{3} N_{9} & \left(\gamma, \frac{\sigma}{2}, \sigma, N_{3}, v, f\right)\left[2 C_{1} N_{3}\left|(\nu A)^{-1} Q_{n} B\left(w_{n}^{*}, w_{n}^{*}\right)\right|\right. \\
& +\frac{2 C_{1} \lambda_{1}^{1 / 2} N_{4}^{2}\left(\gamma, N_{3}, v, f\right)\left|w_{n}^{* \perp}\right|}{\lambda_{m+1}^{1 / 2}} \\
& +\frac{2 r C_{1}\left(\left|\xi\left(w_{n}^{*}\right)\right|+\left|(v A)^{-1} Q_{n} B\left(w_{n}^{*}, w_{n}^{*}\right)\right|\right)}{\lambda_{1}^{1 / 2}} \\
& +\frac{2 C_{1} \lambda_{1} N_{4}^{2}\left(\gamma, N_{3}, v, f\right)}{\lambda_{m+1}^{3 / 2}} \\
& \left.+\gamma v C_{L}^{1 / 2} \lambda_{1}^{1 / 2}\left(\left|v_{n}-\tilde{q}_{m}^{\infty}\right|+\left|(v A)^{-1} Q_{n} B\left(w_{n}^{*}, w_{n}^{*}\right)\right|\right)\right] .
\end{aligned}
$$


Therefore, by using the estimates (4.67), (4.68), (4.69), and (4.73), we obtain

$$
\left|w_{n}^{*}\right| \leq \frac{N_{16}\left(\gamma, \sigma, N_{1}, v, f\right)}{\lambda_{m+1}^{3 / 2}},
$$

where

$$
\begin{aligned}
& N_{16}\left(\gamma, \sigma, N_{1}, \nu, f\right)=\frac{4}{3} N_{9}\left(\gamma, \frac{\sigma}{2}, \sigma, N_{3}, \nu, f\right) \\
& \times\left[\frac{\lambda_{1} N_{4}\left(\gamma, N_{3}, \nu, f\right)\left(2 C_{1} N_{3}+\gamma \nu C_{L}^{1 / 2} \lambda_{1}^{1 / 2}\right)}{v}\left(\gamma \nu C_{L}^{1 / 2}+\frac{2 C_{1} N_{4}^{2}\left(\gamma, N_{3}, \nu, f\right)}{\lambda_{m+1}^{1 / 2}}\right)\right. \\
& +2 C_{1} \lambda_{1} N_{4}^{2}\left(\gamma, N_{3}, \nu, f\right) \\
& +\frac{\lambda_{1}^{3 / 2}\left(\lambda_{m+1}^{1 / 2}+2 C_{1} N_{4}^{2}\left(\gamma, N_{3}, v, f\right) N_{10}\left(\gamma, \sigma / 2, \sigma, N_{3}, v, f\right)\right.}{2 C_{1} \lambda_{m+1}^{1 / 2} v N_{10}^{2}\left(\gamma, \sigma / 2, \sigma, N_{3}, v, f\right)} \\
& \times\left(4 C_{1} N_{3}+\gamma v C_{L}^{1 / 2} \lambda_{1}^{1 / 2}\right) \\
& +\frac{\lambda_{1}^{3 / 2}}{32 C_{1}^{2} v N_{10}^{2}\left(\gamma, \sigma / 2, \sigma, N_{3}, \nu, f\right)}\left(\lambda_{1}^{1 / 2}+2 N_{10}\left(\gamma, \frac{\sigma}{2}, \sigma, N_{3}, v, f\right)\right. \\
& \left.\left.\quad \times\left(2 C_{1} N_{3}+\gamma v C_{L}^{1 / 2} \lambda_{1}^{1 / 2}+\frac{2 C_{1} \lambda_{1}^{1 / 2} N_{4}^{2}\left(\gamma, N_{3}, \nu, f\right)}{\lambda_{m+1}^{1 / 2}}\right)\right)\right] .
\end{aligned}
$$

The estimate (4.53) follows after this estimate and (4.69).

Let

$$
T_{4}\left(\gamma, \sigma, N_{1}, \nu, f\right)=\left[\frac{N_{16}\left(\gamma, \sigma, N_{1}, \nu, f\right)}{C_{0} \lambda_{1} \eta\left(\gamma, \sigma, \sigma / 2, \nu, N_{3}\right)}\right],
$$

where $\eta\left(\gamma, \sigma, \sigma / 2, v, N_{3}\right)$ is as in (4.42). When $m \geq M_{\mathrm{NLG}}\left(\gamma, \sigma, N_{1}, v, f\right)$, it is clear that $\left\|w_{n}^{*}\right\| \leq \eta\left(\gamma, \sigma, \sigma / 2, \nu, N_{3}\right)$, so the second part of the theorem has been proved.

\section{Postprocessing the stable Galerkin steady states}

In previous sections we have provided an explicit numerical criterion for the stabilization of steady state solutions of the Navier-Stokes equations with a certain class of linear feedback control operators. In this section we use an efficient numerical 
method, the postprocessing Galerkin method, which was introduced in [38], [39], [40], [65], to improve the error estimates (3.47) and (4.57).

Here we consider only the Galerkin case. One can obtain similar results for the nonlinear Galerkin case. Suppose $u_{m}^{\infty}, m \geq M_{\mathrm{GLK}}$ is the stable steady state solution of the Galerkin equations. $u^{\infty}$ is the steady state solution of the closedloop Navier-Stokes equations which we obtained by Theorem 3.2 on page 55. By using the postprocessing technique, we are able to get the following result.

Theorem 5.1. We denote by $\Phi_{s}: H_{m} \rightarrow H_{m}^{\perp}$ the so called steady Approximate Inertial Manifold ([76], see also [30], [35]), which is defined implicitly by

$$
\begin{aligned}
\Phi_{s}\left(u_{m}\right)=(\nu A)^{-1} Q_{m}\left\{f-B\left(u_{m}+\Phi_{s}\left(u_{m}\right), u_{m}\right.\right. & \left.+\Phi_{s}\left(u_{m}\right)\right) \\
& \left.-\gamma L\left(u_{m}+\Phi_{s}\left(u_{m}\right)\right)\right\}
\end{aligned}
$$

for $u_{m} \in H_{m}$, provided $m$ is large enough (see [76]). We define $v_{m}^{\infty}$ implicitly by

$$
\begin{aligned}
v_{m}^{\infty}=-\left(\mathcal{A}_{m}\left(u_{m}^{\infty}\right)\right)^{-1} P_{m}\left[B\left(u_{m}^{\infty}, \Phi_{s}\left(u_{m}^{\infty}+v_{m}^{\infty}\right)\right)+B\left(\Phi_{s}\left(u_{m}^{\infty}+v_{m}^{\infty}\right), u_{m}^{\infty}\right)\right) \\
\left.+\gamma L \Phi_{s}\left(u_{m}^{\infty}+v_{m}^{\infty}\right)\right],
\end{aligned}
$$

and denote by

$$
u^{*}=u_{m}^{\infty}+v_{m}^{\infty}+\Phi_{s}\left(u_{m}^{\infty}+v_{m}^{\infty}\right)
$$

Then under the same assumptions as in Theorem 3.2 on page 55, we have

$$
\left|u^{\infty}-u^{*}\right| \leq \frac{K_{P P}\left(\gamma, \sigma, v, f, K_{1}\right)}{\lambda_{m+1}^{2}}\left[1+\frac{1}{2} \log \left(\frac{\lambda_{m+1}}{\lambda_{1}}\right)\right]^{1 / 2},
$$

where

$$
K_{P P}\left(\gamma, \sigma, \nu, f, K_{1}\right)=K_{P}\left(\gamma, \sigma, \nu, f, K_{1}\right)\left(1+\frac{K^{*}}{\lambda_{m+1}^{1 / 2}}\right),
$$

where $K^{*}$ and $K_{P}\left(\gamma, \sigma, v, f, K_{1}\right)$ are specified as in (5.11) and (5.13).

Proof. We rewrite $u^{\infty}=p_{m}^{\infty}+q_{m}^{\infty}$ with $p_{m}^{\infty} \in H_{m}$ and $q_{m}^{\infty} \in Q_{m}$. They satisfy the following equations

$$
\begin{aligned}
& v A p_{m}^{\infty}+P_{m}\left[B\left(p_{m}^{\infty}+q_{m}^{\infty}, p_{m}^{\infty}+q_{m}^{\infty}\right)+\gamma L\left(p_{m}^{\infty}+q_{m}^{\infty}\right)\right]=P_{m} f, \\
& v A q_{m}^{\infty}+Q_{m}\left[B\left(p_{m}^{\infty}+q_{m}^{\infty}, p_{m}^{\infty}+q_{m}^{\infty}\right)+\gamma L\left(p_{m}^{\infty}+q_{m}^{\infty}\right)=Q_{m} f,\right. \\
& v A u_{m}^{\infty}+P_{m}\left[B\left(u_{m}^{\infty}, u_{m}^{\infty}\right)+\gamma L u_{m}^{\infty}\right]=P_{m} f .
\end{aligned}
$$


Thus, we have

$$
\begin{aligned}
p_{m}^{\infty}-u_{m}^{\infty}=-\left(\mathcal{A}_{m}\left(u_{m}^{\infty}\right)\right)^{-1} P_{m}\left[B \left(p_{m}^{\infty}-\right.\right. & \left.u_{m}^{\infty}, p_{m}^{\infty}-u_{m}^{\infty}\right)+B\left(p_{m}^{\infty}, q_{m}^{\infty}\right) \\
& \left.+B\left(q_{m}^{\infty}, p_{m}^{\infty}\right)+B\left(q_{m}^{\infty}, q_{m}^{\infty}\right)+\gamma L q_{m}^{\infty}\right] .
\end{aligned}
$$

Therefore, we get

$$
\begin{aligned}
& p_{m}^{\infty}-u_{m}^{\infty}-v_{m}^{\infty} \\
&=-\left(\mathcal{A}_{m}\left(u_{m}^{\infty}\right)\right)^{-1} P_{m}\left[B\left(p_{m}^{\infty}-u_{m}^{\infty}, p_{m}^{\infty}-u_{m}^{\infty}\right)+B\left(p_{m}^{\infty}, q_{m}^{\infty}\right)\right. \\
& \quad+B\left(q_{m}^{\infty}, p_{m}^{\infty}\right)+B\left(q_{m}^{\infty}, q_{m}^{\infty}\right)+\gamma L q_{m}^{\infty}-B\left(u_{m}^{\infty}, \Phi_{s}\left(u_{m}^{\infty}+v_{m}^{\infty}\right)\right) \\
&\left.\quad-B\left(\Phi_{s}\left(u_{m}^{\infty}+v_{m}^{\infty}\right), u_{m}^{\infty}\right)-\gamma L \Phi_{s}\left(u_{m}^{\infty}+v_{m}^{\infty}\right)\right] .
\end{aligned}
$$

Notice that, by using a similar technique as in the proof of (3.56) and (3.57), for all $w_{1}, w_{2} \in H$, and $w_{3} \in H_{m}$, we obtain

$$
\left|\left(\left(\mathcal{A}_{m}\left(u_{m}^{\infty}\right)\right)^{-1} B\left(w_{1}, w_{2}\right), w_{3}\right)\right| \leq C_{1}\left|w_{1}\right|\left|w_{2}\right|\left|A^{1 / 2}\left(\mathcal{A}_{m}\left(u_{m}^{\infty}\right)\right)^{-1} w_{3}\right|_{L^{\infty}} .
$$

Now let us recall the following inequality, which holds in the two dimensional case, from [11]

$$
\|u\|_{L^{\infty}} \leq C_{2}\|u\|\left[1+\log \left(\frac{|A u|}{\lambda_{1}^{1 / 2}\|u\|}\right)\right]^{1 / 2} .
$$

Therefore,

$$
\begin{aligned}
& \left|A^{1 / 2}\left(\mathcal{A}_{m}\left(u_{m}^{\infty}\right)\right)^{-1} w_{3}\right|_{L^{\infty}} \\
& \quad \leq C_{2}\left|A\left(\mathcal{A}_{m}\left(u_{m}^{\infty}\right)\right)^{-1} w_{3}\right|\left[1+\log \left(\frac{\left|A^{3 / 2}\left(\mathcal{A}_{m}\left(u_{m}^{\infty}\right)\right)^{-1} w_{3}\right|}{\lambda_{1}^{1 / 2}\left|A\left(\mathcal{A}_{m}\left(u_{m}^{\infty}\right)\right)^{-1} w_{3}\right|}\right)\right]^{1 / 2} .
\end{aligned}
$$

By using (3.28) with $\lambda=0, \rho=\sigma, s=\sigma, R=K_{3}$, and the fact $w_{3} \in H_{m}$, we conclude

$$
\left|A^{1 / 2}\left(\mathcal{A}_{m}\left(u_{m}^{\infty}\right)\right)^{-1} w_{3}\right|_{L^{\infty}} \leq C_{2} K_{9}\left(\gamma, \sigma, \sigma, \nu, K_{3}\right)\left|w_{3}\right|\left[1+\log \left(\frac{\lambda_{m+1}^{1 / 2}}{\lambda_{1}^{1 / 2}}\right)\right]^{1 / 2} .
$$

Moreover,

$$
\begin{aligned}
& \left|\left(\mathcal{A}_{m}\left(u_{m}^{\infty}\right)\right)^{-1} B\left(w_{1}, w_{2}\right)\right| \\
& \quad \leq C_{1} C_{2} K_{9}\left(\gamma, \sigma, \sigma, v, K_{3}\right)\left[1+\frac{1}{2} \log \left(\frac{\lambda_{m+1}}{\lambda_{1}}\right)\right]^{1 / 2}\left|w_{1}\right|\left|w_{2}\right|
\end{aligned}
$$


As a result of the inequality above, we have

$$
\begin{aligned}
& \left|\left(\mathcal{A}_{m}\left(u_{m}^{\infty}\right)\right)^{-1} B\left(p_{m}^{\infty}-u_{m}^{\infty}, p_{m}^{\infty}-u_{m}^{\infty}\right)\right| \\
& \quad \leq C_{1} C_{2} K_{9}\left(\gamma, \sigma, \sigma, v, K_{3}\right)\left[1+\frac{1}{2} \log \left(\frac{\lambda_{m+1}}{\lambda_{1}}\right)\right]^{1 / 2}\left|p_{m}^{\infty}-u_{m}^{\infty}\right|^{2}, \\
& \left|\left(\mathcal{A}_{m}\left(u_{m}^{\infty}\right)\right)^{-1} B\left(p_{m}^{\infty}-u_{m}^{\infty}, q_{m}^{\infty}\right)\right| \\
& \quad \leq C_{1} C_{2} K_{9}\left(\gamma, \sigma, \sigma, v, K_{3}\right)\left[1+\frac{1}{2} \log \left(\frac{\lambda_{m+1}}{\lambda_{1}}\right)\right]^{1 / 2}\left|p_{m}^{\infty}-u_{m}^{\infty}\right|\left|q_{m}^{\infty}\right|, \\
& \left|\left(\mathcal{A}_{m}\left(u_{m}^{\infty}\right)\right)^{-1} B\left(q_{m}^{\infty}, p_{m}^{\infty}-u_{m}^{\infty}\right)\right| \\
& \quad \leq C_{1} C_{2} K_{9}\left(\gamma, \sigma, \sigma, v, K_{3}\right)\left[1+\frac{1}{2} \log \left(\frac{\lambda_{m+1}}{\lambda_{1}}\right)\right]^{1 / 2}\left|p_{m}^{\infty}-u_{m}^{\infty}\right|\left|q_{m}^{\infty}\right|, \\
& \quad\left|\left(\mathcal{A}_{m}\left(u_{m}^{\infty}\right)\right)^{-1} B\left(u_{m}^{\infty}, q_{m}^{\infty}-\Phi_{s}\left(u_{m}^{\infty}+v_{m}^{\infty}\right)\right)\right| \\
& \quad \leq C_{1} C_{2} K_{9}\left(\gamma, \sigma, \sigma, v, K_{3}\right)\left[1+\frac{1}{2} \log \left(\frac{\lambda_{m+1}}{\lambda_{1}}\right)\right]^{1 / 2}\left|u_{m}^{\infty}\right|\left|q_{m}^{\infty}-\Phi_{s}\left(u_{m}^{\infty}+v_{m}^{\infty}\right)\right|, \\
& \left|\left(\mathcal{A}_{m}\left(u_{m}^{\infty}\right)\right)^{-1} B\left(q_{m}^{\infty}-\Phi_{s}\left(u_{m}^{\infty}+v_{m}^{\infty}\right), u_{m}^{\infty}\right)\right| \\
& \quad \leq C_{1} C_{2} K_{9}\left(\gamma, \sigma, \sigma, v, K_{3}\right)\left[1+\frac{1}{2} \log \left(\frac{\lambda_{m+1}}{\lambda_{1}}\right)\right]^{1 / 2}\left|u_{m}^{\infty}\right|\left|q_{m}^{\infty}-\Phi_{s}\left(u_{m}^{\infty}+v_{m}^{\infty}\right)\right|, \\
& \left|\left(\mathcal{A}_{m}\left(u_{m}^{\infty}\right)\right)^{-1} B\left(q_{m}^{\infty}, q_{m}^{\infty}\right)\right| \\
& \quad \leq C_{1} C_{2} K_{9}\left(\gamma, \sigma, \sigma, v, K_{3}\right)\left[1+\frac{1}{2} \log \left(\frac{\lambda_{m+1}}{\lambda_{1}}\right)\right]^{1 / 2}\left|q_{m}^{\infty}\right|^{2}, \\
& \left|\left(\mathcal{A}_{m}\left(u_{m}^{\infty}\right)\right)^{-1} \gamma L\left(q_{m}^{\infty}-\Phi_{s}\left(u_{m}^{\infty}+v_{m}^{\infty}\right)\right)\right| \\
& \quad \leq \frac{\gamma v C_{L}^{1-\alpha} \lambda_{1}^{1-\alpha} \lambda_{m}^{1-\alpha}\left(\gamma, \sigma, \sigma, v, K_{3}\right)\left|q_{m}^{\infty}-\Phi_{s}\left(u_{m}^{\infty}+v_{m}^{\infty}\right)\right| .}{}
\end{aligned}
$$

Therefore,

$$
\begin{aligned}
& \left|p_{m}^{\infty}-u_{m}^{\infty}-v_{m}^{\infty}\right| \\
& \leq C_{1} C_{2} K_{9}\left(\gamma, \sigma, \sigma, v, K_{3}\right)\left[1+\frac{1}{2} \log \left(\frac{\lambda_{m+1}}{\lambda_{1}}\right)\right]^{1 / 2} \\
& \times\left[\left|p_{m}^{\infty}-u_{m}^{\infty}\right|^{2}+2\left|p_{m}^{\infty}-u_{m}^{\infty}\right|\left|q_{m}^{\infty}\right|+2\left|u_{m}^{\infty}\right|\left|q_{m}^{\infty}-\Phi_{s}\left(u_{m}^{\infty}+v_{m}^{\infty}\right)\right|+\left|q_{m}^{\infty}\right|^{2}\right] \\
& \quad+\frac{\gamma v C_{L}^{1-\alpha} \lambda_{1}^{1-\alpha}}{\lambda_{m+1}^{1-\alpha}} K_{9}\left(\gamma, \sigma, \sigma, v, K_{3}\right)\left|q_{m}^{\infty}-\Phi_{s}\left(u_{m}^{\infty}+v_{m}^{\infty}\right)\right| .
\end{aligned}
$$


Now we assume that $m$ is large enough such that

$$
\begin{gathered}
\left|A\left(p_{m}^{\infty}+q_{m}^{\infty}\right)\right| \leq \frac{3}{2} \lambda_{1}^{1 / 2} K_{3}, \\
\left|A\left(u_{m}^{\infty}+v_{m}^{\infty}+\Phi_{s}\left(u_{m}^{\infty}+v_{m}^{\infty}\right)\right)\right| \leq \frac{3}{2} \lambda_{1}^{1 / 2} K_{3}, \\
v-\frac{3 C_{2} K_{2}}{2 \lambda_{m+1}}\left[1+\frac{1}{2} \log \left(\frac{\lambda_{m+1}}{\lambda_{1}}\right)\right]^{1 / 2}-\frac{3 C_{1} K_{3}}{2 \lambda_{m+1}}-\frac{v \gamma C_{L}^{1-\alpha} \lambda_{1}^{1-\alpha}}{\lambda_{m+1}^{2-2 \alpha}} \geq \frac{v}{2} .
\end{gathered}
$$

Notice that all the above assumptions are true if $m$ is at least of order $G$, the Grashoff number. In fact, to implement the results of our previous sections we require $m$ to be much larger, namely, that $m \geq M_{\mathrm{GLK}}$.

Next we get an estimate for $\left|q_{m}^{\infty}-\Phi_{s}\left(u_{m}^{\infty}+v_{m}^{\infty}\right)\right|$. Denote by $w_{m}=p_{m}^{\infty}-$ $u_{m}^{\infty}-v_{m}^{\infty}$ and by $w_{m}^{\perp}=q_{m}^{\infty}-\Phi_{s}\left(u_{m}^{\infty}+v_{m}^{\infty}\right)$. Following the equations (5.1) and (5.5), we obtain

$$
\begin{aligned}
v A w_{m}^{\perp}+Q_{m} & {\left[B\left(p_{m}^{\infty}+q_{m}^{\infty}, w_{m}+w_{m}^{\perp}\right)\right.} \\
+ & B\left(w_{m}+w_{m}^{\perp}, u_{m}^{\infty}+v_{m}^{\infty}+\Phi_{s}\left(u_{m}^{\infty}+v_{m}^{\infty}\right)\right)+\gamma L\left(w_{m}+w_{m}^{\perp}\right)=0 .
\end{aligned}
$$

Following the inequalities (2.7)-(2.11) and (5.7), and (2.20) we get

$$
\begin{gathered}
\left(v-\frac{3 C_{2} K_{2}}{2 \lambda_{m+1}}\left[1+\frac{1}{2} \log \left(\frac{\lambda_{m+1}}{\lambda_{1}}\right)\right]^{1 / 2}-\frac{3 C_{1} K_{3}}{2 \lambda_{m+1}}-\frac{v \gamma C_{L}^{1-\alpha} \lambda_{1}^{1-\alpha}}{\lambda_{m+1}^{2-2 \alpha}}\right)\left\|w_{m}^{\perp}\right\| \\
\leq \frac{C_{1} C_{2}\left\|p_{m}^{\infty}\right\|\left|w_{m}\right|}{\lambda_{m+1}^{1 / 2}}\left[1+\frac{1}{2} \log \left(\frac{\lambda_{m+1}}{\lambda_{1}}\right)\right]^{1 / 2} \\
+C_{1}\left|w_{m}\right|^{1 / 2}\left\|w_{m}\right\|^{1 / 2}\left|q_{m}^{\infty}\right|^{1 / 2}\left\|q_{m}^{\infty}\right\|^{1 / 2}+C_{1}\left|u_{m}^{\infty}\right|^{1 / 2}\left|A u_{m}^{\infty}\right|^{1 / 2}\left|w_{m}\right| \\
+C_{1}\left|w_{m}\right|^{1 / 2}\left\|w_{m}\right\|^{1 / 2}\left|\Phi_{s}\left(u_{m}^{\infty}+v_{m}^{\infty}\right)\right|^{1 / 2}\left\|\Phi_{s}\left(u_{m}^{\infty}+v_{m}^{\infty}\right)\right\|^{1 / 2} \\
+\frac{\gamma \nu C_{L}^{1-\alpha} \lambda_{1}^{1-\alpha}}{\lambda_{m+1}^{1 / 2-\alpha}}|w| .
\end{gathered}
$$

Therefore, by using the assumptions (5.8) and (5.9) we conclude

$$
\left|w_{m}^{\perp}\right| \leq \frac{K^{*}}{\lambda_{m+1}^{1 / 2}}\left|w_{m}\right|
$$

where 
$(5.11)$

$$
\begin{aligned}
K^{*}=\frac{2}{v}\left[\frac { 3 C _ { 1 } C _ { 2 } \lambda _ { 1 } ^ { 1 / 2 } K _ { 3 } } { 2 \lambda _ { m + 1 } ^ { 1 / 2 } } \left[1+\frac{1}{2}\right.\right. & \left.\log \left(\frac{\lambda_{m+1}}{\lambda_{1}}\right)\right]^{1 / 2} \\
& \left.+\frac{3 C_{1} \lambda_{1}^{1 / 2} K_{3}}{\lambda_{m+1}^{1 / 2}}+C_{1} K_{3}+\frac{\gamma \nu C_{L}^{1-\alpha} \lambda_{1}^{1-\alpha}}{\lambda_{m+1}^{1 / 2-\alpha}}\right] .
\end{aligned}
$$

Thus, if $m$ is large enough such that

$$
\begin{aligned}
& K^{*} K_{9}\left(\gamma, \sigma, \sigma, v, K_{3}\right) \\
& \quad \times\left[\frac{2 C_{1} C_{2} K_{1}}{\lambda_{m+1}}\left[1+\frac{1}{2} \log \left(\frac{\lambda_{m+1}}{\lambda_{1}}\right)\right]^{1 / 2}+\frac{\gamma \nu C_{L}^{1-\alpha} \lambda_{1}^{1-\alpha}}{\lambda_{m+1}^{3 / 2-\alpha}}\right] \leq \frac{1}{2},
\end{aligned}
$$

we get

$$
\left|p_{m}^{\infty}-u_{m}^{\infty}-v_{m}^{\infty}\right| \leq \frac{K_{P}\left(\gamma, \sigma, v, f, K_{1}\right)}{\lambda_{m+1}^{2}}\left[1+\frac{1}{2} \log \left(\frac{\lambda_{m+1}}{\lambda_{1}}\right)\right]^{1 / 2},
$$

where

$$
\begin{aligned}
K_{P}\left(\gamma, \sigma, \nu, f, K_{1}\right)=2 C_{1} C_{2} K_{9}\left(\gamma, \sigma, \sigma, v, K_{3}\right) & \\
& \times\left(K_{10}\left(\gamma, \sigma, \sigma, \nu, K_{3}\right)+\frac{3}{2} K_{3}\right)^{2} .
\end{aligned}
$$

Notice that, when $m \geq M_{\mathrm{GLK}}$, (5.12) is true. Moreover, we have

$$
\left|q_{m}^{\infty}-\Phi_{s}\left(u_{m}^{\infty}\right)\right| \leq \frac{K^{*} K_{P}\left(\gamma, \sigma, v, f, K_{1}\right)}{\lambda_{m+1}^{5 / 2}}\left[1+\frac{1}{2} \log \left(\frac{\lambda_{m+1}}{\lambda_{1}}\right)\right]^{1 / 2} .
$$

Then the estimate (5.2) follows.

\section{CONCLUSION}

In this paper we have presented an explicit numerical criterion for the stabilization of steady state solutions of the Navier-Stokes equations with linear feedback control. We will summarize below our results and the steps of how to implement this criterion.

Suppose $u^{*}$ is an unstable steady state solution of the NSE. It is not practical, computationally, to stabilize the infinite dimensional system. However, by using numerical methods we can find a stationary solution of the Galerkin approximation equations, or the nonlinear Galerkin approximation equations, which is an approximation of $u^{*}$. Suppose that we can design a linear feedback control $L$, whose Galerkin (or nonlinear Galerkin) truncation stabilizes this approximate solution. The question is whether this same linear feedback control, $L$, stabilizes 
$u^{*}$. In this paper we have shown that the answer to this question is affirmative, provided we use enough eigenmodes, and the linear feedback operator $L$ belongs to a certain class of linear operators which are dominated by the viscous term $v A$. This restriction on the feedback controllers is essential in order for the viscous term to suppress the potential spillover that $L$ might cause, by destabilizing the higher eigenmodes. This approach is practical because we are now working with a finite dimensional system.

A general abstract algorithm. Our general algorithm is as follows:

(i) Find a steady state $u_{m}^{*}$ of the $m$-state Galerkin approximation equations, or a steady state $p_{m}^{*}+q_{m}^{*}$ of the $m$-state nonlinear Galerkin approximation equations, by numerical simulation for some $m$, such that $u_{m}^{*}$, or $p_{m}^{*}+q_{m}^{*}$, is close to $u^{*}$, respectively. More precisely, we assume that, in the Galerkin case,

$$
\left|u^{*}-u_{m}^{*}\right| \leq \varepsilon \ll 1,
$$

or in the nonlinear Galerkin case,

$$
\left|u^{*}-\left(p_{m}^{*}+q_{m}^{*}\right)\right| \leq \varepsilon \ll 1 .
$$

(ii) Design a linear feedback controller $L$, which might act on finite or infinitely many modes, and which belongs to the class of linear operators described in Section 2, such that $u_{m}^{*}$ is a stable stationary solution of the closed-loop system

$$
\frac{d u_{m}}{d t}+v A u_{m}+P_{m} B\left(u_{m}, u_{m}\right)+P_{m} L\left(u_{m}-u_{m}^{*}\right)=P_{m} g
$$

in the Galerkin case, or $p_{m}^{*}+q_{m}^{*}$ is a stable stationary solution of the following closed-loop system

$$
\begin{aligned}
\frac{d p_{m}}{d t}+v A p_{m}+P_{m} B\left(p_{m}\right. & \left.+q_{m}, p_{m}+q\right)-P_{m} B\left(q_{m}, q_{m}\right) \\
& +P_{m} L\left(p_{m}+q_{m}-p_{m}^{*}-q_{m}^{*}\right)=P_{m} \mathcal{g}, \\
v A q_{m}+Q_{m} B\left(p_{m}, p_{m}\right) & +Q_{m} L\left(p_{m}-p_{m}^{*}\right)=Q_{m} g
\end{aligned}
$$

in the nonlinear Galerkin case.

(iii) Let $\sigma>0$ be the lower bound of the real part of eigenvalues of the linearized operator, $\mathcal{A}_{m}\left(u_{m}^{*}\right)$ in the Galerkin case, or $\mathcal{N}_{m}\left(p_{m}^{*}\right)$ in the nonlinear Galerkin case $\left(\mathcal{A}_{m}\left(u_{m}^{*}\right)\right.$ and $\mathcal{N}_{m}\left(p_{m}^{*}\right)$ are defined in Sections 3 on page 44 and 4 on page 60 , respectively). 
(iv) Let $M_{\mathrm{GLK}}$ and $M_{\mathrm{NLG}}$ be as in Section 3 and Section 4, respectively. If $m \geq$ $M_{\mathrm{GLK}}$ in the Galerkin case, or $m \geq M_{\mathrm{NLG}}$ in the nonlinear Galerkin case, then the feedback controller $L$ will stabilize a steady state solution $u^{* *}$ of the closed-loop Navier-Stokes equations

$$
\frac{d u}{d t}+v A u+B(u, u)+L\left(u-u_{m}^{*}\right)=g .
$$

Moreover, one has the following error estimate for the Galerkin case

$$
\left|u^{* *}-u_{m}^{*}\right| \leq \frac{C}{\lambda_{m+1}} \quad \text { and }\left|u^{* *}-u^{*}\right| \leq \frac{C}{\lambda_{m+1}}+\varepsilon,
$$

or in the nonlinear Galerkin case,

$$
\left|u^{* *}-p_{m}^{*}-q_{m}^{*}\right| \leq \frac{C}{\lambda_{m+1}^{3 / 2}} \quad \text { and }\left|u^{* *}-u^{*}\right| \leq \frac{C}{\lambda_{m+1}^{3 / 2}}+\varepsilon .
$$

Furthermore, one can use the postprocessing procedure $[38,39,65]$ to improve the accuracy of both (Galerkin as well as nonlinear Galerkin) methods as it has been discussed in Section 5 on page 83. As a result the above error estimates, one may conclude that $u^{* *}$ is very close to the original solution $u^{*}$, provided $m$ is large enough, and that $p_{m}^{*}+q_{m}^{*}$ converges to $u^{* *}$ faster than $u^{*}$ does.

(v) Otherwise, if either step 3 or step 4 does not hold we either design another linear feedback control that allows for a larger $\sigma$, or increase $m$ and repeat the above procedure.

In this way one is guaranteed that the linear feedback control $L$ for the finite system ODEs will stabilize a steady state solution $u^{* *}$ of the closed-loop NSE, and $u^{* *}$ is nearby the unstable steady state $u^{*}$ of the NSE.

A specific implementation of the algorithm. Next, we present a special case of the above general algorithm, which will guarantee an a priori upper estimate for $\varepsilon$, given in step 1 of the above general algorithm, of order $O\left(\lambda_{m+1}^{-1}\right)$ in the Galerkin case, and of order $O\left(\lambda_{m+1}^{-3 / 2}\right)$ in the nonlinear Galerkin case.

Let $u^{*}$ be, as before, an unstable steady state of the NSE (open-loop)

$$
\frac{d u}{d t}+v A u+B(u, u)=g,
$$

which we are interested in stabilizing. Set $u^{*}=\xi_{m}^{*}+\eta_{m}^{*}$, where $\xi_{m}^{*}=P_{m} u^{*}$ and $\eta_{m}^{*}=\left(I-P_{m}\right) u^{*}$. It is clear that $u_{m}=\xi_{m}^{*}$ is a steady state of the following Galerkin system

$$
\begin{aligned}
\frac{d u_{m}}{d t}+v A u_{m} & +P_{m} B\left(u_{m}, u_{m}\right)+\gamma P_{m} L u_{m} \\
& =P_{m}\left[g+B\left(\xi_{m}^{*}, \xi_{m}^{*}\right)-B\left(u^{*}, u^{*}\right)+\gamma L \xi_{m}^{*}\right] .
\end{aligned}
$$


The equation above is the Galerkin procedure at level $m$ of the closed-loop system:

$$
\frac{d u}{d t}+v A u+B(u, u)+\gamma L u=f
$$

where $f=g+B\left(\xi_{m}^{*}, \xi_{m}^{*}\right)-B\left(u^{*}, u^{*}\right)+\gamma L \xi_{m}^{*}$.

Notice that the above equation is within our frame of work. In order for us to derive explicit values to the constants $M_{\mathrm{GLK}}$ and $M_{\mathrm{NLG}}$ we would need to provide an explicit bound for $\|f\|$ (see the end of Section 3.1 on page 48). Indeed, it is sufficient to estimate the right hand side of the following

$$
\|f\| \leq\|g\|+\left\|B\left(\xi_{m}^{*}, \xi_{m}^{*}\right)\right\|+\left\|B\left(u^{*}, u^{*}\right)\right\|+\left\|\gamma L \xi_{m}^{*}\right\| .
$$

First, notice that by (2.11)

$$
\left\|B\left(u^{*}, u^{*}\right)\right\| \leq 2 C_{1}\left|u^{*}\right|^{1 / 2}\left|A u^{*}\right|^{3 / 2},
$$

and

$$
\left\|B\left(\xi_{m}^{*}, \xi_{m}^{*}\right)\right\| \leq 2 C_{1}\left|\xi_{m}^{*}\right|^{1 / 2}\left|A \xi_{m}^{*}\right|^{3 / 2} \leq 2 C_{1}\left|u^{*}\right|^{1 / 2}\left|A u^{*}\right|^{3 / 2}
$$

Based on part (ii) of Lemma 3.1 on page 44 we have

$$
\left|u^{*}\right| \leq \frac{|g|}{\lambda_{1} v} \quad \text { and } \quad\left|A u^{*}\right| \leq K(|g|)=\frac{2|g|}{v}+\frac{C_{1}^{2}|g|^{3}}{\lambda_{1}^{2} v^{5}}
$$

Thus, we obtain

$$
\left\|B\left(u^{*}, u^{*}\right)\right\|+\left\|B\left(\xi_{m}^{*}, \xi_{m}^{*}\right)\right\| \leq \widetilde{K}(|g|),
$$

with $\tilde{K}(|g|)=\left[4 C_{1} K^{3 / 2}(|g|)|g|\right] /\left(\lambda_{1} v\right)$.

As in Section 2 on page 41, we assume that (2.20) holds for $\alpha=0$ and $\beta=\frac{1}{2}$. Therefore, we have

$$
\gamma\left\|L \xi_{m}^{*}\right\| \leq v \lambda_{1} C_{L}\left|A^{1 / 2} \xi_{m}^{*}\right| \leq v \lambda_{1} C_{L}\left|A^{1 / 2} u^{*}\right| \leq \lambda_{1}^{1 / 2} C_{L}|g| .
$$

From the above we conclude that

$$
\|f\| \leq \bar{K}\left(|g|,\|g\|, \gamma, C_{L}\right)=\lambda_{1}^{1 / 2} C_{L}|g|+\widetilde{K}(|g|)+\|g\| .
$$

One can restrict further the class of linear feedback operator $L$ by imposing an upper bound on $C_{L}$, and hence give an explicit a priori determined upper bound to $\|f\|$. 
Now, we return to apply the general algorithm under these specific choices for $u_{m}^{*}$ and $f$. Indeed, in step 1 of the general algorithm we have $u_{m}^{*}=\xi_{m}^{*}$ and therefore

$$
\left|u^{*}-u_{m}^{*}\right|=\left|\eta_{m}^{*}\right| \leq \frac{1}{\lambda_{m+1}}\left|A \eta_{m}^{*}\right| \leq \frac{1}{\lambda_{m+1}}\left|A u^{*}\right| \leq \frac{K(|g|)}{\lambda_{m+1}} .
$$

Then we take $\varepsilon=K(|g|) / \lambda_{m+1}$, which is very small provided $m$ is large enough. Following the same steps as in the general algorithm for this choice of $f$ and $u_{m}^{*}$, we obtain in step 4 that

$$
\left|u^{*}-u^{* *}\right| \leq \frac{C}{\lambda_{m+1}}+\frac{K(|g|)}{\lambda_{m+1}} .
$$

Hence, the stable steady state solution $u^{* *}$ of the closed-loop is not far away from the original unstable steady state of the open-loop $u^{*}$.

We can design a similar algorithm for the nonlinear Galerkin case with a new specific $f$ which satisfies the right estimates so that

$$
\varepsilon=O\left(\lambda_{m+1}^{-3 / 2}\right)
$$

in the nonlinear Galerkin case, and hence step 4 in this situation would lead to

$$
\left|u^{*}-u^{* *}\right|=O\left(\lambda_{m+1}^{-3 / 2}\right)
$$

Acknowledgements. This work was supported in part by the National Science Foundation - Division of Mathematical Sciences. CC and EST are grateful for the warm hospitality of the IGPP, Los Alamos National Laboratory, where this work was initiated. EST also acknowledges the Varon Fellowship at the Weizmann Institute, and IGK the Alexander von Humboldt Foundation at the Fritz-HaberInstitut, where the work was completed. Discussions with Dr. S. Shvartsman are gratefully acknowledged.

\section{REFERENCES}

[1] F. Abergel \& R. Temam, On some control problems in fluid mechanics, Theor. Comput. Fluid Dyn. 1 (1990), 303-325.

[2] R.A. Adams, Sobolev Spaces, Academic Press, New York, 1975.

[3] M.J. BALAS, Feedback control of linear diffusion processes, Int. J. Control 29 (1979), 523-533.

[4] - Reduced-order feedback control of distributed parameter systems via singular-perturbation methods, Jour. Math. Anal. Appl. 87 (1982), 281-294.

[5] _ . Toward a more practical control theory of linear distributed parameter systems, Control and Dynamic Systems: Advances in Theory and Appl. (C.T. Leondes, eds.), Volume 18, (1982), 361-421.

[6] The Galerkin method and feedback control of linear distributed parameter systems, Jour. Math. Anal. Appl. 91 (1983), 526-546. 
[7] _ Nonlinear finite-dimensional control of a class of nonlinear distributed parameter systems using residual-mode filters: a proof of local exponential stability, Jour. Math. Anal. Appl. 162 (1991), 63-70.

[8] B.T. BAnSENAUER \& M.J. BALAS, Reduced order model based control of the flexible articulatedtruss space crane, Journ. Guid. Cont. Dyn. 18 (1995), 135-142.

[9] V. BARBU \& S.S. SRITHARAN, $H^{\infty}$-control theory of fluid dynamics, Proc. Roy. Soc. London 454 (1998), 3009-3033.

[10] D.L. BOLEY, Krylov space methods on state-space control models, Circ. Syst. Signal Proc. 13 (1994), 733-758.

[11] H. BrÉZIS, \& T. Gallouet, Nonlinear Schrödinger evolution equations, Nonlinear Anal. TMA 4 (1980), 677-681.

[12] P. BRUNOVSKY, Controlling the dynamics of scalar reaction diffusion equations by finite dimensional controllers, In: Modeling and Inverse Problems of Control for Distributed Parameter Systems (A. Kurzhanski \& I. Lasiecka, eds.), 1991.

[13] J.A. BURNS \& Y.-R. OU, Feedback control of the driven cavity problem using LQR designs, Proceedings of the 33rd CDC, 289-294, 1994.

[14] H. CHOI, R. TEMAM, P. MOIN \& J. KIM, Feedback control for unsteady flow and its application to the stochastic Burgers equation, J. Fluid Mech. 253 (1993), 509-543.

[15] P.D. Christofides \& P. DAOUTIDIS, Finite-dimensional control of parabolic PDE systems using approximate inertial manifolds, Jour. Math. Anal. Appl. 216 (1997), 398-420.

[16] B. COCKBURN, D.A. JONES \& E.S. TITI, Estimating the number of asymptotic degrees of freedom for nonlinear dissipative systems, Math. Comp. 66 (1997), 1073-1087.

[17] P. CONSTANTIN \& C. Foias, Navier-Stokes Equations, The University of Chicago Press, 1988.

[18] P. Constantin, C. Foias, B. Nicolaenko \& R. Temam, Integral Manifolds and Inertial Manifolds for Dissipative Partial Differential Equations, Applied Mathematics Sciences 70, Springer-Verlag, 1988.

[19] _ Spectral barriers and integral manifolds for dissipative partial differential equations, J. Dynamics Differential Equations 1 (1989), 45-73.

[20] P. COnSTANTIN, C. FOIAS \& R. TEMAM, On the large time Galerkin approximation of the Navier-Stokes Equations, SIAM J. Numer. Anal. 21 (1984), 615-634.

[21] $\longrightarrow$ On the dimension of the attractors in two-dimensional turbulence, Phys. D 30 (1988), 284-296.

[22] R.F. CURTAIn, Pole assignment for distributed systems by finite-dimensional control, Automatica 21 (1985), 57-67.

[23] C. DeVulder, M. MARIOn \& E.S. Titi, On the rate of convergence of the nonlinear Galerkin methods, Math. Comp. 60 (1993), 495-514.

[24] N. Dunford \& J.T. SChWARTZ, Linear Operators, Part II: Spectral Theory Wiley Classics Library Edition, 1988.

[25] M.A. ERICKSON \& A.J. LAUB, An algorithmic test for checking stability of feedback spectral systems, Automatica 31 (1995), 125-135.

[26] C. Foias, O.P. Manley \& R. Temam, Modelling of the interaction of small and large eddies in two dimensional turbulent flows, RAIRO Modél. Math. Anal. Numér. 22 (1988), 93-114.

[27] _ Approximate inertial manifolds and effective viscosity in turbulent flows, Phys. Fluids A 3 (1991), 898-911.

[28] C. Foias, B. Nicolaenko, G.R. Sell \& R. Temam, Inertial manifolds for the KuramotoSivashinsky equation and an estimate of their lowest dimensions, J. Math. Pures Appl. 67 (1988), 197-226.

[29] C. FOIAS \& G. PRODI, Sur le comportement global des solutions non stationnaires des équations de Navier-Stokes en dimension deux, Rend. Sem. Mat. Univ. Padova, 39 (1967), 1-34.

[30] C. FOIAS \& J.C. SAUT, Remarques sur les équations de Navier-Stokes stationnaires, Ann. Scuola Norm. Sup. Pisa Cl. Sci. 10 (1983), 169-177. 
[31] C. Foias, G.R. Sell \& R. Temam, Variétés inertielles des équations différentielles dissipatives, C. R. Acad. Sci. Paris, Sér. I 301 (1985), 139-142.

[32] _ Inertial manifolds for nonlinear evolutionary equations, J. Differential Equations 73 (1988), 309-335.

[33] C. FOIAS, G.R. SELL \& E.S. TITI, Exponential tracking and approximation of inertial manifolds for dissipative nonlinear equations, J. Dynamics Differential Equations 1 (1989), 199-243.

[34] C. FOIAS \& R. TEMAM, Structure of the set of stationary solutions of the Navier-Stokes equations, Comm. Pure Appl. Math. 30 (1977), 149-164.

[35] _ Remarques sur les équations de Navier-Stokes stationaires et les phénoménes successifs de bifurcation, Analli Scu. norm. sup. Pisa 5 (1978), 29-63.

[36] M. GAD-EL-HaK, Flow Control: Fundamentals and Practices, (A. Pollard \& J.-P. Bonnet, eds.), Springer-Verlag, 1998.

[37] G.P. GALDI, An Introduction to the Mathematical Theory of the Navier-Stokes Equations, Vol. I \& II, Springer-Verlag, 1994.

[38] B. García-Archilla, J. Novo \& E.S. Titi, Postprocessing the Galerkin method: a novel approach to approximate inertial manifolds, SIAM J. Num. Anal. 35 (1998), 941-972.

[39] - An approximate inertial manifolds approach to postprocessing the Galerkin method for the Navier-Stokes equations, Math. Comp. 68 (1999), 893-911.

[40] B. García-ARChilla \& E.S. TiTI, Postprocessing the Galerkin method: the finite element case, SIAM J. Num. Anal., to appear.

[41] M.D. GUNZBURGER, L. Hou \& T. SVOBODNY, Boundary velocity control of incompressible flow with application to viscous drag reduction, SIAM J. Control Opt. 30 (1992), 167-181.

[42] J.K. HALE, Asymptotic Behavior of Dissipative Systems, AMS Mathematical Surveys and Monographs 25, Rhode Island, 1988.

[43] P. Holmes, J.L. Lumley \& G. Berkooz, Turbulence, Coherent Structures, Dynamical Systems and Symmetry, Cambridge University Press, 1996.

[44] J.T. HSU \& L. VU-QUOC, A rational formulation of thermal circuit models for electrothermal simulation - Part 2: Model reduction techniques, IEEE Trans. Circ. Syst. 143 (1996), 733-744.

[45] A. ISIDORI, Nonlinear Control Systems, Springer-Verlag, 1995.

[46] M.S. Jolly, I.G. KeVReKIDIS \& E.S. TiTi, Approximate inertial manifolds for the KuramotoSivashinsky equation: analysis and computations, Physica, D 44 (1990), 38-60.

[47] __ Preserving dissipation in approximate inertial forms for the Kuramoto-Sivashinsky equation, J. Dynamics Differential Equations 3 (1991), 179-197.

[48] D. JONES, L. MARgOLIN \& E.S. TITI, On the effectiveness of the approximate inertial manifolds - computational study, Theoretical and Computational Fluid Dynamics, 7 (1995), 243-260.

[49] D.A. JONES \& A.M. STUART, Attractive invariant manifolds under approximation: inertial manifolds, J. Diff. Eq. 123 (1995), 588-637.

[50] D.A. JONES, A.M. STUART \& E.S. TITI, Persistence of invariant sets for partial differential equations, Jour. Math. Anal. Appl. 219 (1998), 479-502.

[51] D.A. JONES \& E.S. TITI, Upper bounds on the number of determining modes, nodes, and volume elements for the Navier-Stokes equations, Indiana Univ. Math. J. 42 (1993), 875-887.

[52] __ A remark on quasi-stationary approximate inertial manifolds for the Navier-Stokes equations, SIAM J. Math. Anal. 25 (1994), 894-914.

[53] _ $C^{1}$ approximations of inertial manifolds for dissipative nonlinear equations, J. Differential Equations 127 (1996), 54-86.

[54] D. JosePH, Stability of Fluid Motions, Springer-Verlag, 1976.

[55] S.S. JOSHI, J.L. SPEYER \& J. KIM, A systems theory approach to the feedback stabilization of infinitesimal and finite-amplitude disturbances in plane Poiseuille flow, J. Fluid Mech. 332 (1997), 157-184.

[56] M. KRstic, T. BeWley \& B. BAmieh, organizers: National Science Foundation Workshop on Control of Flows, http://www-mae.ucsd.edu/research/krstic/workshop.html, accessed September 17, 1999. 
[57] P.V. Kokotovic, H.K. Khalil \& J. O’Reilly, Singular Perturbation Methods in Control: Analysis and Design, Academic Press, 1986.

[58] O.A LadyZhenSKaya, Mathematical Theory of Viscous Incompressible Flow, Gordon and Breach, New York, English translation, 2nd ed., 1969.

[59] L.D. Landau \& E.M. Lifshitz, Fluid Mechanics, Pergamon, London, 1959.

[60] E. LIEB \& W. THIRRING, Inequalities for the moments for the eigenvalues of Schrödinger equations and their relation to Sobolev inequalities, Studies in Math. Phys.: Essays in Honor of Valentine Bargman, Princeton Univ. Press, Princeton, N. J., 1976, pp. 269-303.

[61] J. MALLET-PARET \& G. SELL, Inertial manifolds for reaction diffusion equations in higher space dimensions, J. Amer. Math. Soc. 1 (1988), 805-866.

[62] C. Marchioro \& V. MoAuro, A remark on the stability problem in fluid dynamics, Jour. Math. Anal. Appl. 128 (1987), 413-418.

[63] M. MARION \& R. TEMAM, Nonlinear Galerkin methods, SIAM J. Num. Anal. 26 (1989), $1139-1157$.

[64] G. MÉTIVIER, Valeurs propres d'opérateurs définis par la restriction de systèmes variationnels à des sous-espaces, J. Math. Pures Appl. 57 (1978), 133-156.

[65] J. Novo, E.S. TITI \& S. WYNNE, Efficient methods using high accuracy approximate inertial manifolds, submitted.

[66] V.A. Pliss \& G.R. SELL, Perturbations of attractors of differential equations, J. Differential Eq. 92 (1991), 100-124.

[67] G. PRODI, Teoremi di tipo local per il sistema di Navier-Stokes e stabilità delle soluzioni stazionarie, Rend. Sem. Mat. Univ. Padova 32 (1962), 374-397.

[68] Y. SAKAWA, Feedback stabilization of linear diffusion systems, SIAM J. Cont. Optim. 21 (1983), 667-676.

[69] H. SANO \& N. KUNIMATSU, Feedback control of semilinear diffusion systems: inertial manifolds for closed-loop systems, IMA Journal of Math. Control \& Information 11 (1994), 75-92.

[70] S. ShVARTSMAN \& I.G. KeVREKIDIS, Nonlinear model reduction for control of distributed systems: a computer-assisted study, AIChE J. 44 (1998), 1579-1595.

[71] T. SU \& R.R. CRAIG JR., Model reduction and control of flexible structures using Krylov vectors, Journal of Guidance Control and Dynamics 14 (1991), 260-267.

[72] R. TEMAM, Navier-Stokes equations and nonlinear functional analysis, CBMS Regional Conference in Applied Mathematics 41, SIAM, Philadelphia, 1983.

[73] _ Navier-Stokes Equations, Theory and Numerical Analysis, North-Holland, 1984.

[74] _ Infinite Dimensional Dynamical Systems in Mechanics and Physics, Appl. Math. Sci Volume 68, Springer, 1988.

[75] E.S. TITI, On a criterion for locating stable stationary solutions to the Navier-Stokes Equations, Nonlinear Anal. TMA 11 (1987), 1085-1102.

[76] - On approximate inertial manifolds to the Navier-Stokes Equations, Jour. Math. Anal. Appl. 149 (1990), 540-557.

ChOngSheng CAO:

Department of Mathematics

University of California

Irvine, CA 92697-3875, U. S. A.

Current address: Center for Nonlinear Studies, MS - 258

Los Alamos National Laboratory

Los Alamos, NM 87545, U. S. A.

E-MAIL: ccao@math.uci.edu, ccao@cnls.lanl.gov 
96 Chongsheng CaO, IoAnnis G. Kevrekidis \& Edriss S. Titi

IOANNIS G. KEVREKIDIS:

Department of Chemical Engineering

Princeton University

Princeton, New Jersey 08544, U. S. A.

E-MAIL: yannis@arnold.princeton.edu

EDRISS S. TITI:

Department of Mathematics, and

Department of Mechanical and Aerospace Engineering

University of California

Irvine, CA 92697-3875, U. S. A.

E-MAIL: etiti@math.uci.edu

Received: September 15-17, 2000. 